\title{
7 Miniaturized Alternatives to Conventional Sample Preparation Techniques for Solid Samples
}

\author{
Noelia Cabaleiro and Inmaculada de la Calle* \\ Department of Analytical and Food Chemistry, Faculty of Chemistry, University of Vigo, \\ Campus As Lagoas-Marcosende s/n, 36310 Vigo, Spain \\ *e-mail: incalle@uvigo.es
}

\subsection{Introduction}

In recent years, several efforts were made in relation to the miniaturization of conventional sample preparation procedures. Analytical chemistry researchers are concerned about the use of conventional protocols, which are generally large-scale, tedious, time-consuming, require manual labor and involve the use of large quantities of hazardous reagents. Thus, the development of new miniaturized procedures has become necessary due to the increasing demand for environmentally friendly, green, fast and alternative approaches.

Today, there is continuous research focused on improving sample preparation procedures. Miniaturization is one of the main trends in analytical chemistry in addition to simplification and automation (Valcárcel \& Cárcenas, 2000; Ríos et al., 2009). The main principles of miniaturization approaches focus on downsizing the methods by reduction of the amount of sample, reduction or elimination of chemical reagents and the development of one-step treatments, on-line procedures and in-thefield analysis. It is also desirable to design automated and unattended procedures (Halls, 1995; Ramos et al., 2005). Figure 7.1 shows the number of publications per year devoted to miniaturization of sample preparation procedures. As can be seen, there is a marked increase since 2000.

A special challenge regarding miniaturization is the analysis of solid samples, which often require advanced sample pre-treatment in order to be suitable for a specific type of analysis. Different instrumental techniques are applied for the analysis of organic and inorganic analytes and each presents unique challenges.

One option for the analysis of metal ions in solid samples is the direct solid analysis (Bendicho \& de Loos-Vollebregt, 1991; Stoeppler, 1997; Potts \& Robinson, 2003). However, sample pre-treatment may be necessary prior to the analysis with some instruments, if the solid sample is not the appropriate form. The limitations of direct solid analysis include: (i) reduced representativeness of subsamples; (ii) lack of appropriate calibration standards; (iii) time-consuming steps for multielemental analysis; (iv) high number of interferences; (v) difficult sample introduction (non automated) and (vi) low precision (Hoenig \& de Kersabiec, 1996; Ebdon et al., 2003). Due to these limitations, sample preparation of solids has received tremendous research attention during the last few years. 


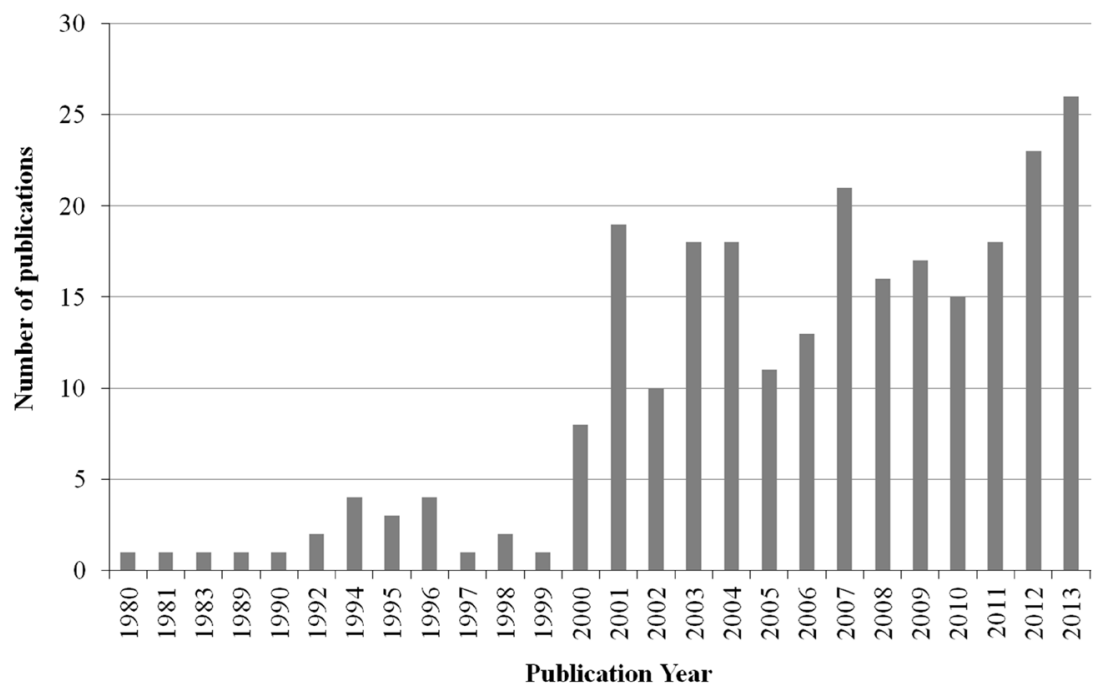

Figure 7.1 Number of publications devoted to miniaturized sample preparation procedures since 1980 (source: ISI Web of knowledge (Web of Science)-Thomson Reuters).

In this chapter, examples of conventional or established procedures for solid sample preparation and the miniaturized alternative approaches evolved from the previous protocols will be discussed. A scheme of the evolution from conventional sample preparation procedures to miniaturized versions can be seen in Figure 7.2. Moreover, a detailed description of the year of appearance of some conventional and miniaturized procedures is presented in Figure 7.3.

Conventional or established sample preparation procedures encompass the commonly applied procedures and reference methods used for validation purposes. These classical procedures involve several approaches that can be classified as: procedures involving no sample decomposition (slurry sampling, SS), procedures involving sample decomposition (such as acid digestion (AD) and dry ashing) and extraction procedures (such as liquid-liquid extraction (LLE), Soxhlet extraction and solid-phase extraction, SPE). However, these protocols can entail several drawbacks, such as the amount of sample required, the volume of organic and/or toxic reagents and solvents needed, or the extensive use of energy. Thus, these conventional sample preparation approaches evolved toward miniaturized and alternative approaches in order to solve these inconveniences.

In this chapter, miniaturized and alternative approaches will be discussed, including, but not limited to, matrix solid-phase dispersion (MSPD), solid-phase microextraction (SPME), micro-solid-phase extraction ( $\mu$-SPE), liquid-phase microextraction (LPME), small-volume microwave-assisted extraction (MAE), vapor-phase acid digestion (VPAD), vapor-phase microwave-assisted digestion (VPMAD), miniaturized ultrasonic SS, and miniaturized ultrasound-assisted extraction (UAE) and digestion (UAD). 


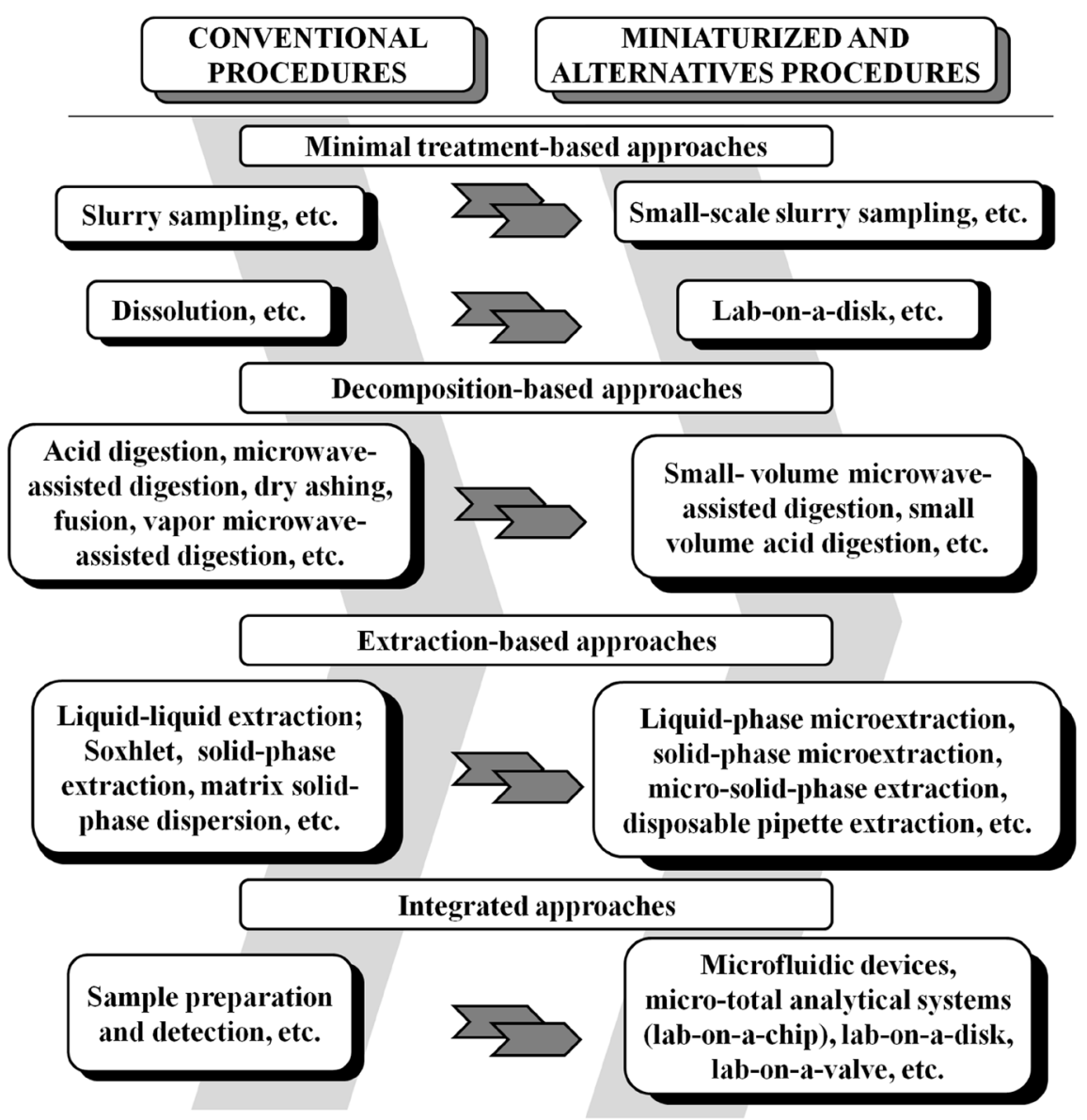

Figure 7.2 Evolution of sample preparation procedures from conventional to miniaturized approaches.

\subsection{Objectives and Benefits of Miniaturized Sample Preparation Procedures}

Recently, miniaturization of classical analytical techniques and procedures has emerged as a new branch of scientific research. Likewise, the trend toward small, autonomous approaches that consume smaller amounts of reagents, require less sample and take less time to process is clear (Pawliszyn, 2003; Felton, 2003). The general objective of miniaturized approaches lies in improving the sample preparation procedures by developing integrated and automated systems, simplified protocols using less amount of sample in a short time and with less energy and reagent consumption. The main principles of miniaturization approaches are summarized in Figure 7.4 . 


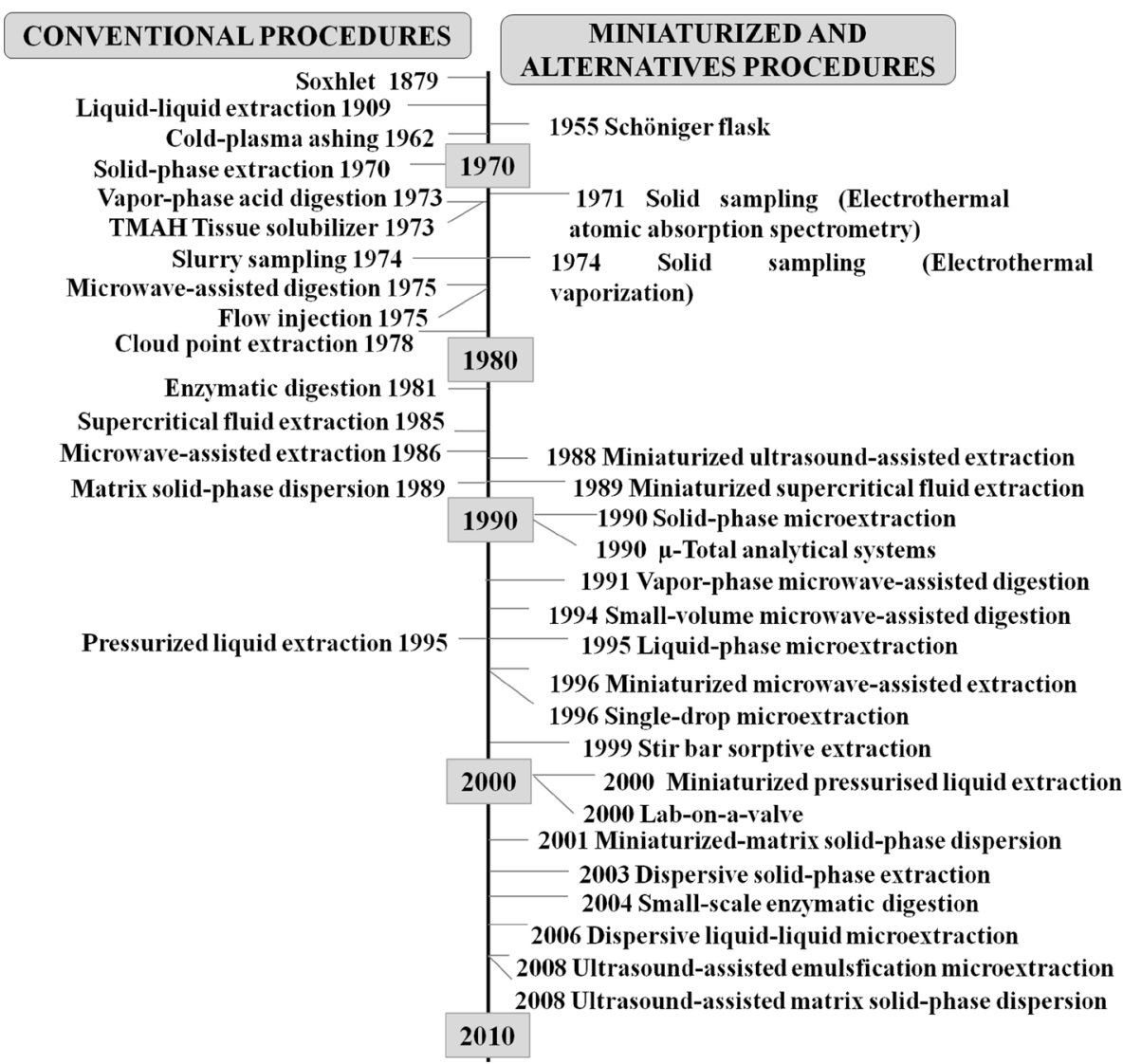

Figure 7.3 Chronological order of appearance of different miniaturized sample preparation procedures compared to conventional methods.

\subsection{Challenges of Solid Sample Analysis}

Sample preparation continues to be a critical step in the analytical process and the most important source of error. In fact, due to the number of steps that are often involved, sample preparation usually takes approximately two thirds of the total analysis time (50-70\%) (Hyötyläinen, 2009) and tends to be slow and labor-intensive. The appropriate choice of the sample preparation procedure should be done according to the main matrix and the analyte of interest. For example, environmental and biota samples are complex matrices, and generally contain a wide variety of compounds in addition to the target analytes (Clement \& Hao, 2010). Thus, sample preparation steps are required to bring the sample into solution, to extract the analyte from the matrix, to avoid matrix interferences and/or to preconcentrate the analyte prior to the analysis. 


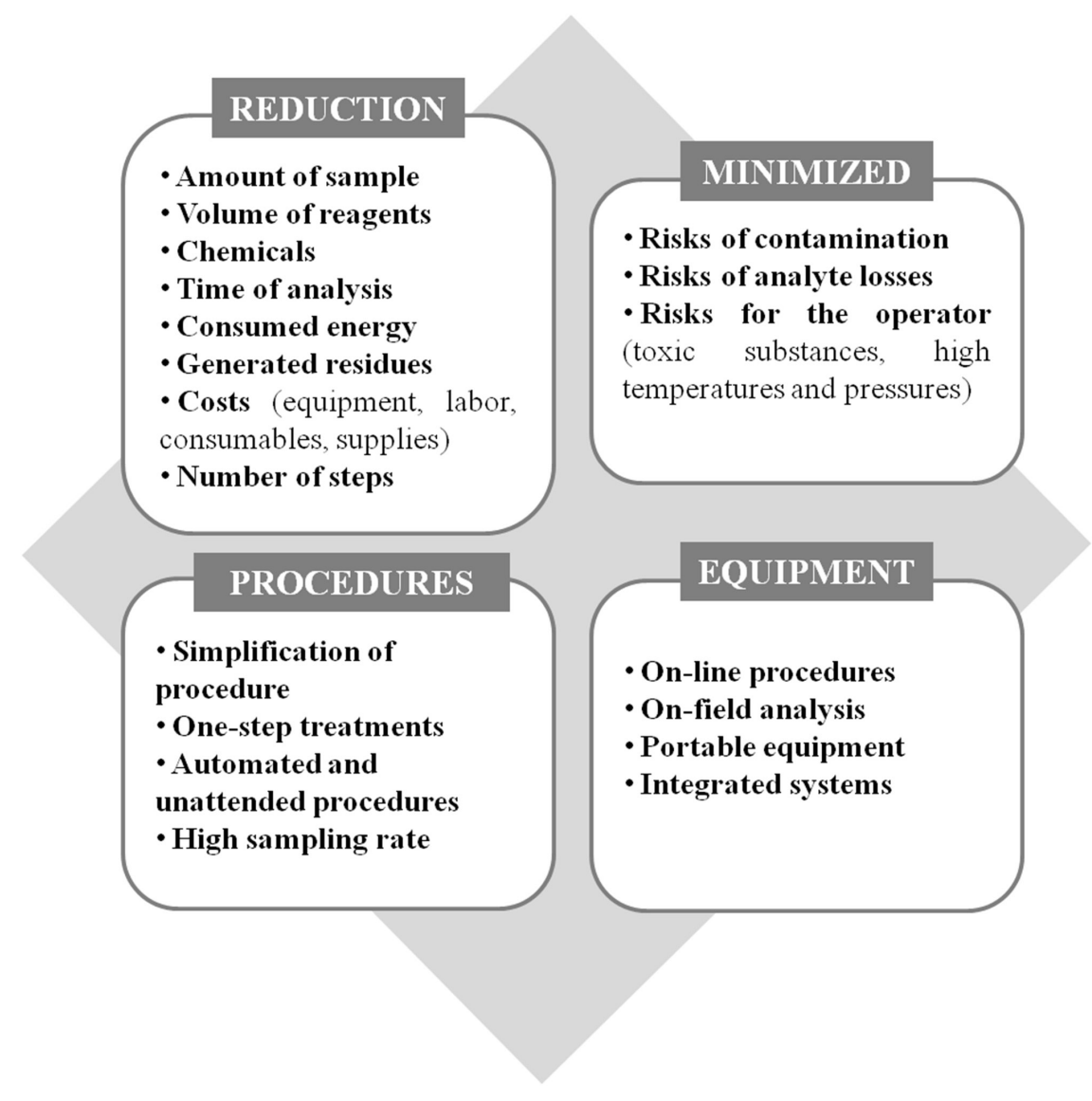

Figure 7.4 Main principles of miniaturized sample preparation procedures.

Solid sample analysis is itself a challenge to both routine analysis and analytical method development due to the complexity of matrices and the very low concentration of target analytes. The main challenges found during sample preparation could be: i) the difficulty of having homogeneous and representative samples, ii) losses of elements by volatilization, iii) contamination of the sample during the processing or storage of the sample, iv) time-consuming and labor-intensive clean-up protocols, v) extensive operator manipulation, vi) difficculty of automation, vii) incomplete dissolution of inorganic samples, and viii) the presence of interferences.

For instance, several errors can occur in the determination of elements in solid samples due to the inherent difficulty in bringing the sample into a solution, including contamination risks, analyte looses and incomplete dissolution (Hoening, 2001). Since solid samples are generally heterogeneous, preliminary treatments are required to obtain a more representative sample with less particle size (Hoenig, 2001). Besides, 
several metals could be retained into the matrix of samples with high silicon content, such as plants, soils and sediments, due to insolubilization phenomena (Hoenig et al., 1998; Hoenig, 2001), being necessary more stringent conditions for sample digestion. Another difficulty consists of deciding if dilution is required, because the approximate concentrations of the analyte and the main matrix components in the sample is usually unknown (Hoenig, 2001).

The determination of organic compounds in biological tissues requires carrying out the disruption of cells and, as a consequence, high concentrations of lipids and proteins are often co-extracted. Therefore, analysis of biological matrices is a tedious process which often gives rise to low recovery and poor reproducibility (Petrovic \& Barceló, 2004). This is the case for Soxhlet-based methods, in which further manipulation can be required. For instance, dirty extracts produced may require the implementation of an extra clean-up step. Besides, the need of large volumes of organic solvents for extraction may require solvent evaporation in order to concentrate the analytes before analysis. In addition, a considerable quantity of co-extracted lipids (levels of lipids can vary widely depending on the tissue) can damage the chromatographic column, particularly in gas chromatography (GC). In fact, several pretreatment steps, such as $\mathrm{AD}$ using $\mathrm{HCl}$, are needed to remove lipids prior to analysis (Clement \& Hao, 2010).

\subsubsection{Types and Composition of Solid Samples}

The target analytes to quantify in solid samples can be very different, including volatile and semi-volatile organic compounds, metals, organometallics and inorganic ions. Moreover, the purpose of the analysis varies from one case to another. For example, analyses can be performed to determine levels of pollutants in an area (e.g., a road, an industry) or a specific sample (e.g., wastes), to evaluate the possibility of reusing wastes for agricultural applications (Osberghaus et al., 1997), to search for bioindicators of pollution, to assess the nutritional composition of food, to evaluate impurities of industrial samples, or to analyze biomolecules related with a disease.

In general, solid matrices are complex and very different in nature and composition. Likewise, each type of sample entails the use of a specific sample preparation procedure. Solid matrices can usually be classified as organic or inorganic regarding their main composition, but in most cases they are a mixture of both types of components. There are samples with high proportion of organic matter (biological tissues, food, plastics) while others are predominantly inorganic in composition (soils, sediments, metals, alloys, fly ash, rocks, sludge, etc.). Other samples that are commonly analyzed include food, clinical, pharmaceutical, industrial and waste samples (Hoenig de Kersabiec, 1996; Hoenig, 2003).

Biological tissues may include human, animal, fish and plant tissues. Biological samples also encompass food, feedstocks, clinical and agricultural samples because 
they consist basically of animal and plant-based materials. A major component of biological tissues is water (e.g., $70 \%$ in mammals). However, water is often removed by lyophilization or drying of the sample in the first steps of sample preparation. According to the different organization levels, biological tissues (from the smallest to the highest level) in increasing size order are composed of atoms (e.g., C, $\mathrm{H}$, $\mathrm{O}, \mathrm{N}, \mathrm{P}, \mathrm{S}$, inorganic ions) < molecules and macromolecules (e.g., proteins, lipids, carbohydrates and nucleic acids) < organelles (e.g., mitochondria, liposome, Golgi apparatus) < cells < tissues (e.g.,neural tissue, epithelial tissue, muscle tissue, connective and supportive tissue) < organs and organ systems (e.g., lung and respiratory system, etc.) < organisms (e.g., humans, fish, animals, plants) (Müller-Esterl, 2008). The main differences between animal and plant tissues are the presence of the cell wall, chloroplasts and silica in plant tissues. The cell wall is mainly composed of cellulose, which is the most abundant organic compound in plants. Moreover, silicon in plants provides structural support and improves tolerance to diseases, drought and metal toxicity (Hodson et al., 2005). Siliceous residues can be found in some plants and plant-based foods. Clinical samples include other tissues such as teeth and bones that present high inorganic content apart from the organic components. The organic matrix of these samples consists of collagen and a small fraction of noncollagen proteins, lipids, citrates and sugars. Biological hard tissues (enamel, dentin and bone) consist of a mineral matrix (hydroxyapatite crystals $\left(\mathrm{Ca}_{5}\left(\mathrm{PO}_{4}\right)_{3}(\mathrm{OH})\right.$ ), water and an organic matrix (Bachmann et al., 2006). Clinical or biomedical samples may include both solid (bone, teeth, hair, nails, tissue) and semi-solid samples (blood, faeces, viscera) (Sansoni \& Panday, 1994) and may also contain organic biomolecules.

Geological samples, soils and sediments are also widely analyzed. These type of samples present a variable organic matter content (from less than $1 \%$ to $40 \%$ of $\mathrm{C}$ ). Soils and sediments are composed of different phases such as organic matter (humic and fulvic acids, organism residues) and minerals (oxides of iron, aluminum and manganese, phyllosilicate minerals, carbonates and sulfides) (Filgueiras et al., 2002a). Silicate rocks comprise a wide range of different mineral species. Moreover, soils without pre-treatment contain a biotic portion that consists of a diverse array of bacteria, fungi, worms and insects (Del Castilho \& Breder, 1997).

On the contrary, solid waste is a comprehensive term and generally implies heterogeneous material, including e.g., industrial waste, domestic refuse, excavated material, wrecked cars, used tires, sewage sludge and numerous other materials discarded by human society (Osberghaus \& Helmers, 1997). It should be pointed out that sewage sludge constitutes a relatively homogenous matrix due to the long deposition time of sludges in sewage treatment plants (Schladot \& Backhaus, 1997). Other waste samples include fly ash and other combustion products, such as industrial powders, solid sewage and industrial wastes (Clement \& Hao, 2010). These industrial solid materials, as well as cements and ceramics, contain refractory compounds such as $\mathrm{Al}_{2} \mathrm{O}_{3}, \mathrm{SiO}_{2}, \mathrm{MgO}, \mathrm{CaO}, \mathrm{Cr}_{2} \mathrm{O}_{3}$ and $\mathrm{ZrO}_{2}$ and can be also complicated matrices (Rechenberg, 1997). 
Apart from the target samples, certified reference materials (CRMs) of different origins are often purchased and analyzed along with unknown samples. These reference materials are used to test and validate newly-developed procedures as they help to establish the accuracy and precision of a methodology. Moreover, CRMs can be used to calibrate instruments and assure analytical quality control. CRMs of most of the previously mentioned types of samples are available in different sources, such as National Research Council of Canada (NRCC, Canada), Community Bureau of Reference (BCR, Brussels, Belgium), National Research Center for Certified Reference Materials (China) and National Institute of Standards and Technology (NIST, USA).

\subsubsection{Pre-treatment of Solid Samples}

The common steps involved in almost every analytical process include sampling, sample transport and storage, sample preservation, sample pre-treatment and sample preparation (or sample treatment) and analysis. These processes are highly linked to the nature of the sample and the analyte and are aimed at minimizing sample heterogeneity and ensuring sample representativeness. Herein, several main types of solid sample pre-treatment will be discussed.

Sample pre-treatment can include processes such as drying, homogenization, extraction, concentration and/or clean-up depending on the sample, the analyte and the analytical technique to be employed (Mitra \& Brukh, 2003). Preservation of samples should avoid physical (e.g., volatilization, diffusion, adsorption) and chemical changes (e.g., photochemical reactions, oxidation, precipitation), as well as biological degradation, with the goal of preventing or minimizing any changes in sample composition (Mitra \& Brukh, 2003). A table of suitable conditions of storage for different analytes can be found in the literature (Mitra \& Brukh, 2003).

In some cases, the analysis of solids may require the initial removal of extraneous matter. For instance, removal of shells of some seafood can be required in the case of analysis of marine organisms when only soft tissue is to be analyzed. In the case of analysis of internal organs, marine or terrestrial animals must be eviscerated. With regard to plants, depending on the anatomical part to be analyzed (i.e., flower, stem, leaf) structures that are not being analyzed must be removed.

Once the specific matrix of interest is selected, another stage of the pre-treatment process includes, in many cases, drying the sample to prevent it from having a high concentration of moisture, which could disturb the analysis or encourage biodegradation (Petrovic \& Barceló, 2004), as is often the case for soil and sediment analysis. The type of drying conditions and the temperature are parameters to take into account at this stage. For example, the ammonium and nitrate content of soil samples can vary significantly depending on whether the sample has been open air-dried or dried in an oven. 
After drying, the sample may need to be reduced in size and mass. Reduction of particle size can be achieved by several processes including, but not limited to, chopping, grinding, mincing, pulverization, blending or mixing. Similar to other cases, care has to be taken to avoid loss of homogeneity or even analyte losses during these processes. For instance, volatile compounds are likely to be released from the solid matrix throughout these stages. This step is especially critical when it comes to the analysis of low mass samples, since sample representativeness could be lost during the process. In this regard, the minimum sample mass intake that ensures homogeneity should be determined.

Storage and sample preservation is also an important issue, especially for those solid samples that will not be immediately analyzed after preparation. Temperature (and therefore stability), oxygen/inert atmosphere, light and storage material conditions have to be established to ensure sample integrity. In all cases, sample headspace should be minimized to avoid degradation. In general, a fast transportation to the analytical laboratory is of high importance, especially for the analysis of fresh products such as edible matrices or biological tissues, whose molecular degradation could lead to a loss of sample integrity (Pawliszyn, 2002).

\subsubsection{Extraction Mechanisms}

The mechanisms that govern the extraction of analytes from the matrix are much more complicated when the matrix is a solid. Since the analyte has to be extracted from the matrix and then transported into different phases, numerous processes take part in the extraction mechanism. In this regard, a comprehensive chapter on the theory of extraction has been published by Pawliszyn as a part of a book aimed at sample preparation. All these mass transfer processes can be expressed through mass balance equations, which are modified for those energy-, pressure- and temperatureassisted techniques for the preparation of solid samples (Veggi et al., 2013).

Basically, diffusion of the extractant through the matrix, solvation and interactions with the matrix components, diffusion of the analyte out of the solid and migration from the surface matrix towards the bulk extractant are the main processes occurring during the extraction of the analyte from the solid sample matrix. In this process, partitioning of the analyte depends on its affinity towards the surrounding media and, therefore, the extractant must be able to establish strong interactions with the analyte, enough to overcome those forces keeping the analyte bound to the matrix. In this sense, both physical adsorption to the matrix and solid matrix-analyte chemical interactions (e.g., van der Waals forces, electrostatic interactions and hydrogen bondings) play an important role.

In addition, extractions using sorbent-based techniques must consider the specific sorption and desorption mechanisms between the analyte and the sorbent. The characteristics of the solid sample, the physico-chemical interactions of the analyte 
(inorganic or organic) with the matrix, along with the characteristics of the phase used for extraction also influence the mechanism. In most cases, the extractant phase to sample ratio should be high to ensure complete extraction, especially in the case of exhaustive extraction techniques (e.g., Soxhlet extraction).

\subsection{Sample Preparation Techniques for Solid Samples: from Con- ventional to Miniaturized Alternatives}

Preparation of samples for metal and organic compound determination at trace levels is applied to i) degrade or solubilize the matrix, ii) dilute the matrix and iii) extract, separate or concentrate the analyte (Mitra \& Brukh, 2003). Sometimes, it is necessary to destroy the organic matter (including proteins, lipids and carbohydrates) in order to determine inorganic compounds. Usually, sample preparation for trace analysis requires more sophisticated procedures than major component analysis. Whereas carrier gas hot extraction or combustion can be enough for the analysis of major components, additional clean-up and preconcentration techniques (i.e., SPE of LLEbased techniques) are required in many cases for trace analysis, some of them even using specific instrumentation as in the case of pressurized liquid extraction (PLE) or supercritical fluid extraction based approaches (Mitra \& Brukh, 2003).

As discussed above, one of the trends in sample preparation is the downsizing of analytical methodologies. Miniaturization of analytical systems is performed at small and very small scales. A possible classification of the different devices into three levels could be acheived based on the size of the devices and the volume of sample used: mini- (from $\mathrm{cm}$ to $1 \mathrm{~mm}$ and $\mu \mathrm{L}$ ), micro- (lower than $1 \mathrm{~mm}$ and $\mathrm{nL}$ ) and nano(lower than $1 \mu \mathrm{m}$ and pL, fL, aL) devices or systems (Ríos et al., 2009).

Some problems inherent to miniaturization include sampling, sample preparation and sample introduction, especially in the case of solid samples (Rios et al., 2009). More research is needed in these fields of study.

In this section, different conventional and miniaturized sample preparation procedures for solid and semi-solid samples are discussed. Different classifications have been compiled for organic and inorganic analytes. In Table 7.1, an assessment of different strategies for miniaturized sample preparation according to their 'miniaturization profile' for metals, organometallics and organics analytes is provided.

\subsubsection{Trace Elemental and Organometallic Analysis}

Several procedures mainly employed for trace elemental and organometallic analysis are described in this section. The evolution from conventional (more established procedures) to miniaturized procedures is also discussed. Table 7.2 shows selected applications of miniaturized sample preparation for metals and organometallics. 


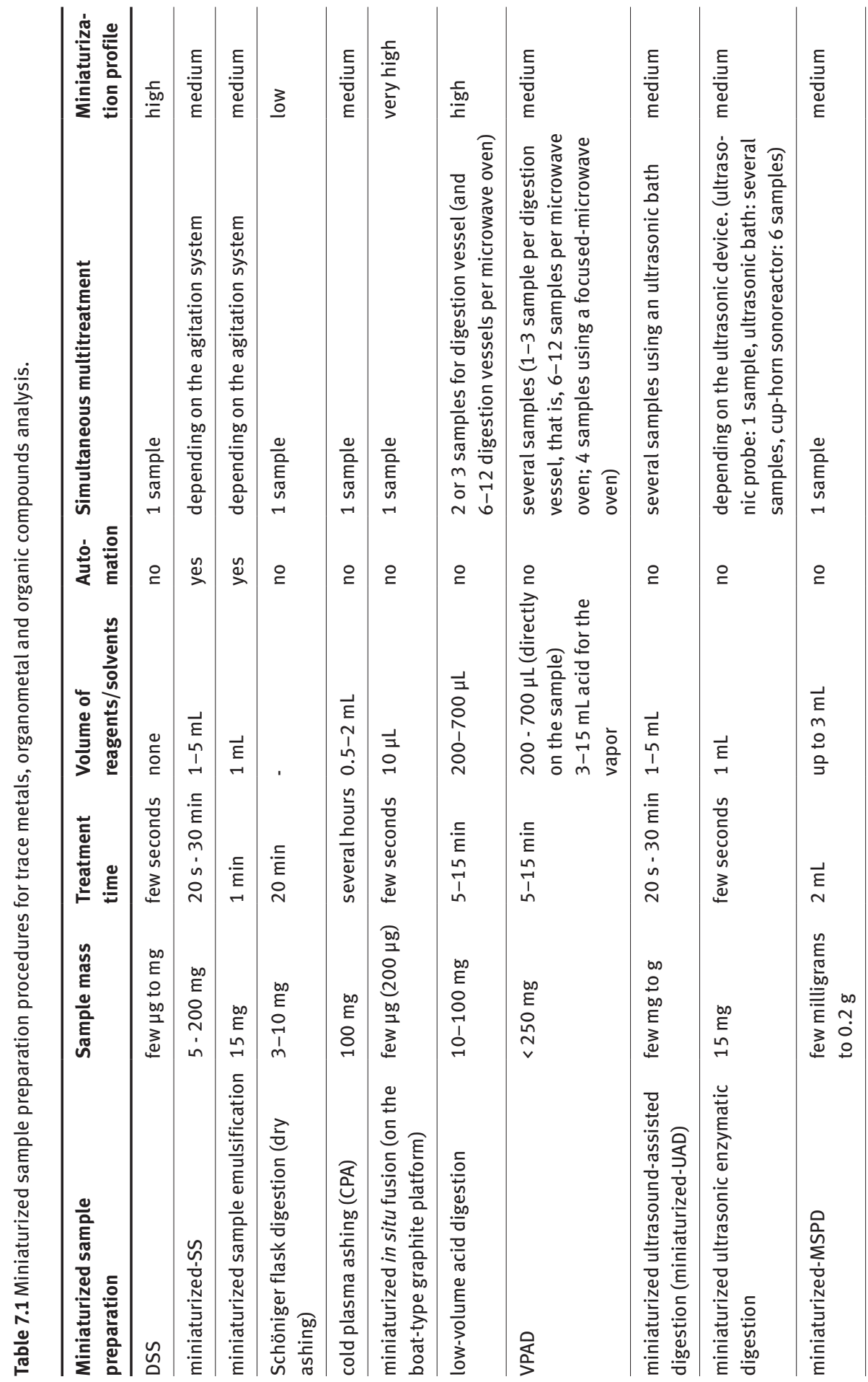




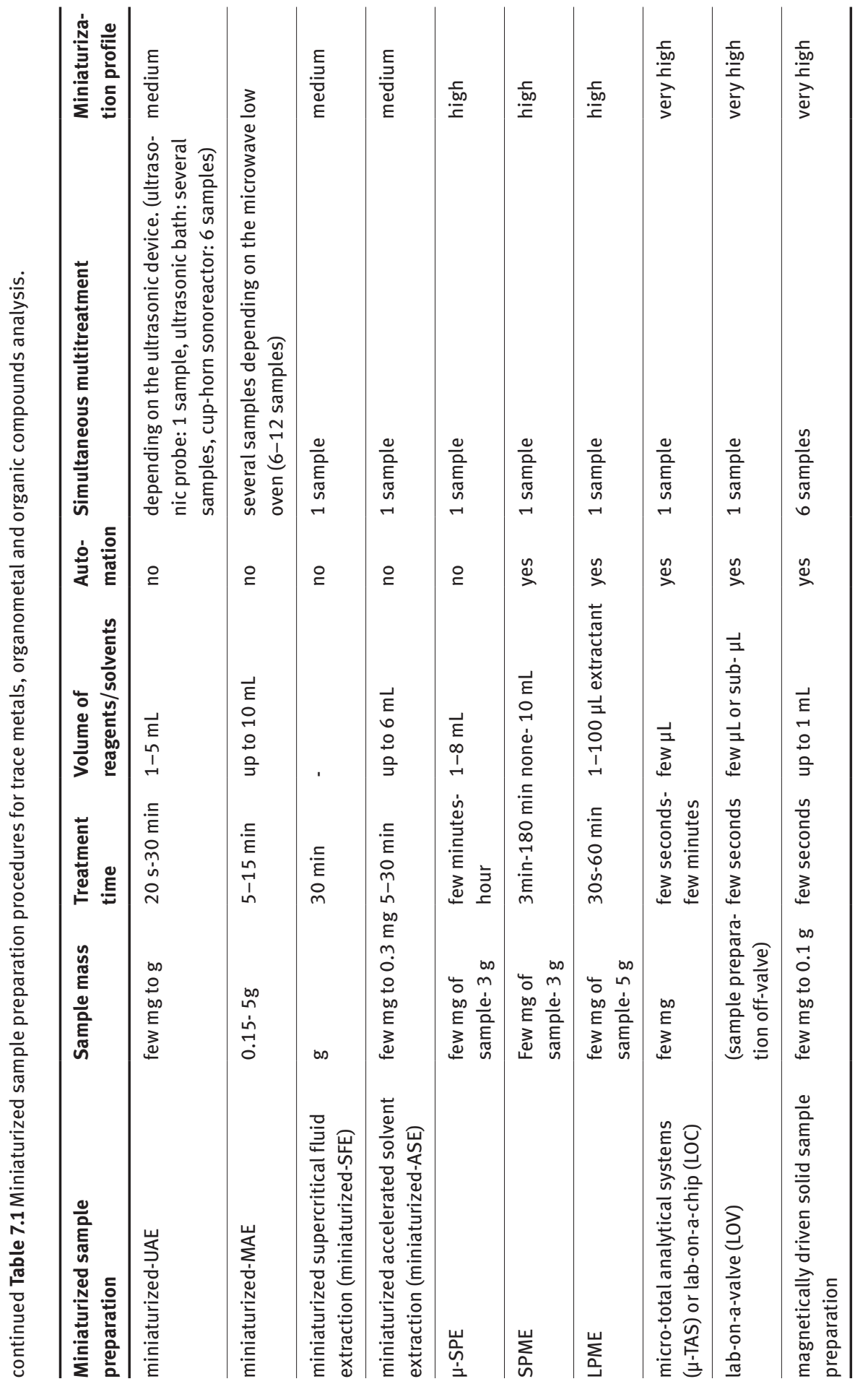




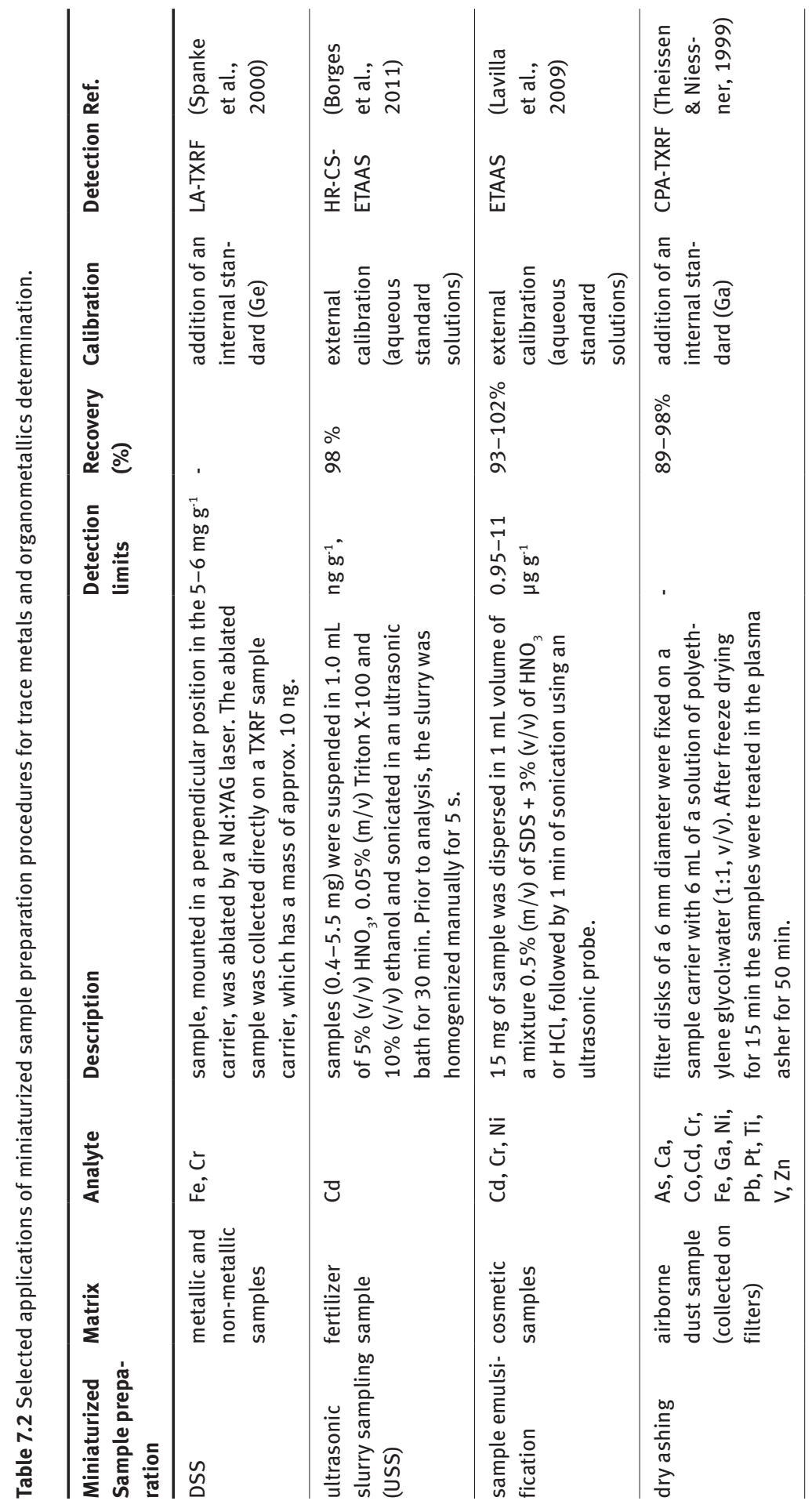




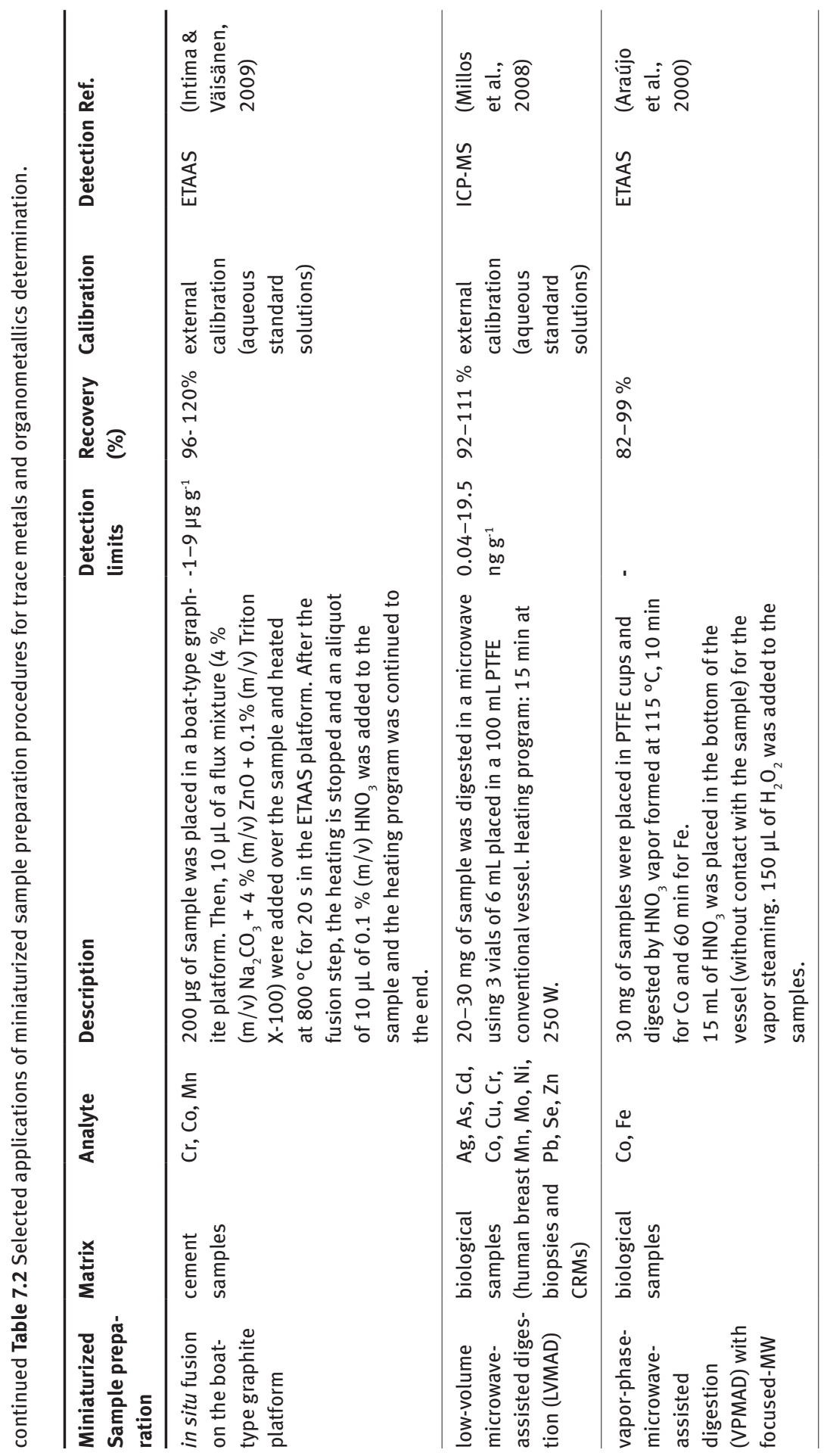




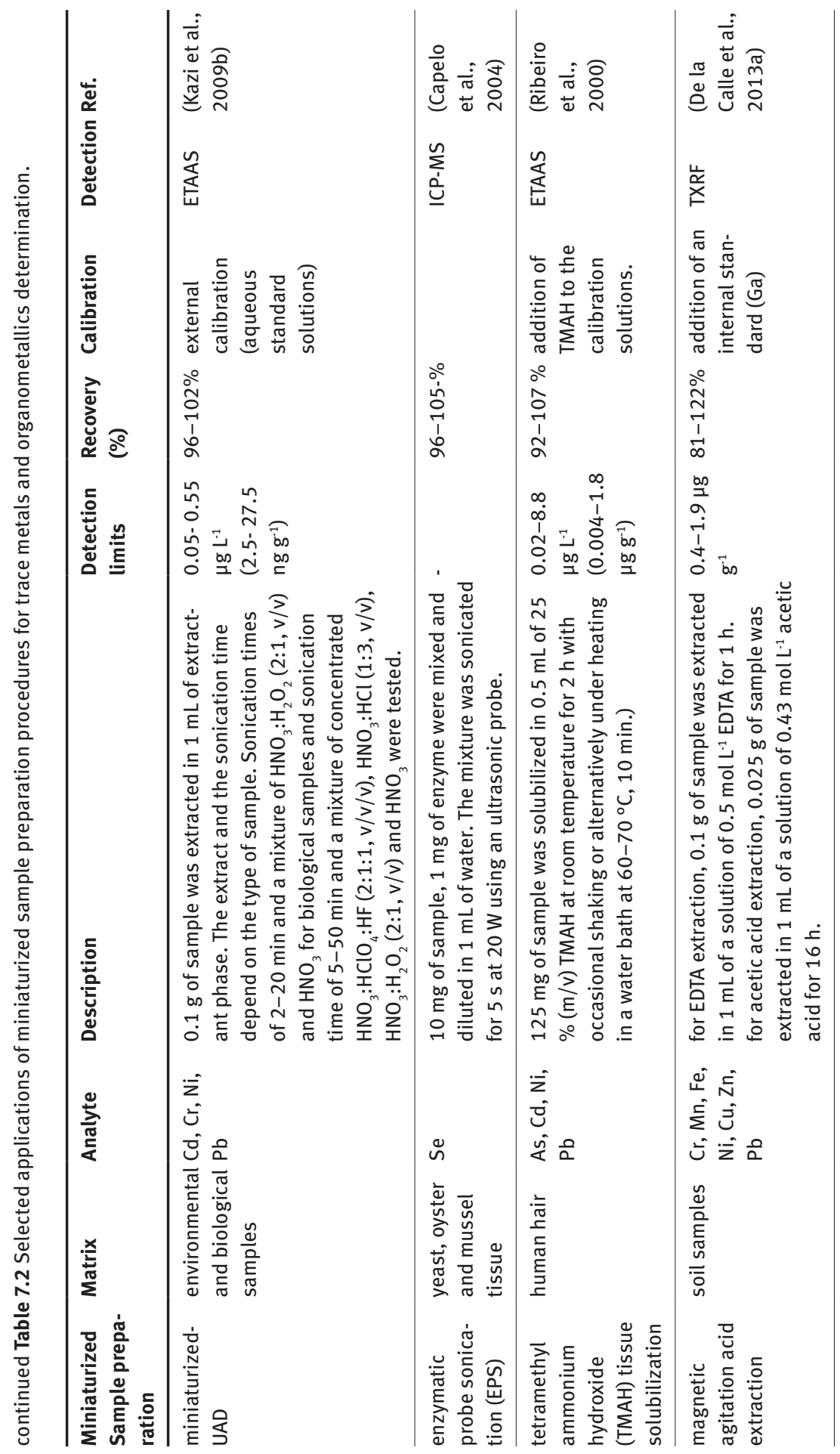




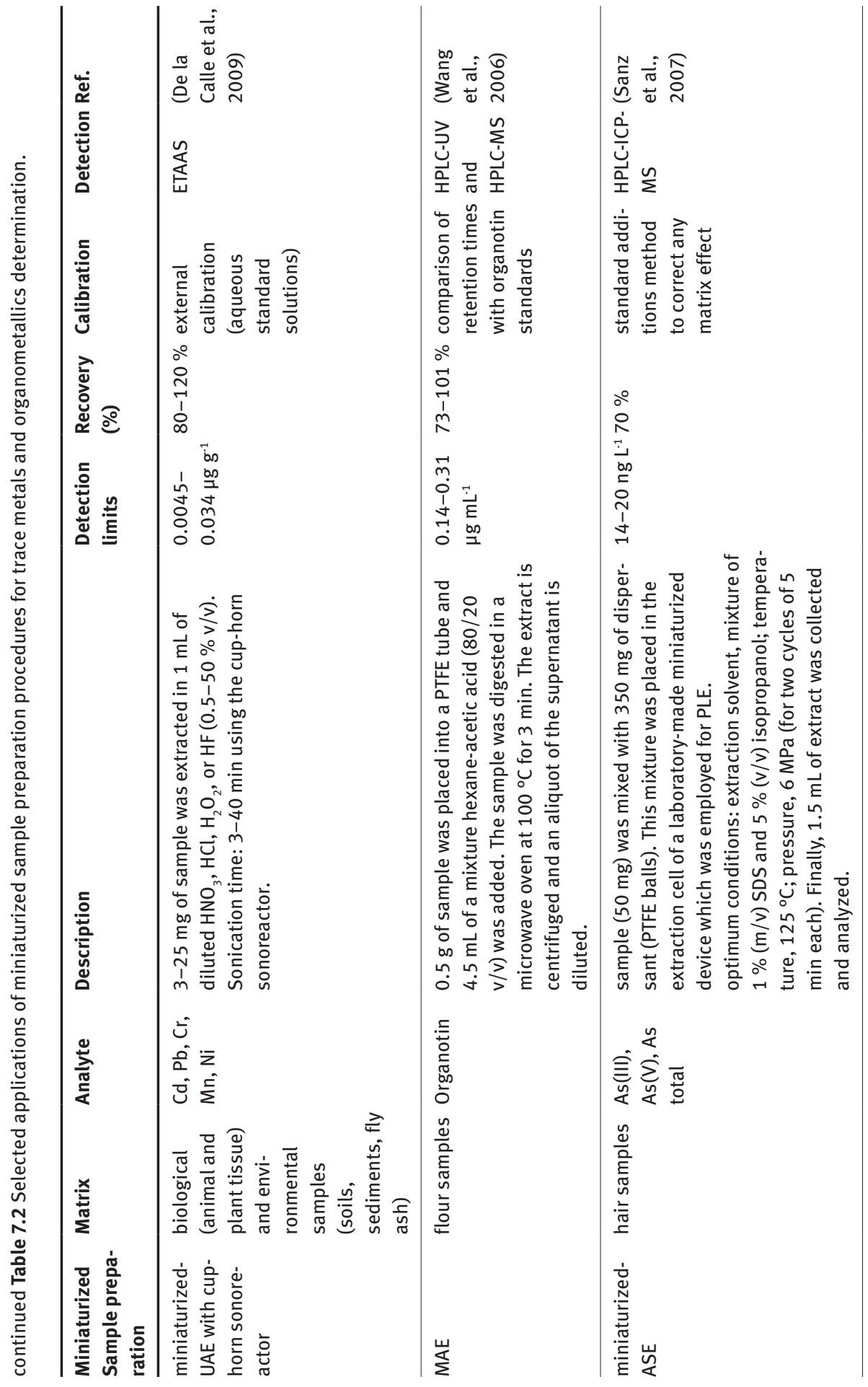




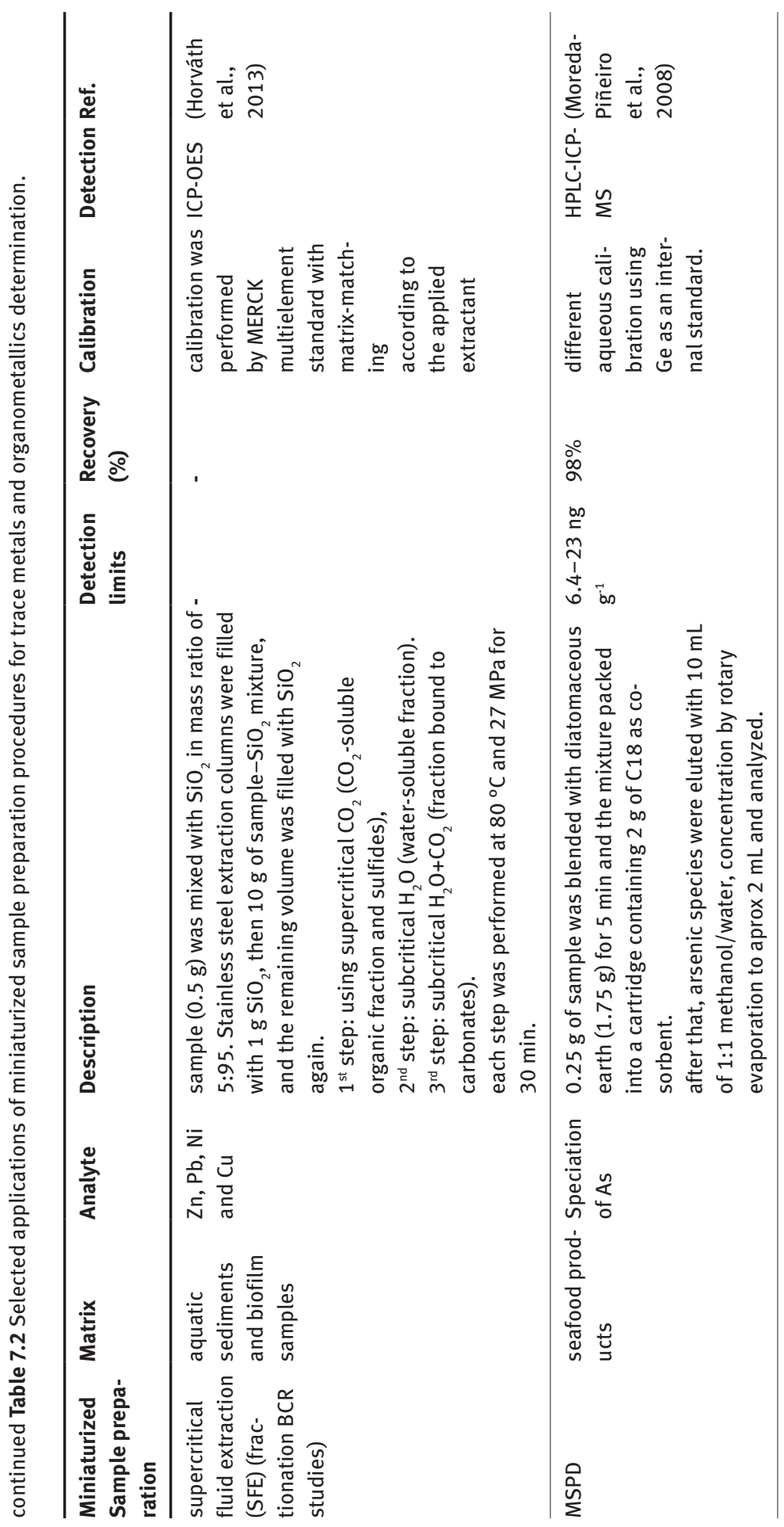




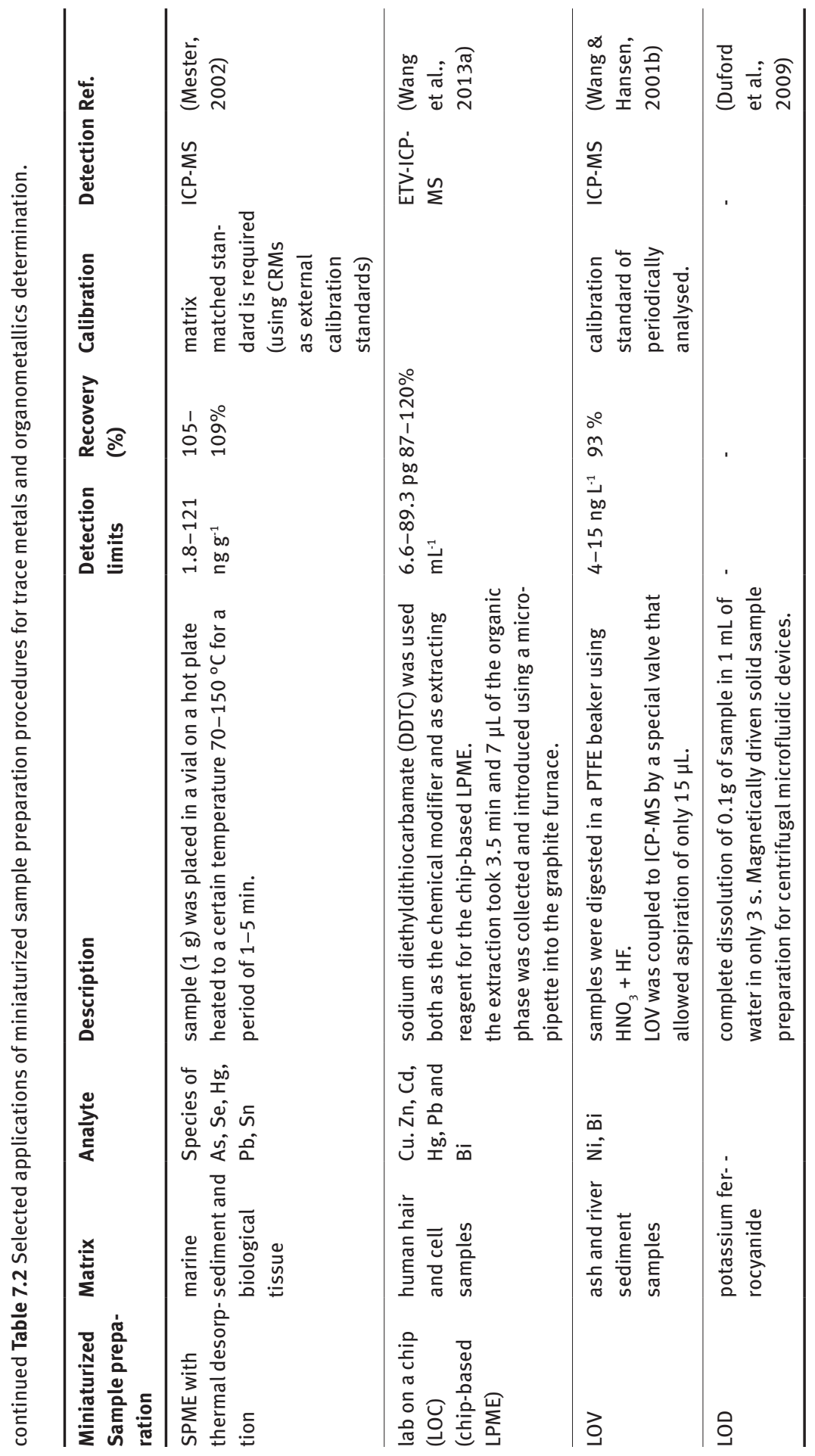




\subsubsection{Minimal Treatment-based Techniques}

As discussed above, sample treatment is one of the most energy- and reagent- consuming steps of the analytical process, and the one with the greatest risk of sample contamination and analyte losses. Non-intensive procedures avoid: i) time-consuming steps of decomposition or extraction of the solid sample, ii) the use of large volumes of acids and organic solvents, iii) generation of additional wastes and iv) risks or hazards to the operator. Here, procedures without treatment (direct solid sampling, DSS) and with minimal sample treatment (SS and emulsification) are presented.

\subsection{Direct Solid Sampling}

As discussed in the introduction section, there are several analytical techniques that can directly analyze a solid sample. Classically, electrothermal atomic absorption spectrometry (ETAAS) using specially-designed graphite tubes (Bendicho \& de Loos-Vollebregt, 1991) and laser-induced breakdown spectroscopy (Yamamoto et al., 1996; Capitelli et al., 2002) were applied for the direct analysis of solid samples. More recently, glow discharge optical emission spectrometry (Bengtson \& Lundholm, 1988; Pisonero et al., 2006), high resolution continuum source ETAAS (HR-CS-ETAAS) (Welz et al., 2007), laser ablation (LA)-ICP-MS (Günther, 2005; Arroyo et al., 2009) and electrothermal vaporization with inductively coupled plasma mass spectrometry (ETV-ICP-MS) (Vanhaecke et al., 2002), and inductively-coupled plasma optical emission spectrometry (ETV-ICP-OES) (Matschat et al., 2005) have gained more attention. Other possibilities for direct solid analysis are instrumental neutron activation analysis (Kolmogorov et al., 2009), X-ray fluorescence spectrometry (XRF) (Khuder et al., 2009) or total reflection X-ray fluorescence spectrometry (TXRF) (Magalhaes et al., 2010; Vázquez et al., 2010).

Conversely, direct solid sample analysis is not usually combined with flame atomic absorption spectrometry (FAAS), ICP-OES and ICP-MS, since it is necessary to prepare (pre-treat and treat) the sample prior to the analysis. In the case of FAAS, this occurs due to the insufficient dissociation of solid particles in the relatively cold flame; while in the case of ICP-OES and ICP-MS, it is due to physical and chemical interferences (Hoenig \& de Kersabiec, 1996).

Some of the advantages of DSS analysis are i) higher sensitivity (no dilution), ii) faster analysis without sample preparation, iii) reduced risks of contamination and analyte loss, iv) hazardous reagents are not required, v) reduced generation of wastes and vi) reduced hazards for the operator. However, some of the limitations are: i) inhomogeneity of solid samples (due to the small sample size), ii) less precise results and iii) the need of CRMs (Bendicho \& de Loos-Vollebregt, 1991; Welz, 2007).

From the point of view of miniaturization, ETAAS, ETV-ICP-OES or ETV-ICP-MS, LA-ICP-MS and TXRF are microanalysis techniques since only a very small amount of solid sample is employed. ETV consists of a simple combination of a graphite tube as an ETV device with ICP-MS or ICP-OES. The aim of using ETV consists of achieving selective atomization/vaporization of the analyte (Belarra et al., 2002). LA generates 
a vapor-phase aerosol using a pulsed laser beam focused on a sample surface (Russo \& Baldwin, 2003; Potts \& Robinson, 2003; De la Guardia \& Armenta, 2011). Thus, LA offers a simple form to deal with materials that are difficult to digest or dissolve such as alloys and refractory materials. Another miniaturized approach was applied using LA directly onto quartz sample carriers of TXRF (Bredendiek-Kamper et al., 1996; Spanke et al., 2000) (Figure 7.5A). The main advantages involved in this last procedure are no sample preparation, minimum risks of contamination and the possibility of local and micro-distribution analysis.

While techniques such as XRF require high amount of sample (10 g), lower amounts of samples are enough in some other techniques: $0.5-3 \mathrm{mg}$ (typically $1 \mathrm{mg}$ ) in ETV-ICP-MS (Vanhaecke et al., 2002) and ETV-ICP-OES (Matschat et al., 2005), $0.5 \mathrm{mg}$ of medicinal plants by HR-CS-ETAAS (Figueredo-Rego et al., 2012), a few $\mu$ g of tissue or powder sample by TXRF (Magalhaes et al., 2010; Vázquez et al., 2010), 0.1-10 $\mu$ g in LA-ICP-MS or LA-ICP-OES (De la Guardia \& Armenta, 2011) and a few ng of steel and ceramic samples in LA-TXRF (Bredendiek-Kamper et al., 1996; Spanke et al., 2000).

\subsection{Slurry Sampling}

SS is an alternative for direct solid analysis and provides a faster and cheaper option for sample preparation than decomposition approaches. Several problems derived from the use of DSS, such as the inhomogeneity, can be alleviated using SS (Hsu et al., 2013). The main advantages of SS include: i) ease of sample preparation, ii) use of non-corrosive reagents, iii) reduced risks of loss of volatile elements and iv) reduced risks of contamination. Additionally, it does not require complete dissolution of the sample, which is a great advantage in case of samples that are difficult to dissolve.

SS involves the preparation of a suspension of powdered sample in a liquid medium and several reviews on this topic have been published (Bendicho \& de LoosVollebregt, 1991; Cal-Prieto et al., 2002; Ferreira et al., 2010). Preparation of slurries can involve partial decomposition and extraction, particularly if acids are present in the suspension medium (Ihnat, 2003).

Several parameters should be studied when SS is applied, including the i) type of diluents, ii) stabilizing agents, iii) homogenization systems, iv) particle size and v) sample mass-to-volume ratio.

Usually, the liquid medium consists of a dispersant or surfactant (e.g., Triton X-100, Triton X-114) in the presence of a highly diluted acid to avoid flocculation of particles. In other instances, reagents such as diluted glycerol, ethanol, hexametaphosphate, or TMAH were used as stabilizing agents.

Apart from manual agitation (Ferreira-Damin et al., 2011), different systems are commonly used in order to obtain a homogeneous suspension, such as magnetic agitation, vortex, Ultraturrax and gas mixing, or a combination of systems. For instance, the application of Ultra-turrax homogenizer ( $5 \mathrm{~min}$ ) and vortex agitation (1 $\mathrm{min}$ ) was performed for analysis of baby food samples (Ozbek \& Akman, 2012). More recently, 


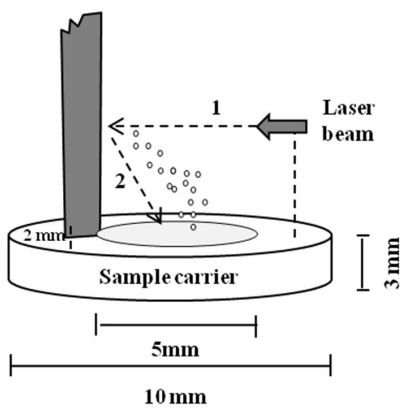

(A)
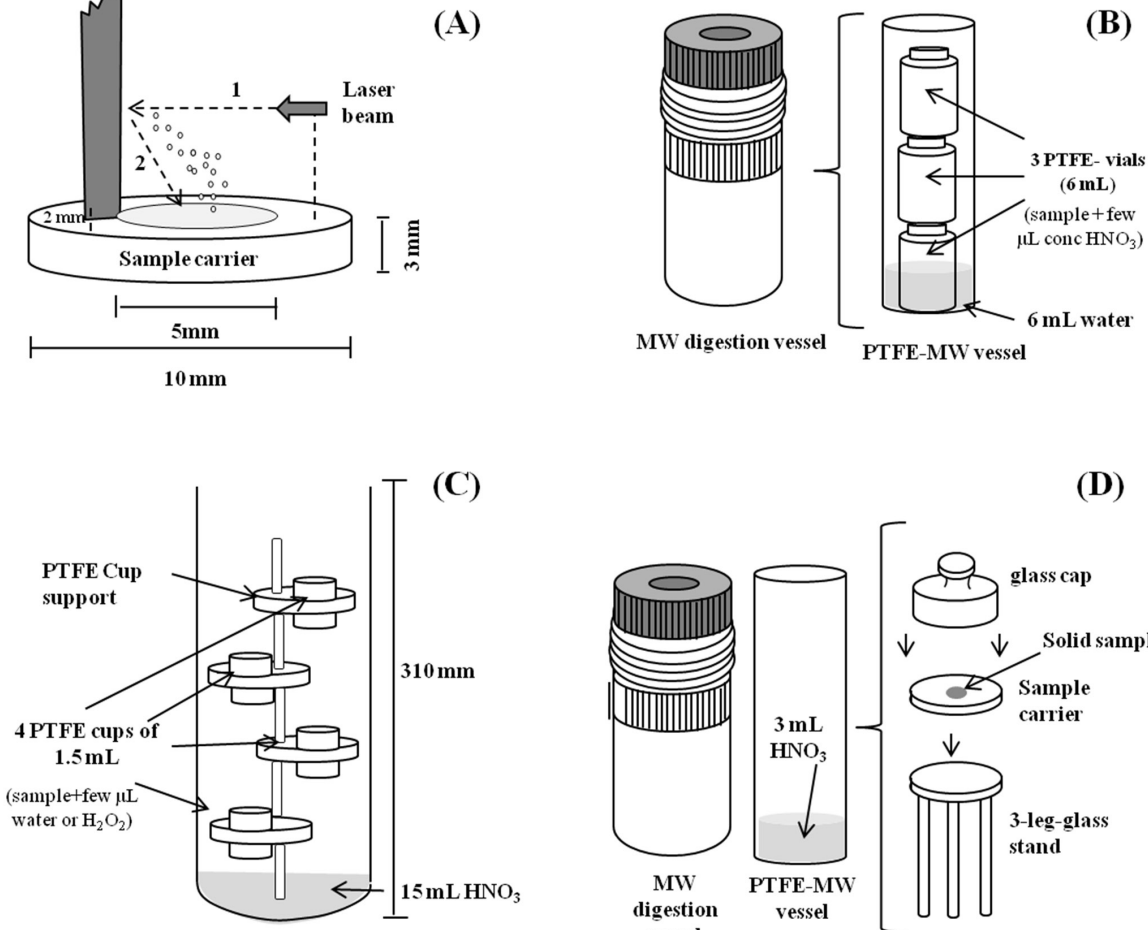

Focused microwave vessel

(glass)
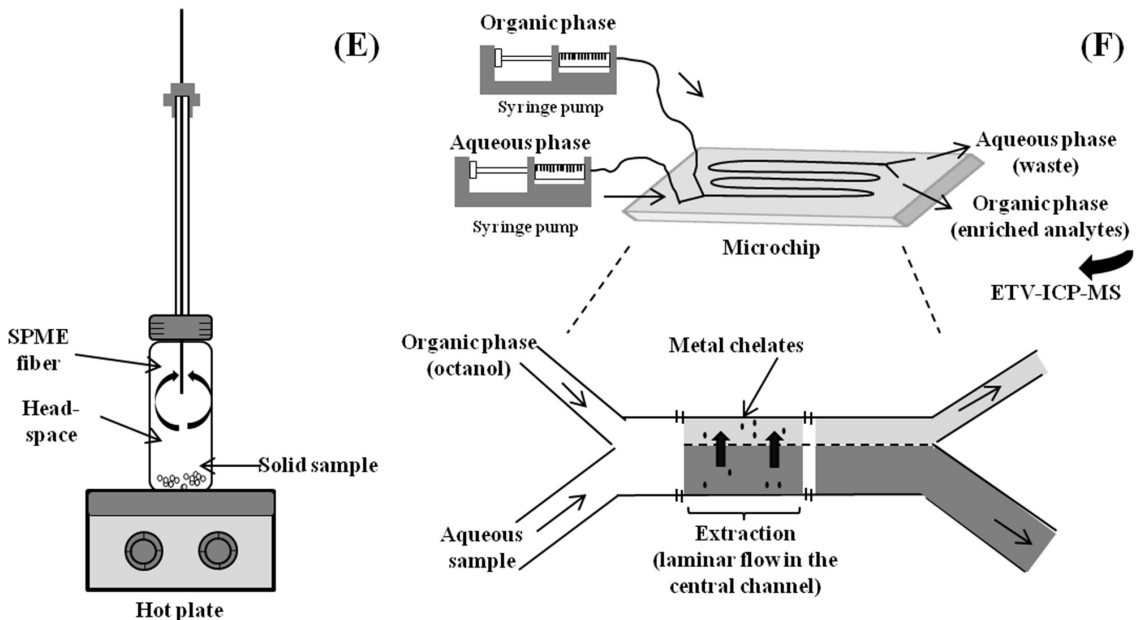

Figure 7.5 Examples of miniaturized sample preparation procedures for determining metals in solid samples. (A) DSS (1. Laser ablation and 2. Deposition onto the sample carrier). (B) Small-vessel microwave-assisted digestion. (C) VPMAD in a focused microwave (FMW) vessel. (D) VPMAD in TXRF sample carriers. (E) Headspace extraction of solid on a SPME fiber. (F) Chip-based LPME. 
ultrasound energy was applied for this purpose since this type of energy can drive slurry mixing processes. Generally, ultrasonic probes are preferred due to the high intensity and low time (few seconds) that is required to form a homogenous slurry. On the contrary, when an ultrasonic bath is applied, higher sonication times (15-30 min) are required due to the lower intensity, (Vignola et al., 2010; Kadenkin et al., 2011; De Jesus et al., 2013).

Particle size is also a key parameter for slurry stability and homogeneity. High particle sizes $(\geq 300 \mu \mathrm{m})$ provide less reproducibility and sensitivity while a particle size of $<50 \mu \mathrm{m}$ seems to be adequate for SS (Cal-Prieto et al., 2002). A grinding step is usually required to obtain a homogeneously-distributed slurry. Generally, the appropriate sample mass-to-volume ratio is less than 0.25 (Miller-Ihli, 1993). Typically, a sample mass in the range of 5-200 $\mathrm{mg}$ and a volume of diluents in the range of 1-100 mL are employed.

In spite of being a classical sample preparation approach (Brady et al., 1974), SS procedures have been reported very recently (De la Calle et al., 2012, 2013a; Hsu et al., 2013). As early as 1974, Brady et al. (1974) developed a very fast approach using small amounts of sample and reagents (e.g., $3 \mathrm{mg}$ of sediment in $10 \mathrm{~mL}$ of water, vortexmixed for $20 \mathrm{~s}$ ).

The main trends in SS are focused on miniaturization and acceleration by application of ultrasound energy. Miniaturized SS has been applied based on reducing the mass of sample and the volume of reagents. Recent publications include the trace elemental analysis of biological tissues (De la Calle et al., 2012) and soils (De la Calle et al., 2013a). The amount of sample employed in the most recent publications range from 0.4 to $50 \mathrm{mg}$, while the volume of suspension ranged from $700 \mu \mathrm{L}$ to $3 \mathrm{~mL}$ (Sánchez-Moreno et al., 2009; Vignola et al., 2010; Borges et al., 2011; Ferreira-Damin et al., 2011; De la Calle et al., 2012, 2013a; Scaccia \& Mecozzi, 2012; Dobrowolski et al., 2013a). The agitation systems generally used were manual agitation (Ferreria-Damin et al., 2011), magnetic agitation (De la Calle et al., 2012, 2013a), vortex (Dobrowolski et al., 2013), ultrasonic bath (Vignola et al., 2010; Borges et al., 2011; Scaccia \& Mecozzi, 2012) and ultrasonic probe (De la Calle et al., 2012, 2013a). The agitation time depends on the agitation system employed, and it ranges from 20-30 s (SánchezMoreno, 2009; De la Calle et al., 2012, 2013a), to 30 min (Vignola et al., 2010; Borges et al., 2011; Scaccia \& Mecozzi, 2012).

Other improvements on SS are related with the combination with instrumentation, automation, use of permanent modifiers and preconcentration. First, SS has been combined with multiple analytical techniques (especially multi-elemental techniques). Some examples of these combinations include USS with ETV-ICP-MS (Hsu et al., 2013; Lin \& Jiang, 2013), cold-vapor atomic absorption spectrometry (CVAAS) (De Jesus et al., 2013), ETAAS (Sánchez-Moreno et al., 2009; Ferreira-Damin et al., 2011; De Paula et al., 2012), USS-HR-CS-ETAAS (Vignola et al., 2010; Borges et al., 2011), ETVICP-OES (Amberger, 2010), USS-FAAS (Ozbek \& Akman, 2012), TXRF (Kadenkin et al., 2011; De la Calle et al., 2012, 2013a). As for automation, sample introduction systems 
have included autosamplers equipped with ultrasonic probe slurry samplers since the 1990s (e.g., the USS-100 Ultrasonic slurry sampler from Perkin Elmer) (Amoedo et al., 1999; Santos et al., 2000; Bendicho et al., 2012b). Some examples can also be found in recent publications (Hsu et al., 2013; Lin \& Jiang, 2013). Another specific improvement of SS analysis for ETAAS and ETV-ICP-MS consists of the use of permanent modifiers in the graphite tube, such as $\mathrm{Nb}$ and Ir (Dobrowolski et al., 2013) for $\mathrm{V}$ determination and $\mathrm{Pd}$ nanoparticles for $\mathrm{Zn}, \mathrm{As}, \mathrm{Cd}, \mathrm{Sb}, \mathrm{Hg}$ and $\mathrm{Pb}$ determination by ETV-ICP-MS (Yi et al., 2012). Finally, another interesting strategy that consists of using

SS for preconcentration by means of solid sorbents (Dobrowolski et al., 2012) will be described in section 7.4.1.4.1.

\subsection{Sample Emulsification}

Sample emulsification was proposed as a simple sample treatment for semi-solid liposoluble samples prior to atomic spectrometry (Burguera \& Burguera, 2012). Target samples included oils (De Jesus et al., 2008) and cosmetics (such as shampoo, gel and cream) (Salvador et al., 2000). Sample emulsification is based on forming an emulsion of the semi-solid sample. Therefore, this process provides the sample with similar properties to an aqueous solution (Carballo et al., 2013), being more suitable to be analyzed directly or after dilution. This facilitates the use of aqueous standard solutions for calibration purposes. Emulsification avoids the use of strong acids and large volumes of organic solvents. Moreover, it is a rapid and simple sample preparation procedure with low manipulation of the sample and low generation of wastes compared to other procedures that are commonly employed for these type of samples (e.g., acid dissolution and/or digestion using heating (Adeyemo et al., 2004), ultrasound irradiation (Bangroo et al., 1995; Sanz-Segundo et al., 1999) and microwave energy (Bellido-Mila et al., 2002)).

Similar to SS, several parameters should be optimized, including: i) the type of dispersion medium (surfactants, diluents), ii) the sample mass-to-dispersion medium and iii) homogenization systems.

Conventional approaches involving magnetic stirring and heating have been reported in the literature for $\mathrm{Na}, \mathrm{K}, \mathrm{Ca}, \mathrm{Mg}, \mathrm{Zn}$ and Fe determination in eggs by FAAS (Ieggli et al., 2010) and for oils, margarine and butter analysis by FAAS (Na, K, Ca, Mg, $\mathrm{Zn}$ and $\mathrm{Fe}$ ) and ETAAS (Cr, Ni, As, Pb, Cd, Cu, Mn) (Ieggli et al., 2010).

Mechanical and manual agitation are generally used for emulsion formation and stabilization (Salvador et al., 2000). However, the use of ultrasound energy allows one to obtain more stable and homogeneous emulsions in a faster way than conventional shaking (Luque de Castro \& Priego-Capote, 2007). The use of ultrasound energy accelerates the process of mass transfer between the two immiscible phases thus generating smaller sized droplets of the dispersed medium. This phenomenon occurs due to processes such as interfacial instability of the oil-water interface and transient cavitation bubbles that generate micro streaming, high-pressure shockwave and high local temperature during their collapse (Cucheval \& Chow, 2008). When ultrasound 
energy is employed, the approach is named ultrasound-assisted emulsification. In addition to the above mentioned parameters, sonication amplitude and sonication time should be studied. Ultrasonic probes are generally used instead of ultrasonic baths due to the higher intensity of the former.

Ultrasound-assisted emulsification was proposed as a simple sample treatment for semi-solid cosmetic samples (e.g., shampoo, gel, cream and oils) prior to atomic spectrometry and trace elemental analysis (As, $\mathrm{Cd}, \mathrm{Cr}, \mathrm{Cu}, \mathrm{Hg}, \mathrm{Mg}, \mathrm{Mn}, \mathrm{Ni}, \mathrm{Sr}$ and Zn) (Lavilla et al., 2009). Lavilla et al. employed two different procedures (large and small scale) depending on the analytical technique used. In the large scale procedure, 0.05-0.2 g of sample was dispersed in a $15 \mathrm{~mL}$ volume for FAAS, CVAAS and ICP-OES analysis. On the contrary, in the small scale procedure, $15 \mathrm{mg}$ of sample was dispersed in a $1 \mathrm{~mL}$ volume for ETAAS analysis. The dispersion medium consisted of a mixture of $0.5 \%(\mathrm{~m} / \mathrm{v})$ of SDS $+3 \%(\mathrm{v} / \mathrm{v})$ of $\mathrm{HNO}_{3}$ or $\mathrm{HCl}$ which was sonicated for 1 min using an ultrasonic probe, which produced a stable emulsion.

Recent approaches applied to the analysis of lubricating oils also used a miniaturized procedure after dissolution of the sample in toluene $(0.25-0.35 \mathrm{~g}$ of sample in $10 \mathrm{~mL}$ of toluene). The emulsions were prepared directly in an autosampler cup (1 mL) for ETAAS analysis, adding an aliquot of the dissolved sample and $\mathrm{HNO}_{3}$, followed by manual agitation ( $1 \mathrm{~min}$ ), ultrasonic bath agitation ( $5 \mathrm{~min}$ ) and subsequent addition of xylene + Triton X-100 with additional manual agitation (1 min) (Carballo et al., 2013). Another experiment involved the preparation of a three-component solution directly in an autosampler cup (1 mL). A small aliquot of dissolved sample was placed into the autosampler cup followed by $200 \mu \mathrm{L}$ of methyl isobutyl ketone (MIBK) and $\mathrm{HNO}_{3}$ each, and $100 \mu \mathrm{L} \mathrm{HCl}$ were added. The mixture was sonicated in an ultrasonic bath (15 $\mathrm{min}$ ) and after adding propan-1-ol, the mixture was sonicated in an ultrasonic probe (10 s) just before the analysis (Carballo et al., 2013).

\subsubsection{Decomposition-based Techniques}

In this section, different procedures to bring a solid sample into solution will be described. Since these procedures are undesirable from an environmentally-friendly point of view, several procedures evolved toward miniaturization with subsequent savings in energy, reagents and time. Evolution from the point of view of miniaturization will be taken into account. These procedures include dry ashing, AD, and digestion accelerated by means of microwave and ultrasound energy, enzymatic digestion and tissue solubilization.

\subsection{Dry Ashing}

Dry ashing and dissolution of the residue with dilute inorganic acids (usually $\mathrm{HCl}$ ) is a common way to remove organic matter (Hoenig \& de Kersabiec, 1996; Hoenig, 2001, 2003). However, the general procedure requires the use of a high amount of solid sample (0.2-2 g) (Hoenig, 2003; Soylak et al., 2004). 
Dry ashing in muffle furnace consists of subjecting the solid sample located in a suitable vessel (usually a porcelain crucible) to controlled high temperature $\left(450^{\circ} \mathrm{C}\right)$ until constant weight of the sample is obtained (1-48 h). Usually, these procedures are performed at atmospheric pressure in programmable muffle furnaces. After ashing, the resulting inorganic residue (ash) is dissolved with mineral acids (Hoenig \& de Kersabiec, 1996; Heonig, 2001).

Conventional dry ashing leads to complete removal of the organic matrix and to accurate analytical results for commonly determined elements (Hoenig, 2003). It ensures the quantitative decomposition and elimination of organic matter and an efficient release of elements initially associated with it. Due to the high temperatures involved in the procedure, there are associated risks of loss of elements by volatilization. The elements with highest loss risks are $\mathrm{Se}, \mathrm{As}, \mathrm{Sb}, \mathrm{Ge}, \mathrm{Hg}, \mathrm{Cd}, \mathrm{Pb}$ and $\mathrm{Zn}$. In addition to volatilization problems, other drawbacks could be the insolubilization that occurs when several elements are associated with the silica matrix. Thus, the addition of HF may be required to solubilize the residue (Hoenig \& de Kersabiec, 1996; Hoenig, 2001).

In addition to conventional dry ashing in a muffle furnace, other systems are available. While dry ashing and low temperature ashing (LTA) are carried out in open vessels, combustion bomb, oxygen flask (Schöniger flask) and microwave-induced combustion (MIC) are performed in closed systems. Flores et al. (2007) reviewed sample preparation techniques based on combustion reactions in closed vessels.

In combustion bombs made of stainless steel, a sample mass higher than $0.5 \mathrm{~g}$ is prepared as a pellet and positioned in a metallic cup. A volume of absorbing solution $(5-10 \mathrm{~mL})$ is added to the vessel. Then, the system is closed, pressurized with oxygen and ignition takes place.

A miniaturized approach derived from the previous one consists of the use of a Schöniger flask that employs a small amount of sample (MacDonald, 1961). It is a very classical approach developed in 1955 by W. Schöniger as a pioneer for microanalysis (Schöniger, 1955; Schöniger, 1956). The combustion of the sample takes place in a closed Pyrex conical flask filled with oxygen for approximately $20 \mathrm{~min}$. The sample (3-10 mg) is wrapped in a piece of paper, linked to a Pt wire, and burnt in the flask containing the appropriate absorption solution $\left(\mathrm{H}_{2} \mathrm{O}, \mathrm{H}_{2} \mathrm{O}_{2}, \mathrm{NaOH}\right)$. This strategy is more commonly employed for non-metals and metalloids. The two main limitations observed for this approach are incomplete oxidation and incomplete absorption by the solution (MacDonald, 1971). Usually, a sample with mass smaller than $100 \mathrm{mg}$ is burned in glass vessels of up to $1 \mathrm{~L}$, but using a volume of reagents in the range of 1-20 mL. Other applications of the oxygen flask were applied for determination of Se (Sun \& Li, 2000) and As (Puttermans et al., 1983) in biological tissues (hair, kidney and liver tissue) using an amount of sample in the range of 40-200 mg. Oxygen flask combustion offers a faster and simpler method than $\mathrm{AD}$ in the case of Se and does not suffer from losses of Se.

MIC combines the advantages of microwave-assisted digestion (MAD) and dry ashing. MIC involves the combustion of organic samples in closed quartz vessels 
containing pressurized oxygen but using microwave radiation in the ignition step (Flores, 2007). After combustion, the gases are absorbed in a solution of diluted acid. In MIC, a quartz device is inserted into a quartz vessel and it acts as a sample holder. The sample (less than $0.5 \mathrm{~g}$ ) is prepared as a pellet and temperatures of $1300{ }^{\circ} \mathrm{C}$ are achieved. MIC applications were developed for samples which were difficult to digest by MAD, such as coal (Antes et al., 2010) and biological samples (Duarte et al., 2009; Mesko et al., 2010). Quantification is usually achieved by ICP-MS, or ICP-OES. A recent application of MIC was employed for seafood digestion and As determination by hydride generation coupled to atomic spectrometry (HG-AAS, HG-ICP-MS and ICP-MS) (Duarte et al., 2009). Pellets of sample mass between 100-500 mg and $6 \mathrm{~mL}$ of $0.1 \mathrm{~mol} \mathrm{~L}^{-1} \mathrm{HNO}_{3}$ as the absorbing solution were used. Up to eight samples could be processed simultaneously in $25 \mathrm{~min}$. The residual carbon content was very low $(<0.5 \%)$.

Another possibility is the use of oxygen plasma ashing (i.e., CPA, LTA) which is a low temperature $\left(100{ }^{\circ} \mathrm{C}\right)$ and low pressure (100 Pa) ashing techniques employing a cold plasma of activated oxygen (Hoenig \& de Kersabiec, 1996; Flores, 2007). The sample is contained in a quartz test tube equipped with a cooling finger and the oxygen plasma is generated at high frequency. Volatile elements are retained on the cooling finger during the ashing procedure, avoiding element losses. The temperature reaches $150{ }^{\circ} \mathrm{C}$ and the required volume of acid is only 1-2 mL. However, this method is unsuitable for routine analysis due to the long mineralization time. CPA tends to be slow, taking several hours per sample, for instance, $1.5 \mathrm{~h}$ for muscle tissue, fat, fecal matter filter paper and rat tissue (Gleit \& Holland, 1962), whereas 12-18 h for dentin samples, $48 \mathrm{~h}$ for bones, or even $60 \mathrm{~h}$ for complete destruction of organic matter in non-powdered samples (Sansoni \& Panday, 1994).

Several miniaturized procedures of CPA were applied prior to TXRF analysis in two ways. First, deposition of the sample (a few $\mathrm{mg}$ ) onto the quartz sample carrier after ashing (Reus, 1991; Schemling et al., 1997) and secondly, application of ashing directly to the quartz sample carrier of TXRF (Theisen \& Niessner, 1999). The last option is of interest from the point of view of miniaturization and is called in situ CPA (De la Calle et al., 2013b). Moreover, AD or dry ashing were combined with in situ CPA (Woelfl et al., 2003, 2004; Mages et al., 2008; Wagner \& Mages, 2010; Savoly et al., 2012). In these examples, $2 \mathrm{~h}$ of CPA was required for nematodes and microcrustaceans (Woelfl, 2003; Savoly et al., 2012), and between 1 and $7 \mathrm{~h}$ for filters (Wagner and Mages, 2010).

\subsection{Fusion}

Some samples such as ceramics, aluminosilicates and alloys are difficult to decompose by wet digestion. Fusion offers an alternative for these type of samples. For fusion procedures, basic $\left(\mathrm{NaOH}, \mathrm{Na}_{2} \mathrm{CO}_{3}\right)$, acidic $\left(\mathrm{K}_{2} \mathrm{~S}_{2} \mathrm{O}_{7}\right)$, oxidizing $\left(\mathrm{Na}_{2} \mathrm{O}_{2}\right)$ and reducing (carbide) fluxes are used. Both powdered samples and fluxes are mixed in a crucible and this mixture is heated to a temperature above the melting point of the 
flux in a furnace or burner and agitated until total dissolution occurs (Claisse, 2003). Approximate proportions of sample/flux are $1 \mathrm{~g}$ of sample per $10 \mathrm{~g}$ of flux. Fusion processes usually last between $1-3 \mathrm{~h}$ at a temperature in the range of $300-1000{ }^{\circ} \mathrm{C}$. The high concentration of added fluxes and the aggressive media increase the risks of contamination and the amount of added salts may produce matrix interferences. In addition, the loss of volatile components has to be carefully controlled.

Based on a large scale procedure using $0.5 \mathrm{~g}$ of sample and $2 \mathrm{~g}$ of flux $\left(\mathrm{Na}_{2} \mathrm{CO}_{3}\right.$ : $\mathrm{ZnO}, 1: 1 \mathrm{~m} / \mathrm{m}$ ) loaded into a platinum crucible and heated in a microwave muffle furnace for $1 \mathrm{~h}$ (including cooling time), a miniaturized procedure was developed (Associaçao Brasileira de Cimento Portland, 2003). In situ fusion on the boat-type graphite platform for the direct determination of elements (Cr, Co and $\mathrm{Mn}$ ) by ETAAS in cement samples by solid sampling was proposed as a miniaturized approach (Intima \& Väisänen, 2009). This strategy works with up to $200 \mu \mathrm{g}$ of sample. It consists of using $10 \mu \mathrm{L}$ of a flux mixture (consisting of $4 \%(\mathrm{~m} / \mathrm{v}) \mathrm{Na}_{2} \mathrm{CO}_{3}+4 \%(\mathrm{~m} / \mathrm{v}) \mathrm{ZnO}$ $+0.1 \%(\mathrm{~m} / \mathrm{v})$ Triton X-100) added over the cement sample and heated at $800{ }^{\circ} \mathrm{C}$ for $20 \mathrm{~s}$ in the ETAAS platform. The addition of Triton X-100 was necessary to facilitate the interaction between the flux and the sample. After the fusion step, the heating is stopped, the platform is removed and an aliquot of $10 \mu \mathrm{L}$ of $0.1 \%(\mathrm{~m} / \mathrm{v}) \mathrm{HNO}_{3}$ is added to the resulting mold which has been completely dissolved. Then, the platform was re-introduced into the graphite tube and the heating program was continued to the end. This method is faster than the normal-scale fusion and allows direct determination on the boat-type graphite platform (Intima \& Väisänen, 2009).

\subsection{Acid Digestion and Microwave-assisted Digestion}

Conventional AD, also called wet digestion, involves the total dissolution of the sample matrix, which ensures the complete availability of analytes for the analysis (Mitra \& Brukh, 2003). This is probably the most common sample preparation procedure for solid samples.

Conventional $\mathrm{AD}$ generally encompasses the use of acids $\left(\mathrm{HNO}_{3}, \mathrm{HCl}, \mathrm{H}_{2} \mathrm{SO}_{4}\right.$, or $\mathrm{HF}$ ) and oxidants $\left(\mathrm{H}_{2} \mathrm{O}_{2}\right)$. Furthermore, traditional heating systems such as a sand bath, hot plate, furnace, or Bunsen burner are used for AD (Bendicho et al., 2011). The simplest configuration consists of placing the sample and the acids in a glass beaker covered with a watch glass while heating. When the sample is digested, the acid is evaporated to near dryness and diluted in acid solution (Mitra \& Brukh, 2003). Conventional AD generally uses a large amount of sample (0.3-5 g) and 10-100 mL of concentrated reagents while the heating is performed at elevated temperatures depending on the employed acids $\left(120-330^{\circ} \mathrm{C}\right)$ for long periods of time (4-48 $\mathrm{h}$ ) (Soylak et al., 2004).

Faster and more focused digestion was developed using microwave energy since 1975 (Abu-Samra et al., 1975), giving rise to MAD. The principles, equipment and applications of microwave-assisted techniques have been described in book chapters (Luque de Castro \& Luque-García, 2002; Matusiewicz, 2003) and many review articles 
(Smith \& Arsenaut, 1996; Luque-García \& Luque de Castro, 2003). This approach has been widely applied and established as a reference sample preparation procedure for trace element analysis in routine analysis and research laboratories. There are different schemes for AD methods (Matusiewicz, 2003), including open systems (conventional heating, microwave heating and ultraviolet digestion), closed systems (conventional heating, microwave heating), and flow systems (conventional heating, UV on-line decomposition and microwave heating). Recently, a combined procedure of exposure to infrared radiation (IR) for $10 \mathrm{~min}$ followed by cavity microwave-assisted digestion was proposed for the digestion of a food complement which includes flaxseed, wheat bran, wheat germ, cashew nuts, soybeans, sesame seeds, brown sugar and oats (Dantas et al., 2013). Moreover, several pieces of equipment were developed to improve its safety and efficiency. Both low and high pressure digestion can be employed in MAD. In addition, focused-microwave and cavity-microwave digestion have also been reported (De Oliveira, 2003).

MAD reduces the time of operation from 1-2 $\mathrm{h}$ in conventional AD to 5-15 min (Matusiewicz, 2003). Conventional MAD requires 50-120 mL vessels, the addition of 5-10 mL of reagents (usually acids and $\mathrm{H}_{2} \mathrm{O}_{2}$ ) and a sample mass of up to $0.5 \mathrm{~g}$ (Soylak et al., 2004; Borgese et al., 2009).

In order to reduce the amount of sample and necessary reagents, several miniaturized strategies have been tested by researchers using electric ovens, hot plates, domestic microwave ovens, microwave systems, focused-microwaves and cavitymicrowaves (Luque-García \& Luque de Castro, 2003).

One possibility of miniaturization is the use of $7 \mathrm{~mL}$ vials as sample holders and carrying out the sample digestion by means of an electric oven (W. Zhang et al., 2012) and/or a hot plate (Yu et al., 2001; W. Zhang et al., 2012). As an example, sample mass ranges from 50 to $100 \mathrm{mg}$ of geological material and $4.5 \mathrm{~mL}$ of digestion reagents $\left(\mathrm{NH}_{4} \mathrm{HF}_{2}\right.$ (W. Zhang et al., 2012) and $\mathrm{HNO}_{3}+\mathrm{HF}$ (Yu et al., 2001)) have been reported. Similarly, $2 \mathrm{~mL}$ polypropylene vials were used for the digestion of hair samples and bovine liver ( $15 \mathrm{mg})$ using a conduction oven $\left(20 \mathrm{~h}\right.$ at $\left.70{ }^{\circ} \mathrm{C}\right)$ or a conventional microwave oven $\left(7 \mathrm{~min}\right.$ ). Only $50 \mu \mathrm{L}$ of $\mathrm{H}_{2} \mathrm{SO}_{4}+150 \mu \mathrm{L}$ of $\mathrm{HNO}_{3}$ were needed for the digestion (De Oliveira, 2003; Flores, 2007). Another recent option consists of using a domestic microwave oven for the partial digestion of bovine tissue prior to ICP-OES (Matos et al., 2009). In this case, a sample mass of $50 \mathrm{mg}$ was placed in a $4 \mathrm{~mL}$ laboratorymade Polytetrafluoroethylene (PTFE) microvessel inside a baby-bottle sterilizer. Very low volumes of $\mathrm{HNO}_{3}(50 \mu \mathrm{L})$ and $\mathrm{H}_{2} \mathrm{O}_{2}(100 \mu \mathrm{L})$ were added to the uncovered microvessels and positioned inside the sterilizer, containing $500 \mathrm{~mL}$ of water and exposed to microwave radiation for $7 \mathrm{~min}$ (Matos et al., 2009).

More attention has been paid to the miniaturization of MAD approaches since the mid-1990s (Baldwin et al., 1994) by using microwave energy and significantly reducing the amount of sample (0.5 mg - $0.1 \mathrm{~g})$ and the volume of acids (0.1-1 mL). These approaches are commonly named small-volume microwave-assisted digestion or LVMAD. 
First, smaller vessels (20 mL) were developed for the analysis of organic and biological microsamples (Müller, 1998). These modified vessels allow digestion of 50-100 mg of sample using only $0.7 \mathrm{~mL}$ of $\mathrm{HNO}_{3}$ (Müller, 1998). Moreover, $15 \mathrm{~mL}$ polystyrene vessel liners were used for analysis of $2-10 \mathrm{mg}$ of biopsy samples after adding $0.5 \mathrm{~mL}$ of $\mathrm{HNO}_{3}$. However, specific microwave ovens and rotors, which are not available in all labs, are needed in this approach (Bocca et al., 2007). Several examples of small-volume MAD can be found for the analysis of different elements combined with FAAS (Baldwin et al., 1994), ETAAS (Deaker \& Maher, 1997, 1999; Iavicoli et al., 2001), TXRF (Varga et al., 2005), HG-AFS (Zhao et al., 2010), ICP-OES (Millos et al., 2009) and ICP-MS (Esslemont et al., 2000; Maher et al., 2001; Varga et al., 2005; Millos et al., 2008). Diverse configurations were developed in the literature for small-volume MAD with the goal of increasing the sample throughput: i) 1 vial of $7 \mathrm{~mL}$ (Maher et al., 2001; Iavicoli et al., 2001; Varga et al., 2005), ii) 2 vials of $7 \mathrm{~mL}$ placed one over the other (Baldwin et al., 1994; Deaker \& Maher, 1997, 1999; Esslemont et al., 2000), iii) 3 vials of $6 \mathrm{~mL}$ placed one over the other (Figure 7.5B) (Millos et al., 2008, 2009), iv) 3 vials of $6 \mathrm{~mL}$ positioned in triangle (Zhao et al., 2010) or v) 4 vials of $2 \mathrm{~mL}$-polypropylene minivials positioned in circle (Brancalion et al., 2005), but all placed in larger PTFE vessels of 100-120 mL. As can be noted, high throughput can be achieved when multiple small vials are placed inside a large PTFE vessel, and placed in a rotor of 6-12 digestion vessels.

Moreover, miniaturization was also extended to focused-MW (FMW). In one study, 4 vials of $4 \mathrm{~mL}$ or 5 vials of $2 \mathrm{~mL}$ were placed inside a glass tube of a FMW for the determination of metals in hair samples (Tan, 2001; De Oliveira, 2003). The mass of sample employed was in the range of 30-60 mg. Similarly, 4 polypropylene minivials of $2 \mathrm{~mL}$ positioned in circle were placed in a support fitted in a glass vessel for the digestion of medicinal plants and determination of Cd (Brancalion et al., 2005). The digestion of $5 \mathrm{mg}$ of sample was carried out by adding $0.2 \mathrm{~mL}$ of $\mathrm{HNO}_{3}+0.15 \mathrm{~mL} 30 \%$ (v/v) $\mathrm{H}_{2} \mathrm{O}_{2}$ for 4-10 min.

There is even a more miniaturized approach that it is carried out directly for a specific analytical technique called TXRF due to the type, form and configuration of the sample carrier that is used. The sample carrier in TXRF is generally a disc of quartz with a diameter of $30 \mathrm{~mm}$ and a thickness of $3 \mathrm{~mm}$ (De la Calle et al., 2013b). These miniaturized sample preparation procedures are called in situ AD and in situ MAD, depending on the heating source, usually a hot plate (Woelfl et al., 2003; Sávoly et al., 2012) or a domestic microwave oven (Marcó et al., 2001). These approaches consist of placing a very small amount of sample ( $\mu \mathrm{g}-\mathrm{mg}$ ) directly onto the sample carrier and adding a very small volume of reagents $\left(5-20 \mu \mathrm{L}\right.$ of $\mathrm{HNO}_{3}$ or $\left.\mathrm{HNO}_{3}+\mathrm{H}_{2} \mathrm{O}_{2}\right)$. Then, the digestion occurs at $90-100{ }^{\circ} \mathrm{C}$ over 10 min using a hot plate (Woelfl et al., 2003; Sávoly et al., 2012) and 8 min using a domestic microwave oven (Marcó et al., 2001). Several examples of this approach are reported in the literature for the analysis of nematodes (Sávoly et al., 2012), freshwater microcrustaceans, (Woelfl et al., 2003; Mages et al., 2004), fish embryos (Mages et al., 2008), bovine liver tissue (Marcó et al., 2001) and human hair (Borgese et al., 2010). 


\subsection{Vapor-phase Acid Digestion and Vapor-phase Microwave-assisted Digestion}

VPAD is an alternative to AD in which the attack of the sample occurs from the acidvapor phase rather than the solution. The most important advantages of VPAD are the decreased acid concentration of the digestates, the lower dilution and, as a consequence, the improvement of detection limits (Matusiewicz, 1991; Matusiewicz et al., 1991). Moreover, this approach allows the simultaneous purification of the acid, prevents the introduction of impurities and improves blank samples (Araújo et al., 2004; Matos et al., 2009). This procedure has proved useful for biological tissues since both organic and inorganic components are completely solubilized by vapor phase attack (Matusiewicz et al., 1991).

Vapor-phase digestion has been employed for sample preparation using different inorganic acids by conventional heating (Thomas \& Smythe, 1973; Klitenick et al., 1983), PTFE pressure bombs (Czégény et al., 1998; Matusiewicz, 1989), a high-pressure asher (Amarasiriwardena et al., 1994) and a high-pressure digestion vessel (Lásztity et al., 1995).

Furthermore, microwave energy was employed for accelerating VPAD, giving rise to VPMAD using closed-vessel microwave systems (Matusiewicz et al., 1991), and, more recently, FMW ovens (Araújo et al., 2000; Trevizan et al., 2003; Araújo et al., 2004).

In 1991, Matusiewicz et al. developed VPMAD using a laboratory-made high pressure-digestion bomb (Matusiewicz et al., 1991). A PTFE microsampling cup with one leg (to lift the vial up off the reagents) was used to place the sample (250 $\mathrm{mg}$ ) and $0.6 \mathrm{~mL}$ of acid was added to the vial. Then, the unit was placed inside a $100 \mathrm{~mL}$ perfluoroalkoxy-PTFE vessel containing $6 \mathrm{~mL}$ of acids which are not in direct contact with the sample and thus only the acid vapors are able to digest the sample.

VPAD and VPMAD can be considered miniaturized approaches since very small amounts of sample are generally used. Usually, a small amount of sample is used (less than $250 \mathrm{mg}$ ) and a small volume of $\mathrm{H}_{2} \mathrm{O}_{2}$ or $\mathrm{HNO}_{3}(150-600 \mu \mathrm{L})$ is added to the sample to prevent charring of organic matter (Matusiewicz et al., 1991). Usually, micro-vessels are employed for sample digestion of small masses (Matusiewicz et al., 1991; Araújo et al., 2004), which are advantageous in these procedures because they improve the interaction of the vapor with sample particles (Araújo et al., 2004; Matos et al., 2009). Moreover, this characteristic is attractive when the sample size is limited, such as in the case of toxicological analysis of biological tissues. Since then, different configurations were developed in order to increase the sample throughput (Araújo et al., 2000; Eilola \& Perämäki, 2001).

A VPMAD was developed for the digestion of organic samples (polymers, biological and drug samples) using quartz inserts inside the digestion vessels (3-12 vessels) placed in a carrousel in a microwave oven (Eilola \& Perämäki, 2001, 2003, 2009; Niemelä et al., 2004). The sample mass was in the range of 0.1-0.2 g (Eilola \& Perämäki, 2001, 2003; Niemelä et al., 2004) and 0.5 g (Eilola \& Perämäki, 2009). Two sample 
digestion arrangements were used, consisting of placing a quartz insert without the holder or a quartz insert with a three-legged holder inside a digestion vessel. A holder was employed in order to segregate the sample vial away from the reagents, since conductive heating could enhance the digestion if hot acids are in contact with the insert wall. Several modifications were made in a similar system depending on the difficulty of sample digestion:

1. Two-step procedure VPMAD+MAD (Eilola \& Perämäki, 2001). First, in the VPMAD phase the sample was placed in the quartz insert (without direct contact with the

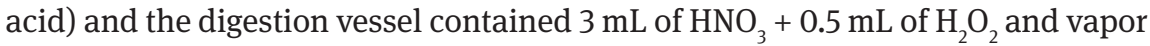
phase digestion took place for $80 \mathrm{~min}$. After that, since a small residue remained, $5 \mathrm{~mL}$ of diluted $\mathrm{HNO}_{3}$ or $\mathrm{HF}$ were added to the sample which was digested for $15 \mathrm{~min}$ in a microwave oven.

2. Simultaneous VPMAD and MAD (Eilola \& Perämäki, 2003; Niemelä et al., 2004). The sample was placed in the quartz insert and $1 \mathrm{~mL}$ of $\mathrm{H}_{2} \mathrm{SO}_{4}+0.5 \mathrm{~mL}$ of $\mathrm{HNO}_{3}$ was directly added to the sample and the digestion vessel contained $3 \mathrm{~mL}$ of $\mathrm{HNO}_{3}$ (+ $0.5 \mathrm{~mL}$ of $\mathrm{H}_{2} \mathrm{O}_{2}$ ). Two MW programs of heating were performed while allowing the vials to cool down between programs.

3. VPMAD using a quartz insert and a holding insert (Eilola \& Perämäki, 2009). The sample was placed in the quartz insert (without direct contact with the acid),

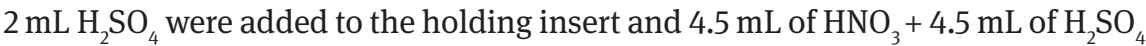
were added to the digestion vessel. A MW program of heating was performed and, in the case where a solid residue remained, $5 \mathrm{~mL}$ of hot $\mathrm{HNO}_{3}$ was added to complete digestion.

In the case of FMW, a homemade PTFE support was designed by placing 4 cups containing the sample $(30 \mathrm{mg}$ ) one over another at different heights from the base of the support. Then, the support was adapted to fit on the microwave glass vessel of FMW containing $15 \mathrm{~mL}$ of $\mathrm{HNO}_{3}$ in the bottom part. As a consequence of MW heating, the acid vapor partially condensed in the upper part of the flask and was partially collected in each sample cup, thus the samples were directly exposed to acid steam (Figure 7.5C) (Araújo et al., 2000, 2004). This procedure was applied for the determination of Fe and Co from biological samples by ETAAS. It was observed that the distance of the sample cup to the support is a critical parameter.

In a similar way, a VPMAD procedure was adapted for TXRF analysis (Varga et al., 1999). In this procedure, freeze-dried algae samples (6-21 mg) were placed in $2 \mathrm{~mL}$ open quartz microvials and $75 \mu \mathrm{L}$ of $30 \%(\mathrm{~m} / \mathrm{v}) \mathrm{HNO}_{3}$ was added to the microvials. The vials were placed in $120 \mathrm{~mL}$ PTFE microwave vessels and the vapor-digestion took place for $90 \mathrm{~min}$ at $180{ }^{\circ} \mathrm{C}$ (Varga et al., 1999).

Likewise, VPMAD was extended for the direct analysis of cancer cells in the TXRF sample carrier for the determination of $\mathrm{Cu}$, Fe and $\mathrm{Zn}$ (Figure 7.5D) (Szosboszlai et al., 2008). Cancer cells were placed directly into the quartz sample carrier. Then, it was covered with a cap and assembled onto a three-leg glass support inside the digestion 
vessel. Finally, $3 \mathrm{~mL}$ of $\mathrm{HNO}_{3}$ were added to the digestion vessel and the $\mathrm{HNO}_{3}$ vapor attacked and digested the cancer cell sample. However, it should be noted that in this approach of vapor phase digestion developed for TXRF, higher concentrations of organic and inorganic matter remained in the sample than in conventional AD or MAD due to incomplete decomposition.

\subsection{Ultrasound-assisted Digestion and Pseudodigestion}

Another possibility for AD is the application of ultrasound energy, which gives rise to ultrasound-assisted digestion (UAD). UAD encompasses total or partial matrix decomposition (digestion or pseudodigestion). UAD only provides information of the total or pseudo-total metal content present in the solid sample. Generally, concentrated acids and oxidants are used, even HF which is used to solubilize the silicate matrix. When HF is employed, mostly in UAD but also in UAE (discussed in section 7.4.1.3.2.), the use of ultrasonic probe should be avoided because of damage to the probe tip (De la Calle et al., 2009). As a consequence, higher sonication times are usually applied, from $1 \mathrm{~min}$ up to $2 \mathrm{~h}$ in an ultrasonic bath, depending on the type of sample (e.g., 1-5 min for biological tissue samples (Arain et al., 2007), 5-10 min for plant tissue (Kazi et al., 2009a) and 18-30 min for soils and sediments (Brunori et al., 2004; Ilander \& Väisänen, 2007, 2009; Pontes et al., 2010).

Several applications of UAD using the ultrasonic bath were reported for a wide variety of samples, including muscle tissue (Arain et al., 2007), cigarette (Kazi et al., 2009a), sediments (Brunori et al., 2004), street dust samples (Elik, 2005) and ash (Ilander \& Väisänen, 2007, 2009; Pontes et al., 2009). Typical mixtures include only $\mathrm{HNO}_{3}$ (Kazi et al., 2009a), $\mathrm{HNO}_{3}: \mathrm{H}_{2} \mathrm{O}_{2}$ (1:1, v/v) (Brunori et al., 2004; Arain et al., 2007), $\mathrm{HNO}_{3}: \mathrm{HClO}_{4}: \mathrm{HF}(2: 1: 1, \mathrm{v} / \mathrm{v} / \mathrm{v})$ (Elik, 2005), or aqua regia $+\mathrm{HF}$ (Ilander \& Väisänen, 2007, 2009; Pontes et al., 2009). Variable volumes of reagents can be employed in an ultrasonic bath, usually ranging from 1-10 mL (Ilander \& Väisänen, 2007, 2009; Kazi et al., 2009b). However, there is a growing trend toward the reduction of the volume of reagents and mass of sample. For instance, Kazi et al. (2009b) employed an ultrasonic bath for the UAD of heavy metals (Cd, Cr, Ni and $\mathrm{Pb}$ ) from biological and environmental samples. They used a mass of sample of $0.1 \mathrm{~g}$ and $1 \mathrm{~mL}$ of extractant phase. The digestion reagent composition and the sonication time depended on the type of sample. Thus, sonication times of 2-20 min and a mixture of $\mathrm{HNO}_{3}: \mathrm{H}_{2} \mathrm{O}_{2}(2: 1, \mathrm{v} / \mathrm{v})$ and $\mathrm{HNO}_{3}$ were used for biological samples, while a sonication time of $5-50 \mathrm{~min}$ and a mixture of concentrated $\mathrm{HNO}_{3}: \mathrm{HClO}_{4}: \mathrm{HF}$ (2:1:1, v/v/v), $\mathrm{HNO}_{3}: \mathrm{HCl}(1: 3, \mathrm{v} / \mathrm{v}), \mathrm{HNO}_{3}: \mathrm{H}_{2} \mathrm{O}_{2}(2: 1, \mathrm{v} / \mathrm{v})$ and $\mathrm{HNO}_{3}$ was used for soils and sediments.

In the case of UAD, the cup-horn sonoreactor has not yet been used. Also, it is sometimes difficult to distinguish between UAD and UAE with the different ultrasonic devices since partial digestion of the sample occurs in both techniques (De la Calle et al., 2009, 2011a). However, the main differences are that more dilute acids and shorter sonication times are employed in UAE. 


\subsection{Enzymatic digestion}

Enzymatic digestion is mainly used for drug analysis and is less used for matrix degradation prior to total trace element analysis and chemical speciation due to the long operation time and low analyte recovery (Bermejo et al., 2004). Generally, hydrolytic enzymes (i.e., lipases, amylases, proteases) are used. These enzymes catalyze the introduction of water at a specific bond of the substrate (Bermejo et al., 2004), which provokes protein destruction and tissue degradation (Carpenter, 1981) and, as a consequence, metals associated with hydrolyzable proteins are released to the aqueous medium (Bermejo et al., 1999). Moreover, using hydrolytic enzymes it is possible to differentiate between fractions of elements associated to diverse components of the sample matrix, maintaining the chemical form of a species after the enzymatic digestion and thus making speciation analysis possible (Bermejo et al., 1999). The procedure for total metal analysis by enzymatic digestion usually involves the mixture of the sample with the enzyme and a buffer medium and incubation at $37-55^{\circ} \mathrm{C}$ for a certain time, which usually ranges from 4 to $48 \mathrm{~h}$.

In 1981, Carpenter published the first work on proteolytic enzymes for the digestion of kidney and liver tissue and the determination of $\mathrm{Cd}, \mathrm{Cu}, \mathrm{Pb}$ and $\mathrm{Tl}$ by FAAS (Carpenter, 1981). In this work, a very high amount of tissue (5 g) was used, and crystalline subtilisin A (5 mg) was employed as a non-specific protease (a protein-digesting enzyme). The tissue and the enzyme were mixed in $30 \mathrm{~mL}$ of buffer and incubated at $55^{\circ} \mathrm{C}$ for $60 \mathrm{~min}$.

In addition to biological tissues (Tan \& Marshall, 1997; Chen \& Marshall, 1999; Bermejo et al., 1999), enzymatic digestion was also applied to sediments (Turner \& Olsen, 2000; Turner et al., 2001; Turner, 2006). In these examples, and as a first step towards miniaturization, a sample mass lower than that employed by Carpenter $(0.2 \mathrm{~g})$ and a lower volume of buffer $(3-10 \mathrm{~mL})$ were used. The samples were incubated for $4-8 \mathrm{~h}$ at $37^{\circ} \mathrm{C}$ in presence of different enzymes. In some papers, the enzymatic digestion was accelerated by using high-pressure homogenization, which applied gentle agitation prior to introducing the slurry into the instrumentation (Tan \& Marshall, 1997; Chen \& Marshall, 1999).

As a second step toward minaturization, these procedures were accelerated by the use of ultrasound energy, giving rise to ultrasound-assisted enzymatic digestion (Vale et al., 2008). Both ultrasonic baths (Peña-Farfal et al., 2004) and probes (Capelo et al., 2004; Cabañero et al., 2005; Maduro et al., 2006; Vale et al., 2007) were used in order to speed up enzymatic digestion. Although different publications addressed the increase of enzymatic kinetics under ultrasound energy (Capelo et al., 2004), its effects are still controversial. Some authors confirm that cavitation boosts enzymesubstrate kinetics (Bermejo et al., 2004; Vale et al., 2008), while others maintain that denaturation of the enzymes took place and, as a consequence, their activity declined (Talukder et al., 2006). In the case of using an ultrasonic bath, the incubation time was reduced to $30 \mathrm{~min}$ when sonicated (Peña-Farfal et al., 2004), but similar mass sample and volume of reagents to the previously mentioned publications $(0.2 \mathrm{~g}$ of 
sample, $7 \mathrm{~mL}$ of buffer and $20 \mathrm{mg}$ of enzyme) were employed. On the contrary, the EPS approach is more appealing since the operation time was reduced to just a few seconds (Capelo et al., 2004; Maduro et al., 2006; Vale et al., 2007). The amount of sample used in these cases was reduced to $10 \mathrm{mg}$, the amount of enzyme was in the range of $1-10 \mathrm{mg}$ and the volume of water or buffer was $1 \mathrm{~mL}$. The sonication time varied from $5 \mathrm{~s}$ in the case of Se in yeast (Capelo et al., 2004), to 15-300 s for Cd and Pb in BCR-101 spruce needles, BCR-414 plankton, BCR-679 white cabbage, BCR-710 oyster tissue and IAEA-0392 algae (Maduro et al., 2006), and $120 \mathrm{~s}$ for Se in oyster tissue, BCR-414 plankton and ERM-CE 278 mussel tissue (Vale, 2007).

Although enzymatic digestion does not compete with conventional approaches for trace metal analysis from the point of view of operation time, these procedures are generally carried out under mild conditions of temperature and pressure due to the temperature-dependent activity of enzymes. As a result, there are low losses by volatilization and reduced risks of contamination (Bermejo et al., 2004). When using ultrasound power, this methodology meet the requirements of miniaturization, because the reactions are generally carried out in Eppendorf vials of $1 \mathrm{~mL}$ with low amount of samples (10 mg) and in a short period of time (few seconds). Moreover, this procedure offers low cost, low sample requirements and low generation of wastes (Bermejo et al., 2004).

Enzymes have also been involved in sample pre-treatment for speciation analysis (Vale et al., 2008; Mesko et al., 2011). Several examples were found for Se speciation (organic and inorganic species) in animal tissue and oyster (Quijano et al., 2000; Moreno et al., 2001). These conventional approaches required $24 \mathrm{~h}$ of enzymesample incubation. Similar approaches were accelerated by reducing the incubation time in presence of ultrasonic waves to only 1-15 min. EPS applications were reported for Se speciation in plants (Montes-Bayón et al., 2006) and krills (Siwek et al., 2006) and As speciation in rice and chicken samples (Sanz et al., 2005a, 2005b). However, the most miniaturized and accelerated approaches for speciation involve the use of lower amounts of sample (around $10 \mathrm{mg}$ instead of $0.2-3 \mathrm{~g}$ ) and reduced sonication time (around 0.5-10 min). Some examples of miniaturized approaches were found for Se speciation in plants (Pedrero et al., 2007), yeast, oyster tissue and mussel tissue (Capelo et al., 2004), and As speciation in human hair (Sanz et al., 2007).

\subsection{Tissue Solubilization}

In the case of solubilization procedures, the matrix is not significantly altered but completely dissolved (i.e., the solid sample is converted into a liquid). These procedures can be divided according to the reagent used: buffer salts, surfactants, reducing agents, TMAH, and acids (Mesko et al., 2011).

Buffer salts, such as tris(hydroxymethyl)aminomethane (Tris), are commonly used to obtain the necessary ionic strength for protein solubilization of the sample. Tris buffer can be used in combination with $\mathrm{HCl}$ so that the free and weakly bound analyte species or metallo-amino acids are released (Mesko et al., 2011). The general 
trend toward miniaturization lies on the reduction of volumes $(1 \mathrm{~mL})$ and sample masses (20 mg) (Tran et al., 2010; Lavilla et al., 2012) and the speeding up of the procedure by applying ultrasound energy (3 min) (Lavilla et al., 2012).

Surfactants such as sodium dodecyl sulfate (SDS) are recommended for element speciation. SDS provokes the disruption of the cell membranes, breaking the interactions between lipids and proteins. As a consequence, the metal-binding proteins are solubilized and hydrophobic interactions are prevented (Szpunar et al., 2003; Mesko et al., 2011). As a miniaturized approach, $20 \mathrm{mg}$ of fungi separated from maize sample was solubilized using $1 \mathrm{~mL}$ of Tris- $\mathrm{HCl}$ containing SDS and boiling in a water bath for 5 min (Muñoz et al., 2005a).

TMAH is a strong alkaline reagent that helps tissue solubilization. TMAH provokes hydrolytic scission, methylation and breaks sulfide chemical bonds in proteins (Nóbrega et al., 2006). The use of TMAH was also reported for Hg and As speciation (Sanz et al., 2007; Reyes et al., 2008). Some of the drawbacks of TMAH solubilization include limited time-stability of the obtained solution and the prolonged treatment time that is necessary (Subramanian, 1996). Conventional approaches involved the solubilization of $0.1-1 \mathrm{~g}$ of sample in $1-5 \mathrm{~mL}$ of $25 \%(\mathrm{~m} / \mathrm{v})$ TMAH over $8-48 \mathrm{~h}$ at room temperature or $2-8 \mathrm{~h}$ at $50-70{ }^{\circ} \mathrm{C}$ (Subramanian, 1996). Reduction of volume of TMAH solution up to $0.5 \mathrm{~mL}$ with occasional shaking was performed at room temperature for $2 \mathrm{~h}$ for the determination of $\mathrm{As}, \mathrm{Cd}, \mathrm{Ni}$ and $\mathrm{Pb}$ in human hair (Ribeiro et al., 2000). Recently, the solubilization of tissues using TMAH in $\mathrm{MeOH}$ was also accelerated by using ultrasound energy, either by using an ultrasonic bath (Silva et al., 1999; Reyes et al., 2008) (30-45 min) or probe (2-4 min) (Matusiewicz \&Golik, 2004; Sanz et al., 2007). The main advantage of these procedures is the low losses of metals by volatilization. Different techniques were employed for the analysis of samples after treatment with TMAH, such as ICP-OES (Matusiewicz \& Golik, 2004), ICP-MS (Reyes et al., 2008) or ETAAS (Silva et al., 1999).

Ethylenediaminetetraacetic acid (EDTA) in alkaline medium has been used for solubilization of plant tissue in combination with probe-sonication (for only $3 \mathrm{~min}$ ) followed by FAAS (Ca, Fe, Mg, Mn and $\mathrm{Zn}$ ) and ETAAS (Pb and $\mathrm{Cd}$ ) determination (Filgueiras et al., 2001). Moreover, microwave energy was also employed for the acceleration (30 min digestion +10 min cooling) of biological sample solubilization using TMAH in combination with EDTA (Zhou et al., 1996b).

Several reducing agents including dithiothreitol (DTT), dithioerythritol (DTE) and $\beta$-mercaptoethanol were employed with the aim of i) reducing the disulfide bonds, ii) helping protein denaturation, iii) avoiding protein oxidation and iv) improving the solubilization of proteins (Mesko et al., 2011), all of which can assist with tissue solubilization. A volume of about $10 \mathrm{~mL}$ of a $0.01 \%$ DTT solution was used in some applications (Goenaga-Infante et al., 2004, 2005). 


\subsubsection{Extraction-based Techniques}

Extraction is usually a time-consuming step that involves the transfer of analytes of interest from the solid sample to another phase. Solid-liquid extraction (SLE) and MSPD are some options for the analysis of solid samples without previous treatment.

SLE of metals and organometallics from environmental matrices has been commonly called leaching (Camel, 2000). SLE can also be accelerated by means of: i) acids, ii) ultrasound energy, iii) microwave energy, iv) solvents and v) supercritical fluids.

For the extraction of metals from solid samples, the most extended approach is based on acid extraction and accelerated by heating or ultrasound irradiation. MAE and supercritical fluid extraction (SFE) are mostly employed for organic compounds, but several applications to metal speciation and fractionation procedures exist.

\subsection{Acid Extraction (leaching)}

Numerous elements can be extracted from a solid matrix by adding diluted acids under soft conditions. Agitation and temperature can accelerate this process. This scenario is not considered a proper acid digestion because the organic matrix is not destroyed, but dissolution of the rest of the sample and release of the metals does occur. In some case, the metal-matrix bond breaks down without alteration of the matrix. This process is called acid extraction or acid leaching (Welz \& Sperling, 1999).

Diluted acids such as $\mathrm{HNO}_{3}, \mathrm{HCl}, \mathrm{HClO}_{4}, \mathrm{H}_{2} \mathrm{O}_{2}$ or mixtures such as $\mathrm{HNO}_{3}+\mathrm{HCl}$, acetic acid, and even HF (for plant and rock analysis) were used. Leaching could be performed from room temperature to $105^{\circ} \mathrm{C}$ (Welz \& Sperling, 1999). The time necessary to perform the extraction process varies from $5 \mathrm{~min}$ to $24 \mathrm{~h}$. Several representative examples found in the literature used $30 \mathrm{~min}$ for vegetables (Wieteska et al., 1996), $1 \mathrm{~h}$ for mussel tissue (Solchaga et al., 1986), $4 \mathrm{~h}$ for bovine liver (Steiner et al., 1987), $24 \mathrm{~h}$ for eggs or fih tissue (Benson et al., 1983) and overnight for lake sediments (De Vevey et al., 1993). In some of these examples, the approximate sample mass was about $0.5 \mathrm{~g}$ and the volume of acid used was $5 \mathrm{~mL}$.

This procedure avoids the organic matrix destruction step and results in a simple, versatile and inexpensive method with reduced hazards and low risks of loss and contamination. However, it can only be applied to limited matrices and specific elements (Welz \& Sperling, 1999).

Fractionation studies have also been subjected to miniaturization by magnetic agitation extraction (Filgueiras et al., 2002b; De la Calle et al., 2013a). Specifically, they consisted of a scale reduction, but maintained the same mass-to-volume ratio of the operationally-defined protocols of BCR (Quevauviller et al., 1993) and EDTA and acetic acid extractable metal content from soils (Quevauviller et al., 1997). In the case of BCR, the sample mass was reduced from $0.5 \mathrm{~g}$ to $25 \mathrm{mg}$ and the volume of reagents from 5-20 mL to $1 \mathrm{~mL}$ or $250 \mu \mathrm{L}$, respectively (depending on the step). In the case of EDTA and acetic acid extraction, the sample mass was reduced from $5 \mathrm{~g}$ to $0.1 \mathrm{~g}$ for the EDTA-extractable fraction and to $25 \mathrm{mg}$ for the acetic acid-extractable fraction. 
Moreover, the extractant volume was reduced from $50 \mathrm{~mL}$ for extractions with EDTA and $200 \mathrm{~mL}$ for extractions with acetic acid to just $1 \mathrm{~mL}$. However, the same long agitation times in the operationally-defined procedures were used for the BCR ( $48 \mathrm{~h})$ (Filgueiras et al., 2002b), EDTA (1 h) and acetic acid extractions (16 h) (De la Calle et al., 2013a).

\subsection{Ultrasound-assisted Extraction}

Recently, ultrasound energy has been expanded to sample pre-treatment due to the enhancement of diverse processes (Capelo et al., 2005; Santos-Junior et al., 2006; Priego-Capote \& Luque de Castro, 2007; Bendicho et al., 2012a). One of these procedures, UAE, has gained increased attention during recent years due to its simplicity and reliability. UAE was developed in the 1990s, when Miller-Ihli (1989) realized that partial extraction of several elements took place when using USS. The first study involved a sample mass of 3-5 mg and reagent volumes of 2.5-5 mL (Mierzwa et al., 1997), while another used $0.2 \mathrm{~g}$ of sample in $10 \mathrm{~mL}$ of solvent (El-Azouzi et al., 1998). Some discrepancies were found in the sonication time employed and the recoveries achieved by different authors using different ultrasonic devices and types of samples.

Power ultrasound provokes particle disruption and, as a consequence, facilitates penetration of the extraction solvent inside the particles and the release of metals (Mason \& Lorimer, 2002). Ultrasound energy can speed up SLE of elements from different matrices for different purposes, including the determination of total-element contents, speciation analysis and fractionation studies (e.g., metal bound to organic matter, oxides, or carbonates) (Bendicho et al., 2012a).

For the determination of total elements $(\mathrm{Cd}, \mathrm{Pb}, \mathrm{Cr}, \mathrm{Zn}, \mathrm{Cu}, \mathrm{Hg}$, Se, and rare earth elements), diluted acids are usually employed in combination with sonication by means of ultrasonic baths, probes and cup-horn sonoreactors. While the ultrasonic bath allows the use of a variety of vial sizes (e.g., floating supports for Eppendorf vials), there are also ultrasonic probe devices with different tip diameters depending on the volume to be sonicated (i.e., $2 \mathrm{~mm}$ tip diameter for $150 \mu \mathrm{L}-5 \mathrm{~mL}, 3 \mathrm{~mm}$ for $250 \mu \mathrm{L}-10 \mathrm{~mL}, 6 \mathrm{~mm}$ for $10-50 \mathrm{~mL}$ and $13 \mathrm{~mm}$ for $50-150 \mathrm{~mL}$ ). Probe tips with small diameter produce greater intensity of cavitation, but the energy released is restricted to a narrower area. On the contrary, probe tips with large diameters produce lower intensity but over a greater area, thus permitting larger volume to be sonicated. Finally, the cup-horn sonoreactor is provided with a special adaptor that allows multitreatment for up to 6 Eppendorf vials.

Several protocols using relatively high amounts of sample (0.1-5 g) and high volumes of diluted acids in the range of 3-100 mL were employed, leading to a reduction of time via the use of ultrasound energy. A few examples regarding the use of the ultrasonic bath (for 3-30 min) include the extraction of elements from plant samples (Álvarez et al., 2003; Arruda et al., 2003), soils (Väisänen et al., 2002; Lukkari et al., 2004), biological samples (Liva et al., 2000; Santos et al., 2000; Afridi et al., 2007), hair samples (Bermejo-Barrera et al., 2000) and baby food (Kazi et al., 2010). Other 
examples report the use of the ultrasonic probe (3-20 $\mathrm{min}$ ) for the extraction of elements from biological samples (Afridi et al., 2007; Neves et al., 2009), marine tissues (Costas et al., 2010), leaves (Caballo-López \& Luque de Castro, 2007), plants (Filgueiras et al., 2000), sewage sludge (Hristozov et al., 2004), and rocks (Pumure et al., 2009). It should be pointed out that matrices such as soils, sewage sludge and rocks required higher sonication time than biological tissues for complete element extraction due to their complexity.

The most miniaturized UAE protocols involved a sample mass in the range of 1-30 mg and an extractant volume of 1-2 mL. Several examples were detailed with the three ultrasonic devices mentioned above. The extraction with the ultrasonic bath generally takes 10-60 min for aquatic plant, sewage sludge, mussel tissue, river sediment and tea leaves (Amoedo et al., 1999) and seaweed (Domínguez-Gonzalez et al., 2005). Conversely, the ultrasonic probe requires only 2-15 min for element extraction from animal tissue (Capelo et al., 1998, 1999; Amoedo et al., 1999; Lima et al., 2000; Maduro et al., 2006), hair (Batista et al., 2009), seafood samples (Lavilla et al., 2006b, 2008), biopsy tissues (Lavilla et al., 2006a), sediment samples (Lima et al., 2000), and soils (López-García et al., 2005). Last but not least, the extraction by means of the cup-horn sonoreactor takes 1-45 min for biological tissues (De la Calle et al., 2009, 2012, 2013c) and environmental solid samples (De la Calle, 2011a, 2011b, 2013a).

Although UAE is mostly applied in combination with ETAAS (Capelo et al., 1998; De la Calle et al., 2009), several applications were developed for FAAS (Filgueiras et al., 2000), TXRF (De la Calle et al., 2012, 2013a, 2013c), ICP-OES (Väisänen et al., 2002; Balarama-Krishna \& Arunachalam, 2004; Carvalho-Santos et al., 2009) and ICP-MS (Balarama-Krishna \& Arunachalam, 2004; Batista et al., 2009; Costas et al., 2010). It is worth noting that, in the case of ICP-MS, $5 \mathrm{~mL}$ of extractant (BalaramaKrishna \& Arunachalam, 2004; Costas et al., 2010) or a significant dilution with water after carrying out the extraction (Batista et al., 2009) is required.

Speciation analysis of $\mathrm{As}, \mathrm{Hg}$ and Se using suitable extractants are not truly miniaturized. Usually, they involve extraction using a soft medium (water or diluted $\mathrm{H}_{3} \mathrm{PO}_{4}$ ) and then speciation is performed by HPLC-ICP-MS (Hirata \& Toshimitsu, 2005; Sanz et al., 2005a) or HPLC-HG-AFS (Huerga et al., 2005). When using an ultrasonic probe, lower sonication times (1-5 min) (Huerga et al., 2005; Sanz et al., 2005a) than using the ultrasonic bath (15 $\mathrm{min}$ ) (Hirata \& Toshimitsu, 2005) were reported. Balarama-Krishna et al. (2005) developed a procedure for $\mathrm{Hg}$ and methylmercury extraction from animal and plant tissue and coal fly ash using an ultrasonic probe for 3-4 min. The sample mass employed was $0.1-0.2 \mathrm{~g}$ of sample and $5 \mathrm{~mL}$ of extractant $\left(\mathrm{HNO}_{3}\right.$ +thioruea). Sonication should be followed by selective reduction of species to $\mathrm{Hg}(0)$ prior to analysis, since $\mathrm{SnCl}_{2}$ can reduce only $\mathrm{Hg}$ (II) and $\mathrm{NaBH}_{4}$ can reduce both (Hg(II) and methylmercury).

Several protocols have been designed to evaluate the metal content bound to different geochemical phases in soils and sediments, such as single and sequential extraction schemes from the Standards, Measurements and Testing program from 
the European Commission (SM\&T) (Quevauviller et al., 1993) and the Tessier protocol (Tessier et al., 1979). These schemes allow the separation of the total metal content in different fractions according to their potential mobility and bioavailability. The procedures consist of the dissolution of different solid phases in environmental samples (e.g., organic matter, oxides, carbonates) using specific reagents solutions as extractants for each phase (Filgueiras et al., 2002a). Generally, BCR, Tessier and modified procedures employ large amounts of sample $(1 \mathrm{~g})$, large volumes of reagents (50-200 $\mathrm{mL} \times 3$ or 5 repetitions) and long agitation times (1-48 h) (Tessier et al., 1979; Quevauviller et al., 1993).

Two trends were identified in recent publications of fractionation studies: i) acceleration of the extraction by ultrasound energy but using the same or similar volumes recommended by the standardized protocols and ii) acceleration by ultrasound energy and minimization of volume of reagents and sample mass employed. First, the sample mass used was reduced from $1 \mathrm{~g}$ to 0.5 or $0.25 \mathrm{~g}$ and the volume of reagents to $20 \mathrm{~mL}$ each step. Significant shortening of the extraction times was achieved for BCR and Tessier protocols from several hours to 9-15 min (each step) using an ultrasonic probe (Pérez-Cid et al., 1998) for soils (Pérez et al., 2008), compost (Greenway \& Song, 2002), mud and soils (Marín, 2001), and sediments (Davidson \& Delevoye, 2001), or to 15-30 min using the ultrasonic bath from sewage sludge (Kazi et al., 2006), contaminated soils (Väisänen \& Kiljunen, 2005), or sediments (Davidson \& Delevoye, 2001; Arain et al., 2008). In the case of acetic acid and EDTA extraction, a sample mass of $0.5-3 \mathrm{~g}$ and a reagent volume of up to $75 \mathrm{~mL}$ were used. Moreover, the use of the ultrasonic bath allows one to reduce the operation time from $16 \mathrm{~h}$ to $40 \mathrm{~min}$ for the acetic acid extraction (Krasnodebska-Ostrega et al., 2006), while the use of the ultrasonic probe enabled a processing time reduction from $1 \mathrm{~h}$ to $6-12 \mathrm{~min}$ for the EDTA extraction (Hwang et al., 2007; Remeteiova et al., 2008).

Secondly, the small-scale and accelerated UAE was also developed for single extraction using the ultrasonic probe (Filgueiras et al., 2002b; De la Calle et al., 2013a) and the cup-horn sonoreactor (De la Calle et al., 2013a). The sample mass used was reduced to $25 \mathrm{mg}$ in the BCR protocol, to $0.1 \mathrm{~g}$ in the EDTA extraction and to $25 \mathrm{mg}$ in the acetic acid extraction, with an extraction volume of $1 \mathrm{~mL}$. Using the ultrasonic probe, the time of the procedures was reduced to $0.5-1$ min (each step) in the BCR protocol, to $2 \mathrm{~min}$ for EDTA and $6 \mathrm{~min}$ for the acetic acid extraction. On the contrary, when the cup-horn sonoreactor was used for EDTA and acetic acid extractions, higher sonication time (10 and $30 \mathrm{~min}$, respectively) was required due to the lower intensity of the sonoreactor. Nevertheless, the cup-horn sonoreactor allows for the extraction of up to six Eppendorf vials at a time.

\subsection{Microwave-assisted Extraction}

Microwave energy was first applied in sample preparation for the mineralization or digestion of samples. The matrix is not completely decomposed in MAE, since lower time and softer conditions than in MAD are selected. Microwaves accelerate and enhance 
the extraction by simultanously heating the mixture of extractant (usually an organic solvent) and sample. This is done very fast and without heating the vessel. Choosing a high temperature and an appropriate organic solvent favors the extraction of the analyte in a very short time (Camel, 2000). In fact, MAE provides several advantages over classical techniques, such as i) lower solvent consumption, ii) lower sample usage, iii) reduction in analysis time iv) reduction of contamination, v) analyte loss prevention, vi) more controlled and reproducible results and vii) enhanced operator safety (Camel, 2000). MAE is generally used for organometallic compound extraction from environmental solid samples (Camel, 2000), but several applications for total trace metal analysis, speciation and fractionation in a variety of solid samples have been reported.

MAE has been used for total or pseudo-total metal analysis for diverse environmental samples (including rocks, atmospheric particulate matter, soils, sediments, spruce needles and apple leaves) using nonionic surfactants (Akinlua et al., 2013), mixture of acids (Nieuwenhuize et al., 1991; Zhou et al., 1996a; Borkowska-Burnecka, 2000; Lesniewicz \& Zyrnicki, 2003; Landajo et al., 2004; Kulkarni et al., 2007; Bettiol et al., 2008; Balarama-Krishna et al., 2013), EDTA (Borkowska-Burnecka, 2000; Öztan \& Düring, 2012) and aqua regia (Muránszky et al., 2011; Horváth et al., 2013). Generally, a sample of mass of $0.1-1 \mathrm{~g}$, a volume of $5-40 \mathrm{~mL}$ of acids and an extraction time of 20-45 min were needed for total metal content.

As commented before, MAE has been employed for metal speciation. It should be pointed out that mild extraction conditions should be used to preserve inorganic As species in solution from plankton samples (with water as extractant, for $50 \mathrm{~min}$ ) (Lehman et al., 2013), or diluted $\mathrm{HCl}+\mathrm{H}_{2} \mathrm{O}_{2}$ in food and feed of marine origin (25 min) (Rasmussen et al., 2012). The sample mass ranged from 0.1 to $0.2 \mathrm{~g}$ and the volume of extractants is about $10 \mathrm{~mL}$.

Microwave energy has been applied for the extraction of organometallic compounds from industrial and environmental samples such as textiles, plastics, biological tissues, including such species as organotins (Donard et al. 1995; Szpunar, 1996; Rodríguez et al., 1997; Wang et al., 2006, 2008), methylmercury (Vázquez et al., 1997; Ramalhosa et al., 2001), or arsenic species (Vilanó \& Rubio, 2001) in just 2-10 min. Open vessels are generally preferred for the extraction of organometallic compounds, in order to control the applied microwave energy and prevent the destruction of the carbon-metal bonds (Camel, 2000). Most of the MAE analyses were carried out on a large scale, using sample amounts of $0.15 \mathrm{~g}$ to $5 \mathrm{~g}$ and reagent volumes of $4-40 \mathrm{~mL}$ for organometallics. Usually, low-power microwaves are applied in combination with diverse extractants, e.g., methanol:water (1:1, v/v) (Vilanó \& Rubio, 2001), acetic acid:hexane (1:4, v/v) (Wang et al., 2006), methanol:water (3:2, v/v) (Wang et al., 2008), methanolic potassium hydroxide (Ramalhosa et al., 2001) or methanol (Yang et al., 2010).

Moreover, MAE was used for single extraction tests which involve the use of extractants such as EDTA and acetic acid to obtain the mobilizable and bioavailable fractions from river sediments by FAAS ( $\mathrm{Cu}, \mathrm{Cr}, \mathrm{Ni}, \mathrm{Pb}$, and $\mathrm{Zn}$ ) (Pérez-Cid \& Boia, 2001). Furthermore, MAE was used for metal fractionation. In fact, different sequen- 
tial extraction schemes (SES) such as Tessier and BCR protocols have been enhanced and accelerated by MW energy, giving rise to what have been termed microwave sequential extraction procedures (MSE). MSE generally consists of using the same reagents as in the BCR procedure, but replacing the magnetic agitation by applying MW energy. Several examples can be found in the literature for the extraction of metals from sediments by modified BCR (Ipolyi et al., 2002; Canepari et al., 2005; Arain et al., 2008; Jamali et al., 2009; Alonso-Castillo et al., 2011; Reid, 2011) and Tessier protocols (Perez-Cid et al., 1999; Reid et al., 2011). These approaches were coupled with different analytical techniques such as FAAS (Arain et al., 2008), ETAAS (Arain et al., 2008), ICP-OES (Reid et al., 2011), and ICP-MS (Alonso-Castillo et al., 2011) for the determination of different metals including $\mathrm{Cu}, \mathrm{Ni}, \mathrm{Cr}, \mathrm{Pb}, \mathrm{Zn}$ and $\mathrm{Cd}$. A fractionation method was also performed by continuous-flow sequential extraction assisted by FMW (Nakazato et al., 2006). Most of the BCR and Tessier protocols assisted by microwave energy were carried out on a large scale using sample amounts of $0.25-5 \mathrm{~g}$ and reagent volumes of 8-40 mL. Remarkably, more recent approaches were tested to decrease both sample mass and volume of conventional protocols while maintaining the sample mass-to volume ratio unmodified, following the trend towards miniaturization. For example, the sample mass was reduced from $1 \mathrm{~g}$ to $0.25 \mathrm{~g}$ and the volume of reagents from 40 to $10 \mathrm{~mL}$ in a BCR protocol (Arain et al., 2008; Jamali et al., 2009; Alonso-Castillo et al., 2011) in which the time was reduced from $32 \mathrm{~h}$ to $6 \mathrm{~min}$ (Alonso-Castillo et al., 2011) or to $2 \mathrm{~min}$ (Arain et al., 2008; Jamali et al., 2009) with the aid of MW energy.

\subsection{Accelerated Solvent Extraction}

Accelerated solvent extraction (ASE), also called pressurized solvent extraction (PSE), PLE, high-pressure solvent extraction (HPSE), high-pressure, high temperature solvent extraction (HPHTSE), pressurized hot solvent extraction (PHSE), and subcritical solvent extraction (SSE) involves analyte extraction from solid and semisolid sample matrices under elevated temperature $\left(50-200{ }^{\circ} \mathrm{C}\right)$ and pressure $(500-$ 3000 psi) for short periods of time (5-10 min), using a compressed gas to purge the sample extract to a vessel (Richter et al., 1996; Mesko et al., 2011). Static extractions can be performed at elevated temperatures and pressures or only at elevated pressures in order to avoid decomposition of thermally labile compounds (Szpunar, 2003). Some advantages related to ASE are its high sample throughput, low consumption of solvents, fast extraction cycles, automation with reduced sample handling and suitability for solid and semi-solid samples (Goenaga-Infante et al., 2004).

Although ASE is generally applied for organic compounds, several examples were reported for organometallics and metals (Szpunar, 2003; Mesko et al., 2011), such as As in fish tissue (Mckiernan et al., 1999) and seaweed (Gallagher et al., 2001), Se species in yeast supplements (Goenaga-Infante et al., 2004, 2005) and multielemental analysis of lubricating oil (Carballo-Paradelo et al., 2012). Methanol and water are the usual extractant phases. 
In most of cases, the sample mass used is 0.1-0.7 $\mathrm{g}$ and the volume of extractant added is $10 \mathrm{~mL}$ (McKiernan et al., 1999; Goenaga-Infante et al., 2004, 2005; CarballoParadelo et al., 2012). However, a $3 \mathrm{~mL}$ ASE cell, smaller than the conventional one, allowed the miniaturization of the ASE approach (Gallagher et al., 2001). The solvent involved in the miniaturized ASE approach was $\mathrm{MeOH}: \mathrm{H}_{2} \mathrm{O}(1: 1, \mathrm{v} / \mathrm{v})$ (Gallagher et al., 2001). Usually, 3 or 6 cycles of 5 min extraction time were performed at 1500 psi (McKiernan et al., 1999; Gallagher et al., 2001; Goenaga-Infante et al., 2004, 2005). The temperature was fixed at $100{ }^{\circ} \mathrm{C}$ and diatomaceous earth was added as dispersing agent (Goenaga-Infante et al., 2004, 2005).

Recently, a laboratory-made miniaturized device for PLE was patented for the extraction of As(III), As(V) and total As from hair samples (Sanz et al., 2007). About $50 \mathrm{mg}$ of hair sample was mixed with $350 \mathrm{mg}$ of dispersant medium. The homogenized mixture was then introduced into the extraction cell, which consisted of a stainless steel liquid chromatographic column. The PLE device was equipped with an homemade oven whose temperature was controlled by a digital electronic controller. The extraction cell was mounted between two valves. One valve was connected to the HPLC pump that introduced the extraction solvent in the cell and controlled the pressure in the system, while the second valve was connected to the collection vial (Sanz et al., 2007).

\subsection{Supercritical Fluid Extraction}

Supercritical fluid extraction (SFE) consists of the extraction of analytes from a matrix using a supercritical fluid as the extraction solvent. While in off-line SFE hundreds of $\mathrm{mg}$ to $\mathrm{g}$ of sample are used, in on-line SFE only a few $\mathrm{mg}$ of sample are extracted. A complexing agent is required in order to form neutral metal complexes and facilitate the extraction, usually dithiocarbamate. In general, $\mathrm{CO}_{2}$ is utilized as supercritical fluid extractant because it is chemically inert, non-toxic, non flammable and noncorrosive. Moreover, a modifier could be used, for example 1-10 \% (v/v) MeOH, acetic acid or formic acid.

Apart from avoiding the use of organic solvents, SFE avoids or reduces the formation of wastes. However, SFE is not widely applied and the energy cost needed to maintain a substance in the supercritical state is very high (Bendicho et al., 2011).

Even though SFE is more commonly used for organic compounds (e.g., polycyclic aromatic hydrocarbons (PAHs) and polychlorinated biphenyls), different elements have been extracted from wood ( $\mathrm{Cr}, \mathrm{Cu}, \mathrm{As})$, fly ash ( $\mathrm{Zn}, \mathrm{Pb}, \mathrm{Mn}, \mathrm{Cd}, \mathrm{Cu}, \mathrm{V}, \mathrm{Sb}, \mathrm{Ni}$, Mo, Cr and Co) (Lin et al., 2014) and sand (S. Wang et al., 2003, Yabalak \& Gizir, 2013) by SFE using diverse chelating agents at 100-200 bar and $40-130{ }^{\circ} \mathrm{C}$. Furthermore, the SFE of organometallic species of $\mathrm{Sn}, \mathrm{Pb}, \mathrm{Hg}$ and As after derivatization was also reported in the literature (Alzaga et al., 2003). The application of supercritical $\mathrm{CO}_{2}$ in heavy metal extraction has been recently reviewed (Sunarson \& Ismadji, 2009; Umale \& Mahanwar, 2010; Lin et al., 2014). 
As a novel approach, SFE was adapted for improving fractionation studies of a three-step BCR sequential extraction procedure (SEP) of lake and river sediments (Horváth et al., 2013). The tested procedure consisted of a three-step method for the extraction of different fractions. Thus, $\mathrm{CO}_{2}$-soluble organic fraction and sulfides were first extracted by means of supercritical $\mathrm{CO}_{2}$, while water-soluble fraction and a fraction bound to carbonates where subsequently obtained by using subcritical $\mathrm{H}_{2} \mathrm{O}$ and subcritical $\mathrm{H}_{2} \mathrm{O}+\mathrm{CO}_{2}$, respectively. Each step was performed at $80{ }^{\circ} \mathrm{C}$ and $27 \mathrm{MPa}$ for $30 \mathrm{~min}$, but without the addition of organic solvents, thus involving a solventless procedure. The sample mass used was $0.5 \mathrm{~g}$.

\subsection{Matrix Solid-phase Dispersion}

MSPD consists of the disruption of a sample by mechanical blending using a solid support or a dispersing agent (silica-based support) bonded phase which is suitable for extraction. After blending, the mixture is placed inside a cartridge or column. A new sample matrix-solid support phase is formed and analytes tend to be more weakly bonded to it. Then, the analytes are eluted using different solvents by gravity or by applying pressure or vacuum (Kristenson et al., 2006). This approach was developed as an alternative to conventional techniques such as Soxhlet extraction or LLE that require higher amounts of sample, volume of eluents and time (Moliner-Martinez et al., 2009). Moreover, MSPD avoids the problem of emulsion formation in LLE (Ramos, 2012). MSPD is, in general, less time consuming than Soxhlet extraction, and shows higher extraction efficiencies (EEs) than achieved by conventional LLE (Capriotti et al., 2013).

Several reviews are focused on the MSPD technique (Barker, 2000; Ramos et al., 2005; Kristenson et al., 2006; Barker, 2007; Moreda-Piñeiro et al., 2009; Ramos, 2012; Caprioti et al., 2010, 2013). A scheme of MSPD is shown in Figure 7.6.

Several parameters should be taken into account when optimizing a MSPD method, including the nature of the solid support, the bonded phase, the components of the matrix, and the polarity of the eluting solvents (Moreda-Piñeiro et al., 2009). Several sorbents have been described in the literature, including molecularly imprinted polymers (MIPs) and carbon nanotubes (CNTs) (Caprioti et al., 2013). Other options that increase the analyte recovery are the use of ultrasound energy for improving the EE (Capriotti et al., 2010) and the use of high-temperature and high-pressure solvents that facilitate the automation of MSPD (Kristenson et al., 2006). Moreover, interfering components of the matrix could be selectively retained on the column by interactions with the stationary phase (Ramos, 2012).

Although MSPD has been designed for organic compound purification and extraction (Ramos et al., 2005, 2009; Ramos, 2012), it is also a promising and appealing sample pre-treatment technique for trace elemental analysis. Several applications have been reported in the literature for inorganic and organometallic compounds (Moreda-Piñeiro et al., 2008, 2009; Martínez-Fernández et al., 2011), and they will be described in detail below. 


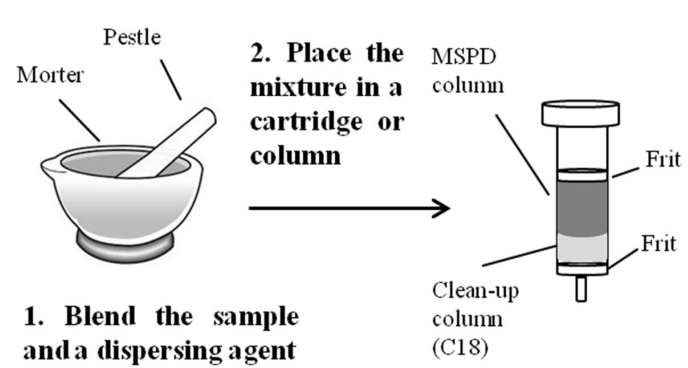
and a dispersing agent

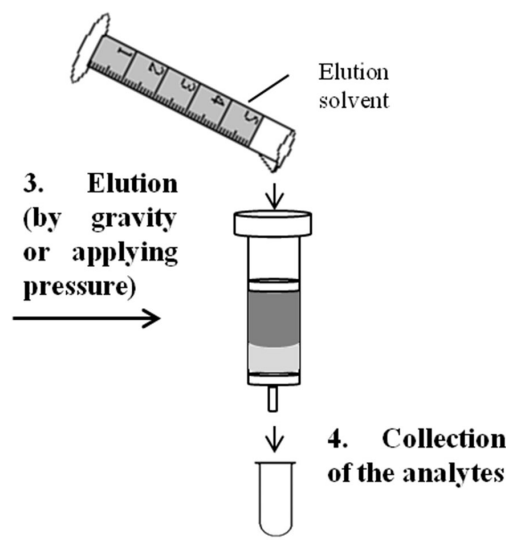

Figure 7.6 Scheme of MSPD including the different steps.

MSPD is considered advantageous from different points of view. Firstly, MSPD requires a small amount of sample (0.1-0.25 g) versus conventional techniques (10 g) (Moliner-Martínez et al., 2009), an important consideration when only small amount of sample is available (Kristenson et al., 2006). Secondly, a relatively small volume of solvent (2 mL) is needed (Moliner-Martínez et al., 2009), which is generally lower than reported in classical methods (hundreds of $\mathrm{mL}$ ) (Ramos et al., 2005; MolinerMartínez et al., 2009). In fact, it has been reported that MSPD requires 95\% less solvent compared to classical approaches (Moreda-Piñeiro et al., 2009). Additionally, MSPD is shorter (less than $1 \mathrm{~h}$ ) than classical methods (several hours) (Moliner-Martinez et al., 2009), with some researchers reporting that MSPD requires $90 \%$ less time than classical methods (Moreda-Piñeiro et al., 2009). Moreover, MSPD offers the possibility of performing several processes in one step, involving extraction and cleanup, resulting in a faster approach (Kristenson et al., 2006). MSPD is also a low cost, simple technique that does not require any special instrumentation (Ramos et al., 2005).

The applications dealing with MSPD for the determination of metals and organometallics (Moreda-Piñeiro et al., 2009; Capriotti et al., 2013) include Cd, Co, Cr, Mn, $\mathrm{Ni}, \mathrm{Sr}$ and $\mathrm{Zn}$ in lake and estuarine sediments (Martínez-Fernández et al., 2011), $\mathrm{Sb}(\mathrm{III})$ and $\mathrm{Sb}(\mathrm{V})$ in marine sediment (Moreda-Piñeiro et al., 2009), As(III) and As(V) in seafood (Moreda-Piñeiro et al., 2009, 2012) and organometallics such as arsenobetaine (AsB), arsenocholine (AsC), monomethylarsonic acid (MMA), and dimethylarsinic acid (DMA) in seafood samples (Moreda-Piñeiro et al., 2009, 2012). For the MSPD extraction of inorganic and organic species of As in seafood samples (MoredaPiñeiro et al., 2009, 2012), $0.25 \mathrm{~g}$ of sample was blended with diatomaceous earth $(1.75 \mathrm{~g})$ and the mixture packed into a cartridge containing $2 \mathrm{~g}$ of $\mathrm{C}_{18}$ as a co-sorbent. After that, arsenic species were eluted with $10 \mathrm{~mL}$ of methanol:water (1:1, v/v), con- 
centrated by rotary evaporation to approx $2 \mathrm{~mL}$ and determined by anion-exchange HPLC-ICP-MS.

As discussed in previous sections, SES or SEP have been accelerated by ultrasound and microwave energy (Sutherland, 2010). MSPD has also been applied for evaluating the partitioning of metals ( $\mathrm{Cd}, \mathrm{Co}, \mathrm{Cr}, \mathrm{Mn}, \mathrm{Ni}, \mathrm{Sr}$ and $\mathrm{Zn}$ ) in lake and estuarine sediments prior to ICP-MS analysis (Martínez-Fernández et al., 2011). The SES approach begins by mixing $0.5 \mathrm{~g}$ of sample with $1.0 \mathrm{~g}$ of a dispersing agent (such as alumina, silicates, or diatomaceous earth) and blending the mixture with a mortar and pestle for $5 \mathrm{~min}$. Then, the dispersed sample is packaged inside a disposable syringe and placed in a vacuum manifold station. The extractants conventionally used in the BCR protocol (0.11 mol L $\mathrm{m}^{-1}$ acetic acid, $0.1 \mathrm{~mol} \mathrm{~L}^{-1}$ hydroxylammonium chloride and $8.8 \mathrm{~mol}$ $\mathrm{L}^{-1}$ hydrogen peroxide $+1 \mathrm{~mol} \mathrm{~L}^{-1}$ ammonium acetate) are then passed through the sample. The time of the procedure and the volume of reagents were reduced in relation with the conventional approach (Sutherland, 2010) from $51 \mathrm{~h}$ to $3-4 \mathrm{~h}$ the whole procedure and from $40 \mathrm{~mL}$ to $10 \mathrm{~mL}$ and $25 \mathrm{~mL}$ in the first and second steps, respectively. The main disadvantage of this procedure (Martínez-Fernández et al., 2011) is the washing (or rinsing) step required after each extraction which involves passing $10 \mathrm{~mL}$ of water through the column and takes $20 \mathrm{~min}$. Moreover, inaccuracy was observed for the reducible fraction, as occurs in the conventional, ultrasound and microwave-assisted BCR protocols.

\subsection{Solid-phase Microextraction}

SPME is a miniaturized sample preparation technique first introduced by Pawliszyn and co-workers in the early 1990s. Generally, SPME is mainly used to extract organic compounds from solutions as well as headspace. In the case of headspace enrichment, samples can be of solid or liquid nature. SPME is a solventless sample preparation technique, since no addition of solvent is required in headspace solid-phase microextraction (HS-SPME). The procedure involves two integrated steps: i) mass tranfer of the analytes from the solid sample to the headspace by heating and ii) preconcentration of the analytes in the SPME fiber by exposing it to the headspace. After that, sample introduction is generally performed by thermal desorption of analytes into an analytical instrument such as a GC. A detailed description of SPME techniques can be found in chapter 2 of this book.

HS-SPME has been applied to solid samples, such as marine sediment, dogfish muscle and liver tissue and the volatile metals and organometallics can be collected onto the fiber (Figure 7.5E). It is necessary to heat the solid sample to promote the volatilization of elements of interest. This approach was tested for the analysis of As (as AsB), Se, $\mathrm{Hg}$ (as methylmercury), $\mathrm{Pb}$ (as trimethyllead) and $\mathrm{Sn}$ (as butyltin species, mono-, di- and tributyl tin) by ICP-MS. $1 \mathrm{~g}$ of solid sample was placed in a vial and heated on a hot plate to a temperature between $70-150{ }^{\circ} \mathrm{C}$ for a period of $1-5 \mathrm{~min}$ (Mester, 2002). A similar approach was employed for the analysis of organotin compounds by SPME-ICP-TOF-MS from sediment and urban dust by simply increasing the 
temperature to about $40{ }^{\circ} \mathrm{C}$ (Mester \& Sturgeon, 2002). This approach is completely green, since no chemicals were used.

\subsubsection{Combined Techniques}

A wide range of miniaturized sample preparation approaches that are generally applied to liquid samples cannot be directly used for the analysis of solid and semisolid samples. Thus, solid samples have to be converted into liquid samples by diverse procedures such as digestion, extraction or ashing prior to another sample preparation procedure such as SPE, SPME, LPME, or prior new generation miniaturized analytical systems including $\mu$-TAS or LOC.

\subsection{Solid-phase Extraction}

SPE is frequently used for the preconcentration or clean-up of target analytes from aqueous samples. SPE is an alternative sample preparation technique to LLE which can reduce the volume of solvents needed. SPE involves the extraction of target compounds from an aqueous sample, extract or a digested solid sample (Camel, 2003). Conventional SPE uses solid phases such as $\mathrm{C}_{18}$-bonded silica and the required volume of solvents ranges from $1 \mathrm{~mL}$ to $1 \mathrm{~L}$ depending on the scale of the system.

Generally, solid samples are first digested or dissolved and then subjected to SPE using cartridges, syringe barrels, microcolumns or disks (Camel, 2003). Several examples in the literature involve the analysis of geological materials ( $\mathrm{Pu}$ et al., 1998) and biological tissues (Yin et al., 1998).

CNTs that were previously oxidized were used as sorbents in SPE for the analysis of metals and organometallics in solid samples (Muñoz et al., 2005b; Ravelo-Pérez et al., 2010; Herrero-Latorre et al., 2012). Some of the samples (i.e., cigarettes, biological samples, garlic, canned fish, leaves, human hair, soils) were digested by MAD (Teixeira-Tarley et al., 2006; Barbosa et al., 2007; Tuzen et al., 2008), while soils, human hair, tea and rice were extracted by sonication $\left(\mathrm{H}_{2} \mathrm{SO}_{4}: \mathrm{HNO}_{3}(3: 1, \mathrm{v} / \mathrm{v})\right.$ for $2 \mathrm{~h}$ and stirred at $55^{\circ} \mathrm{C}$ for $7 \mathrm{~h}$ prior to SPE (Liu, 2008). The amount of CNTs in the different procedures was in the range of $10-300 \mathrm{mg}$, and the volume of sample after treatment was in the range of 25-400 mL. The enrichment factors (EFs) achieved varied from 30 to 200. Usually, the elution of analytes is carried out by adding diluted $\mathrm{HNO}_{3}$. The analytical technique most commonly employed was FAAS (Teixeira-Tarley et al., 2006; Barbosa et al., 2007; Liu et al., 2008, 2009; Chen et al., 2009), although a few applications using ICP-OES (Zang et al., 2009) and ICP-MS (Chen et al., 2009) were also published.

Another related technique is dispersive micro solid-phase extraction (DMSPE). The use of multiwalled carbon nanotubes (MWCNTs) as a solid sorbent for the DMSPE preconcentration of Se from biological tissues was recently reported (Skorek et al., 2012). First, $250 \mathrm{mg}$ of solid sample was digested in $5 \mathrm{~mL}$ of $\mathrm{HNO}_{3}$ by $\mathrm{MAD}$. $\mathrm{HCl}$ was added to reduce Se(VI) to Se(IV) and DMSPE was performed prior to XRF analysis. For 
DMSPE, $100 \mathrm{~mL}$ of the digested sample was mixed with $100 \mu \mathrm{L}$ of a solution of APDC and $200 \mu \mathrm{L}$ of $5 \mathrm{mg} \mathrm{L}^{-1}$ dispersed oxidized MWCNTs, and stirred for $5 \mathrm{~min}$ at $700 \mathrm{rpm}$. After that, the sample was filtered and MWCNTs containing the adsorbed metal chelates that remained in the filter were dried under IR radiation and directly analyzed.

As a modification of SPE, another interesting improvement is the use of SS for preconcentration analysis using solid phases as sorbents. However, solid samples had to be previously digested by MAD. Several recent studies reported the use of activated carbon as a sorbent for the extraction and preconcentration of $\mathrm{Au}$ from geological samples (Dobrowolski et al., 2012), Ni and Co from plant materials (Dobrowolski \& Otto, 2012), and Se in complementary feed (Dobrowolski \& Otto, 2013). Separation and enrichment of metals consisted of the adsorption of metal ions onto activated carbon from aqueous solutions. After adsorption, SS introduction replaced the step of elution of analytes, thus avoiding sample dilution and, as a consequence, decreased EFs. Powdered activated carbon was chemically modified, followed by drying and washing steps. Different protocols can be employed for the modification of activated carbon by: i) treatment with $\mathrm{H}_{2} \mathrm{O}_{2}$ and $\mathrm{HNO}_{3}$ (Dobrowolski et al., 2012), ii) treatment with $\mathrm{HNO}_{3}$ and outgassing in the argon atmosphere at high temperature (1100 $\left.{ }^{\circ} \mathrm{C}\right)$ followed by impregnation with dimethylglyoxime solution (Dobrowolski \& Otto, 2012), and iii) loading with Fe species by impregnation method and thermo-chemical reactions using different concentrations of $\mathrm{Fe}\left(\mathrm{NO}_{3}\right)_{3}$ (Dobrowolski \& Otto, 2013). Thus, modified activated carbon was used for adsorption of the target elements. Firstly, previously digested samples were evaporated almost to dryness. Then, 0.1-0.2 g of the modified activated carbon was mixed with the sample. The suspension was filtered and dried at $120^{\circ} \mathrm{C}$. Finally, after preconcentration of metals onto modified carbon, the determination of metals is performed in a suspension of carbon. Thus, the carbon suspension was prepared in a range of $1-50 \mathrm{mg} \mathrm{mL}^{-1}$ in $5 \% \mathrm{v} / \mathrm{v} \mathrm{HNO}_{3}$ as a liquid medium, homogenized by Vortex agitator and introduced in a graphite furnace by GFAAS (Dobrowolski et al., 2012; Dobrowolski \& Otto, 2012, 2013).

\subsection{Headspace Solid-phase Microextraction}

Different protocols have been designed for the enrichment of metals and organometallic compounds by HS-SPME. Although the extraction of elements from the sample to the aqueous phase is not miniaturized, the preconcentration process involves a miniaturized procedure. Derivatization reactions are typically used to obtain volatiles to be extracted by the SPME fiber when dealing with the enrichment of metals and organometallic compounds. For example, SPME has been used for the determination of methylmercury in biological samples. Tissue samples $(0.25 \mathrm{~g})$ were extracted using $5 \mathrm{~mL}$ of $3 \mathrm{~mol} \mathrm{~L}^{-1} \mathrm{HCl}$ and diluted to $30 \mathrm{~mL}$. Then, the SPME fiber was exposed to the headspace of these extracts without the need of centrifugation step to capture methylmercury for subsequent ICP-MS analysis (Mester et al., 2000). In another study, a digestion step was incorporated prior to HS-SPME for Hg determination in biological tissues using a Pd wire instead of a common fiber (Romero et al., 2010). $200 \mathrm{mg}$ of 
sample were digested using $2 \mathrm{~mL} \mathrm{HNO}_{3}+1 \mathrm{~mL} \mathrm{H}_{2} \mathrm{O}_{2}$ by MAD. Then, the Pd wire was placed in the headspace for $30 \mathrm{~min}$ and analyzed by CVAAS. Another study analyzed butyltin in sediment samples by SPME combined with GC-atomic emission detection (Carpinteiro et al., 2004). 0.1-0.5 g of sample was sonicated with $5 \mathrm{~mL}$ of acetic acid and neutralized with ammonium hydroxide. After derivatization, SPME was carried out in the headspace mode for $10 \mathrm{~min}$ at room temperature.

Determination of methylmercury and mercury (II) in sediment and biota (fish, crab, prawn and bivalves) samples was also performed by SPME-GC-MS (Mishra et al., 2005). Here, $0.5 \mathrm{~g}$ of sediment sample was extracted using $15 \mathrm{~mL}\left(\mathrm{HCl}: \mathrm{CH}_{3} \mathrm{OH}\right.$, 1:1 (v/v) and sonicated for $2 \mathrm{~h}$. Similarly, for biota samples, $0.3-0.8 \mathrm{~g}$ of edible tissue were first hydrolyzed using $25 \%(\mathrm{~m} / \mathrm{v}) \mathrm{KOH}(10-20 \mathrm{~mL})$ for $2 \mathrm{~h}$ under ultrasonic treatment. After the extraction, the preconcentration of analytes was performed by exposing the SPME fiber to the headspace for $15 \mathrm{~min}$ at $40{ }^{\circ} \mathrm{C}$ after alkylation of mercury species. Another example of mercury speciation analysis involving SPME was based on the combination of HS-SPME with furnace atomization plasma emission spectrometry in biological samples (Gringberg et al., 2003). A 0.25 g sample of biological tissue was mixed with $25 \mathrm{~mL}$ of $25 \%$ (m/v) methanolic $\mathrm{KOH}$ solution for $4 \mathrm{~h}$. For HS-SPME sampling, $12.5 \mathrm{~mL}$ of acetate buffer $(\mathrm{pH}=5)$ was added to 200$1000 \mu \mathrm{L}$ of the dissolved sample along with $1 \mathrm{~mL}$ of derivatizing agent. The mixture was magnetically stirred to ensure the proper mixing of the sample solution and to enhance transfer of the analytes from the solution to the headspace. After a predetermined time (20-200 s), the SPME fiber was introduced into the vial headspace (at a temperature in the range of $22-55^{\circ} \mathrm{C}$ depending on the mercury species) for enrichment. Organomercury and organotin compounds were also determined in sediment and biota samples by GC with microwave-induced plasma atomic emission detection using HS-SPME for preconcentration (Delgado et al., 2008). In this case, the extraction of these compounds from the sample involved the use of $0.2-1 \mathrm{~g}$ of sediment extracted under sonication in $10 \mathrm{~mL}$ of diluted $\mathrm{HCl}$ in an ultrasonic bath for $1 \mathrm{~h}$. Conversely, the extraction from the biological tissue used a sample mass of 0.1-0.3 $\mathrm{g}$ and extraction in an ultrasonic bath for $1 \mathrm{~h}$ with a diluted $\mathrm{KOH}$ in $\mathrm{MeOH}$ solution. SPME extraction involved a 3-24 min headspace exposure time, temperatures between $20-90{ }^{\circ} \mathrm{C}, 1-10 \mathrm{~min}$ desorption time, $150-260^{\circ} \mathrm{C}$ desorption temperature and sample volumes between $5-22 \mathrm{~mL}$. A fiber with a $30 \mu \mathrm{m}$ coating of polydimethylsiloxane (PDMS) was used in this case.

\subsection{Liquid-phase Microextraction}

LPME, also named solvent microextraction (SME), involves the miniaturization of conventional LLE (Pena-Pereira et al., 2009, 2010a, 2010b). LPME is most frequently uses to analyzed aqueous solutions due to their relative simplicity and low matrix effects. More efforts are needed to extend the applicability of LPME to solid matrices. Digestion of the solid or extraction to an aqueous phase is usually performed prior to LPME. A detailed description of LPME techniques can be found in chapter 4 of this 
book. In this section, the treatment step prior to different modes of LPME of solid samples will be discussed.

One mode of LPME, headspace single-drop microextraction (HS-SDME), was applied to extract methylmercury from fish tissue by in situ hydride generation and ETAAS (Gil et al., 2005). First, methylmercury was selectively extracted from fish tissue samples $(0.4 \mathrm{~g})$ by means of an ultrasonic probe for 2-5 min using $2 \mathrm{~mol} \mathrm{~L}^{-1}$ $\mathrm{HCl}$. Generation of methylmercury hydride was performed in a closed vial containing $5 \mathrm{~mL}$ of $0.1 \mathrm{~mol} \mathrm{~L}^{-1} \mathrm{NaOAc} / \mathrm{HOAc}$ buffer. An aqueous drop of $3 \mu \mathrm{L}$ containing Pd(II) or $\mathrm{Pt}(\mathrm{IV})$ was selected as trapping agent. After $3 \mathrm{~min}$ of extraction and with agitation of the sample, methylmercury was preconcentrated in the drop. A similar approach was reported for the GC analysis of organotin species from extracts of sediment samples using an organic drop containing $2 \mu \mathrm{L}$ of decane (Colombini et al., 2004).

Dispersive liquid-liquid microextraction (DLLME) was also applied to solid samples. A pre-treatment step, ranging from conventional AD to small-scale UAE, was required. In the first case, acid digestion of soil samples $(1 \mathrm{~g})$ was carried out with $2 \times 10 \mathrm{~mL}$ of aqua regia for $1 \mathrm{~h}$ followed by the addition of $1 \mathrm{~mL}$ of $\mathrm{HF}+1 \mathrm{~mL}$ of $\mathrm{HCl}$, and diluted to $25 \mathrm{~mL}$, whereas only $1 \mathrm{~mL}$ was used for the preconcentration of Pd by DLLME (Shamsipur et al., 2009). In addition, a miniaturized procedure consisting of the combination of UAE and DLLME for determination of Au in soils and sediments by ETAAS was recently reported (De la Calle et al., 2011b). Specifically, a portion of solid sample of 3-30 mg was weighed into an Eppendorf vial and $1 \mathrm{~mL}$ of a $25 \%$ (v/v) $\mathrm{HNO}_{3}$ $+25 \%(\mathrm{v} / \mathrm{v}) \mathrm{HCl}$ mixture was added to extract Au. Ultrasound energy was applied for $20 \mathrm{~min}$ by means of a cup-horn sonoreactor. After sonication, centrifugation was carried out for $2 \mathrm{~min}$ at $5000 \mathrm{rpm}$. The preconcentration step was then performed using $900 \mu \mathrm{L}$ of the extract.

Another miniaturized sample preparation technique generally applied for water samples is ultrasound-assisted emulsification microextraction (USAEME). USAEME has been used to extract previously digested solid samples such as plant tissues followed by ICP-OES determination of Al, Cu, Fe and $\mathrm{Zn}$ (Sereshti et al., 2011). In addition, soil and sediment samples can be directly extracted using ultrasound energy and used to quantify Au by ETAAS (De la Calle et al., 2011b). Hollow-fiber liquid-phase microextraction (HF-LPME) was also applied for the preconcentration of trace elements ( $\mathrm{Cu}, \mathrm{Zn}, \mathrm{Pd}, \mathrm{Cd}, \mathrm{Hg}, \mathrm{Pb}, \mathrm{Bi}$ ) from environmental and biological samples (peach leaves) after pre-treatment of the samples coupled to ETV-ICP-MS (Xia et al., 2007).

\subsection{Fully Miniaturized Analytical Systems}

Several reviews (Miró \& Hansen, 2007; Mark et al., 2010) and a book (Ríos et al., 2009) are focused on fully miniaturized analytical systems. All of these advanced miniaturized devices share common characteristics such as the very low volume of reagents $(\mu \mathrm{L}, \mathrm{nL}, \mathrm{pL})$ used. In addition to miniaturization, integration and automation are other key working principles. This section includes applications of $\mu$-TAS, LOC, LOV and lab-on-disk (LOD) devices. It should be highlighted that fully miniaturized ana- 
lytical systems are generally applied for aqueous samples and, in the case of solid samples, an off-line sample preparation is required.

$\mu$-TAS, also named LOC, have been applied to trace metals analysis from aqueous samples such as river and drinking water samples (Xue et al., 2013; Ibarlucea et al., 2013) and fruit juices (Chailapakul et al., 2008). LOC was also combined with ICP-MS (Al-Suhaimi, 2011). In this case, a downscaled SPE device was coupled to ICP-MS using a PTFE connector and coupled with a flow injection manifold for determination of Cd, Co and Ni in seawater samples. Some examples found in the literature for LOC analysis of solid samples (e.g., environmental and food samples), are summarized here. However, off-chip sample preparation is usually performed due to the difficulty to directly incorporate a complex matrix into a microchip (Ríos et al., 2009). More research is highly required in relation with the on-chip treatment of solid samples.

Chang et al. (2005) designed a miniaturized sensor for monitoring $\mathrm{Pb}$ (II) in environmental samples. In this study, electroplating sludge samples were analyzed according to US EPA Method 3050B (AD of sediments, sludges and soils. Method 3050B, 1996), involving off-chip extraction, centrifugation and filtration. Then, the sample was diluted in a solution of ammonium hydroxide before injection on the microchip. This microdevice (50 $\mu \mathrm{m}$ width, $30 \mu \mathrm{m}$ depth and $14 \mathrm{~mm}$ length) made of PDMS, contained a network of microfluidic channels that were fluidically coupled via a nanocapillary array interconnect. The selectivity of the sensor for $\mathrm{Pb}$ (II) was obtained using a lead-specific DNAzyme followed by laser-induced fluorescence as the detection mode. The limit of detection achieved for $\mathrm{Pb}$ (II) was $11 \mathrm{nM}$. It should be pointed out that despite the very small volume $(<100 \mathrm{pL})$ still allowed efficent molecular recognition reactions to occur.

A capillary electrophoresis (CE) microchip was fabricated for the separation and detection of $\mathrm{Cd}, \mathrm{Pb}, \mathrm{Cu}, \mathrm{Co}, \mathrm{Ni}$ and $\mathrm{Hg}$ (Deng \& Collins, 2003). Firstly, a spiked sample was deposited onto a Plexiglas surface. After evaporation, the surface was washed with $0.1 \mathrm{~mol} \mathrm{~L}^{-1} \mathrm{HNO}_{3}$ and different reagents including an special chelating agent were added for the formation of metal chelates. The resulting sample can be directly introduced into the CE microchip, or preconcentrated by off-line SPE (using a C18 silica gel microcolumn) and subsequently separated by CE microchip. The combination of surface sampling and SPE coupling with CE allows the improvement of detection limits by 100 -fold.

He et al. (2007) developed a method to determine nitrite in different food samples (e.g., vegetables, fruits and meats) using a poly(methylmethacrylate) $(50 \times 40 \times 5 \mathrm{~mm})$ substrate. For the treatment of solid samples, off-chip extraction with a 732-Cation Resin column and filtration was employed. A microflow injection analysis ( $\mu$-FIA) system was used on a chip with chemiluminescence detection using the luminol-ferricyanide system. The volume of reaction area was about $1.8 \mu \mathrm{L}$.

An electrochemical sensor with a microfabricated on-chip Bi electrode was designed for in situ heavy metal ion ( $\mathrm{Cd}$ and $\mathrm{Pb}$ ) determination in soil pore, ground water and cell culture media (Zou et al., 2008). In this work, soil pore and ground 
water samples were taken from a lab-scale reactor for in situ measurements to simulate the pollution of the soil pore and ground water of the heavy metal wastes disposable sites. Thus, a solution of Cd(II) was flowed through the soil and the groundwater was collected. The system consisted of a microfluidic chip with microchannels, syringe pumps (for sample loading), Bi working electrode, $\mathrm{Ag} / \mathrm{AgCl}$ reference electrode and gold counter electrode (each electrode was $3 \mathrm{~mm}$ in length). Moreover, this sensor is disposable and easy to use, has very low cost, is able to be mass produced, involves very small analyte consumption and generates a very small amount of waste.

Another procedure was reported for the determination of nitrite in food samples, namely sausages and ham (Shiddikiy et al., 2009). In this case, off-line sample preparation encompassed two possibilities - sonication (i.e., $2.5 \mathrm{~g}$ of sample in $50 \mathrm{~mL}$ extractant, sonication time of 5-10 $\mathrm{min}$ ) and MAE (1 g of sample in $2 \mathrm{~mL}$ extractant, heated in a microwave oven for 1-3 $\mathrm{min}$ ). The extracted samples were analyzed using microchip electrophoresis with electrochemical detection (MCE-ED) using a modified carbon paste electrode. The time required for analysis was less than $200 \mathrm{~s}$.

Recently, a chip-based LPME was fabricated and combined with ETV-ICP-MS for the determination of trace metals ( $\mathrm{Cu} . \mathrm{Zn}, \mathrm{Cd}, \mathrm{Hg}, \mathrm{Pb}$ and $\mathrm{Bi}$ ) in human hair and cell samples (Wang et al., 2013a). DDTC was used both as the chemical modifier for low-temperature ETV-ICP-MS and as a chelating reagent using octanol as the organic phase for the chip-based LPME. Double "Y" type microchannels were designed. The chip had two inlet branch channels (one for aqueous phase and another for organic phase), one central channel for LPME (where the aqueous and organic phase combine in a laminar flow) and two outlet branch channels (one for aqueous phase and another for organic phase). Syringe pumps were applied for the introduction of two phases. These two phases formed laminar flow in the central channel, increasing the interfacial contact between the phases, favoring the entrance of metal chelates into the organic phase (Figure 7.5F). The extraction took $3.5 \mathrm{~min}$ and $7 \mu \mathrm{L}$ of the organic phase was collected and subsequently introduced into the graphite furnace for ETVICP-MS analysis. Limits of detection (LODs) were in the range of 6.6-89.3 $\mathrm{pg} \mathrm{mL}^{-1}$ while sample recoveries in the range of $87-119 \%$ were achieved. The developed method was fast, suitable for microsamples and exhibited low reagent consumption.

LOV constitutes the third generation of flow injection techniques, showing the significant progress that has been made in miniaturization, automation and integration of on-line sample pre-treatment (Yu et al., 2011). Several reviews related to LOV can be found in the literature (J. Wang et al., 2003; Chen \& Wang, 2007; Yu et al., 2011).

LOV was combined with instruments like ETAAS (Wang \& Hansen, 2001a; Long et al., 2005) and ICP-MS (Wang \& Hansen, 2001b) for total metal detection (Ni, Bi) and metal speciation ( $\mathrm{Cr}(\mathrm{III}), \mathrm{Cr}(\mathrm{VI})$ ) in order to automate the process and downscale sample and reagent consumption. This procedure can be applied straightforwardly for aqueous samples (Long et al., 2005) but digestion with $\mathrm{HNO}_{3}+\mathrm{HF}$ is required for solid samples, such as ash and river sediment (Wang \& Hansen, 2001a, 2001b). LOV facilitates processes such as mixing, dilution, etc. It is worth noting that volumes 
involved in LOV are in the range of microliter to sub-microliter. Thus, its combination with ETAAS is suitable, but it involves a challenge in the case of ICP-MS, which requires larger volumes (mL) for analysis. Wang and Hansen (2001b) developed a homemade direct injection high efficiency nebulizer to solve this problem which allowed the ICP-MS to detect metals after aspirating only $15 \mu \mathrm{L}$ of sample.

The lab-on-a-valve bead-injection (LOV-BI) sample pre-treatment emerged to solve several inconveniences of SPE (e.g., long operation time, clogging of the column and deactivation of the SPE material) and to facilitate the separation and preconcentration of metal ions using different sorbent materials. In the BI technique a packed microcolumn is generated in situ by aspirating into the system a suspended solution of microcarrier beads from a peripherical port of the valve. Two channels act as microcolumns positions connected with a small tubing. This tubing present two functions: a) holding the beads inside the column reactor avoiding escaping of the beads and b) automatically transporting of the beads between different column positions. Advantages of LOV-BI include: i) sorbent materials which are renewed after each cycle, ii) matrix interferences are avoided, iii) flow resistance is eliminated and iv) analytical performance is improved. LOV-BI has been coupled to different atomic spectrometric techniques for metal analysis, as reported in a recent review (Yu et al., 2011).

A new generation of centrifugal devices has also emerged. As in total analysis systems, several operations can be integrated, including valving, siphoning, liquid mixing and volume metering and splitting. In centrifugal microfluidics devices, sometimes called LOD, all processes are controlled by centrifugal force by means of a rotating microstructured substrate to provide a pumping action for liquids (Ducree et al., 2007; Ríos et al., 2009; Mark et al., 2011). A centrifugal microfluidic device has been designed for solid sample preparation. This procedure could be extended as an alternative to large scale protocols for the dissolution of solid samples prior to analyses by other LOC or LOV protocols. It consists of a magnetically driven device, which allows the pulverization of solid samples by mechanical movement (Duford et al., 2009). In this work, a $0.1 \mathrm{~g}$ sample of potassium ferrocyanide was completely dissolved in $1.0 \mathrm{~mL}$ of water in $3 \mathrm{~s}$ while rotating at $1000 \mathrm{rpm}$. This system allows complete sample dissolution with up to $70 \%$ reduction in time with respect to static dissolution (Duford et al., 2009). The system also demonstrated the ability to pulverize a hard crystal, suggesting that a solid sample preparation step may be integrated on centrifugal microfluidic devices in the future.

\subsubsection{Organic Compound Analysis}

In this section, different miniaturized sample preparation procedures for the analysis of organic compounds are described. A wide range of organic pollutants, drugs and naturally-occurring compounds have been determined in a variety of matrices, 
including food and feedstuffs, biological and environmental solid samples. Table 7.3 shows selected applications of miniaturized sample preparation for organic compounds.

\subsubsection{Minimal Treatment-based Techniques}

In certain cases, very simple sample treatments such as dilution, dissolution or centrifugation can be carried out for the preparation of samples. In those cases, miniaturization has been overcome through important reductions in the volume of solvents used. Furthermore, additional automation of these procedures and direct solid analysis by different instrumental (sometimes miniaturized) techniques is also possible.

SS can be considered as a very simple alternative for sample preparation of solids. In the case organic compounds are analyzed, the solid sample is dissolved in organic solvents such as tetrahydrofuran, dimethylformamide, toluene, chlorinated solvents, alcohol-based solutions or even water, depending on the characteristics of the analyte (Self, 2005; Pawliszyn \& Lord, 2010). Volumes of solvents do not exceed $10 \mathrm{~mL}$, especially in the miniaturized format.

In some works, ultrasound irradiation has been applied to facilitate and improve sample dispersion since slurry homogeneity is an essential parameter affecting the sample representativeness. In this regard, it has to be considered that all of the solid matrix is introduced in the analytical instrument, and therefore, significant background effects and poorer detection and quantification limits are obtained. Additional effects related to organic compound degradation caused by ultrasound irradiation must be also taken into account.

In some cases, direct solid analysis is possible and techniques such as nuclear magnetic resonance (NMR), near infrared spectrometry (NIR), Raman spectroscopy, ion mobility spectrometry (IMS), direct analysis in real time mass spectrometry (DART-MS), and atmospheric pressure ionization mass spectrometry (API-MS) have been also employed for determination of organic analytes (Garrigues \& De la Guardia, 2013). In the case of mass spectrometric detection, the ionization source has to be selected depending on the type of analyte and nature of the sample. For example, in the case of IMS, laser sources for ionization allow direct analysis of organic compounds in solid environmental matrices with improved sensitivity in relation with other sources. This is the case, for example, of the determination of PAHs in petroleum-derived products, or the detection of explosives and drugs in different solid matrices by laser source-based IMS, for which any sample preparation is required (Márquez-Sillero et al., 2011).

GC techniques have been used for direct solid analysis, in most cases using the headspace sampling mode. New advances in this field include the coupling of GCIMS in portable instruments for in situ detection of volatiles from solid samples such as plants (Reyes-Garcés et al., 2013). 


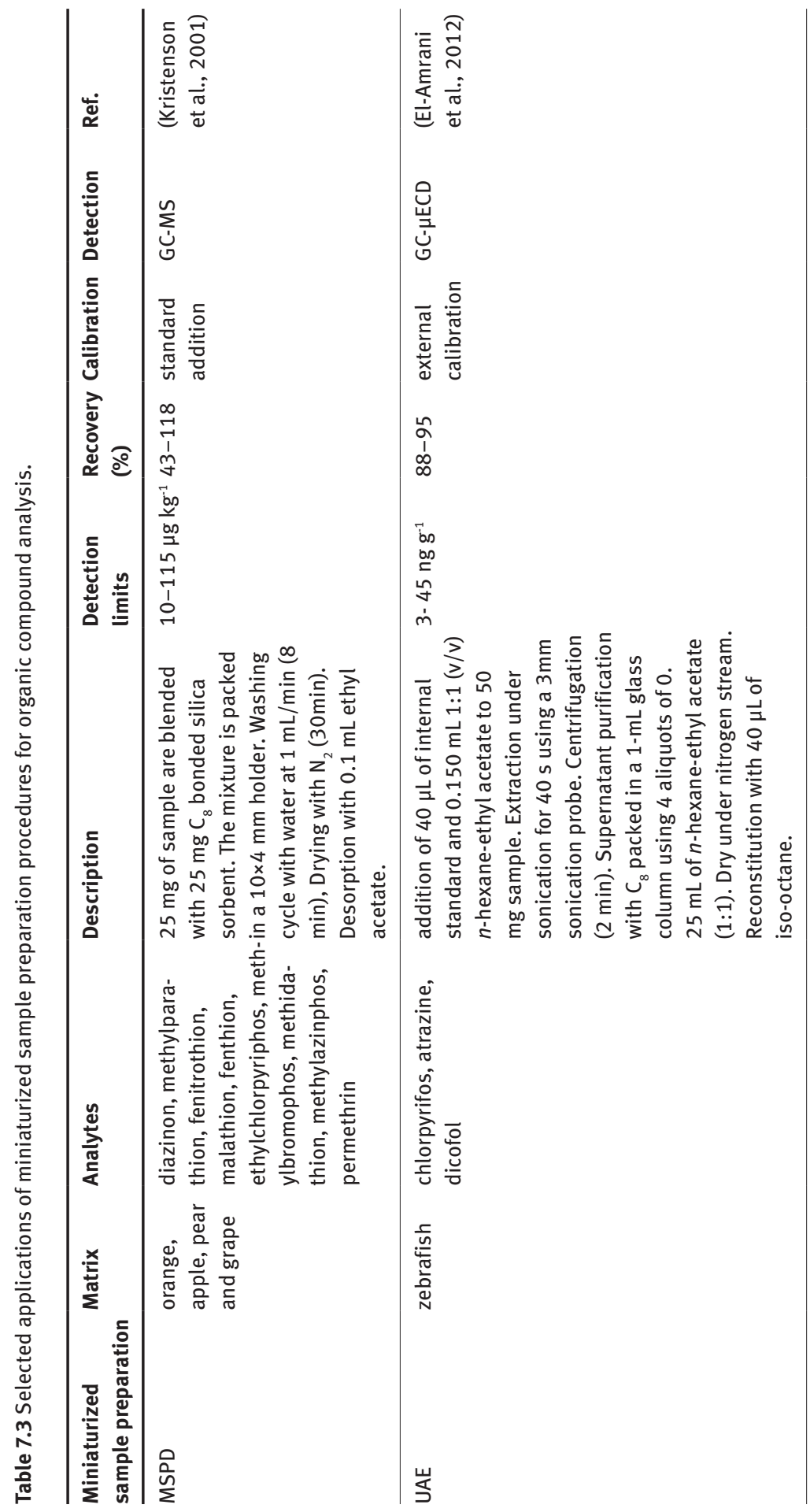




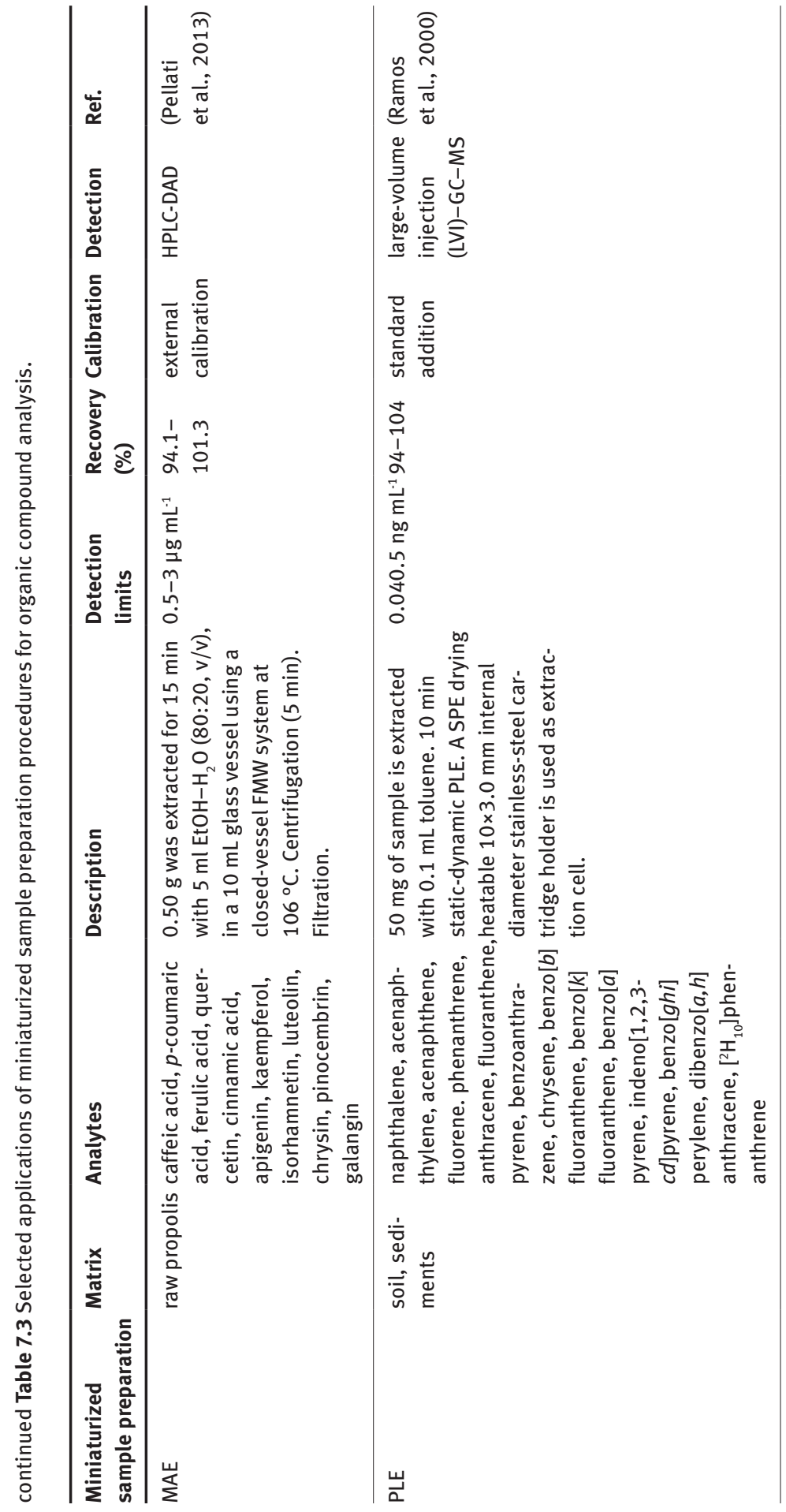




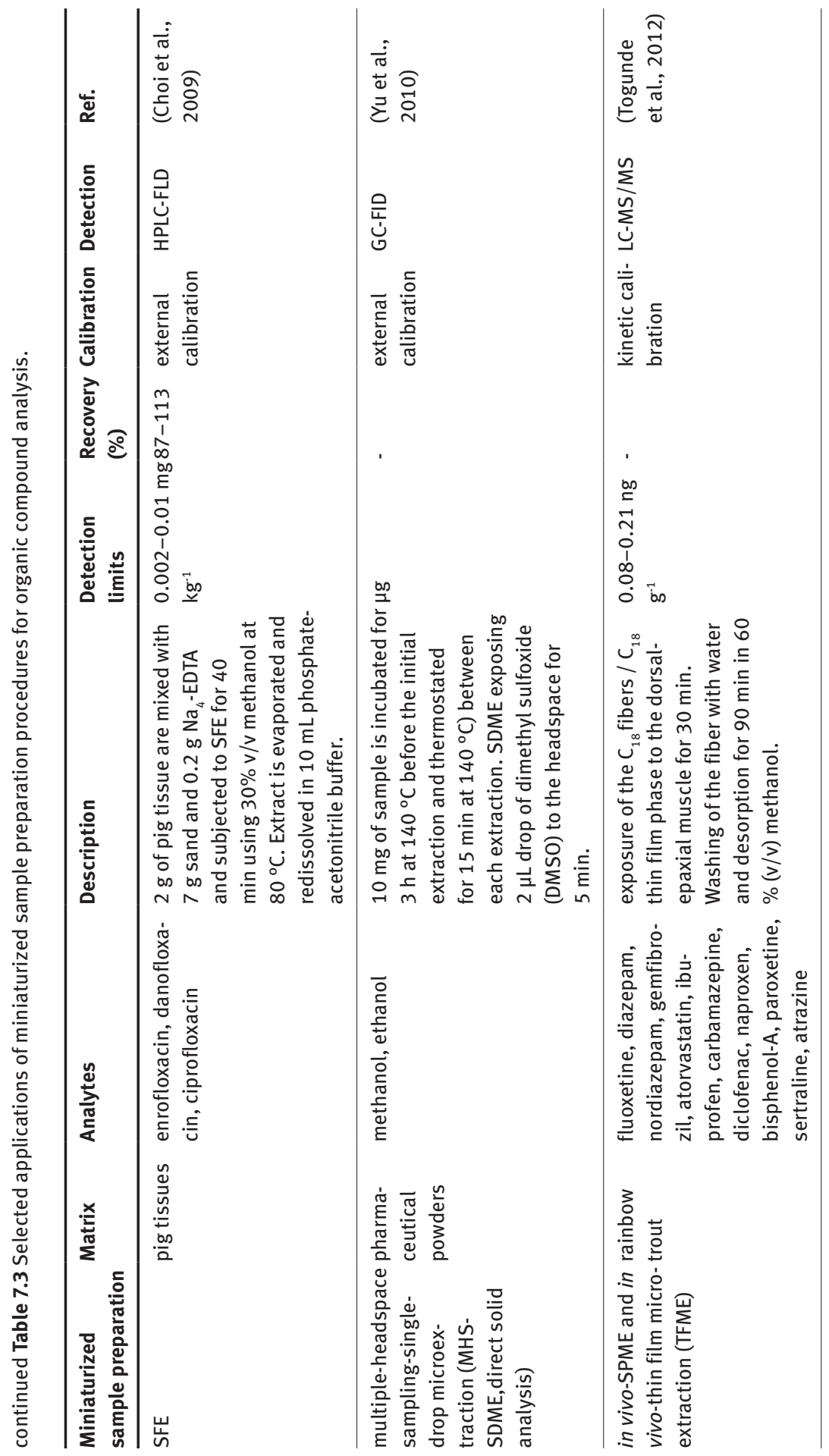




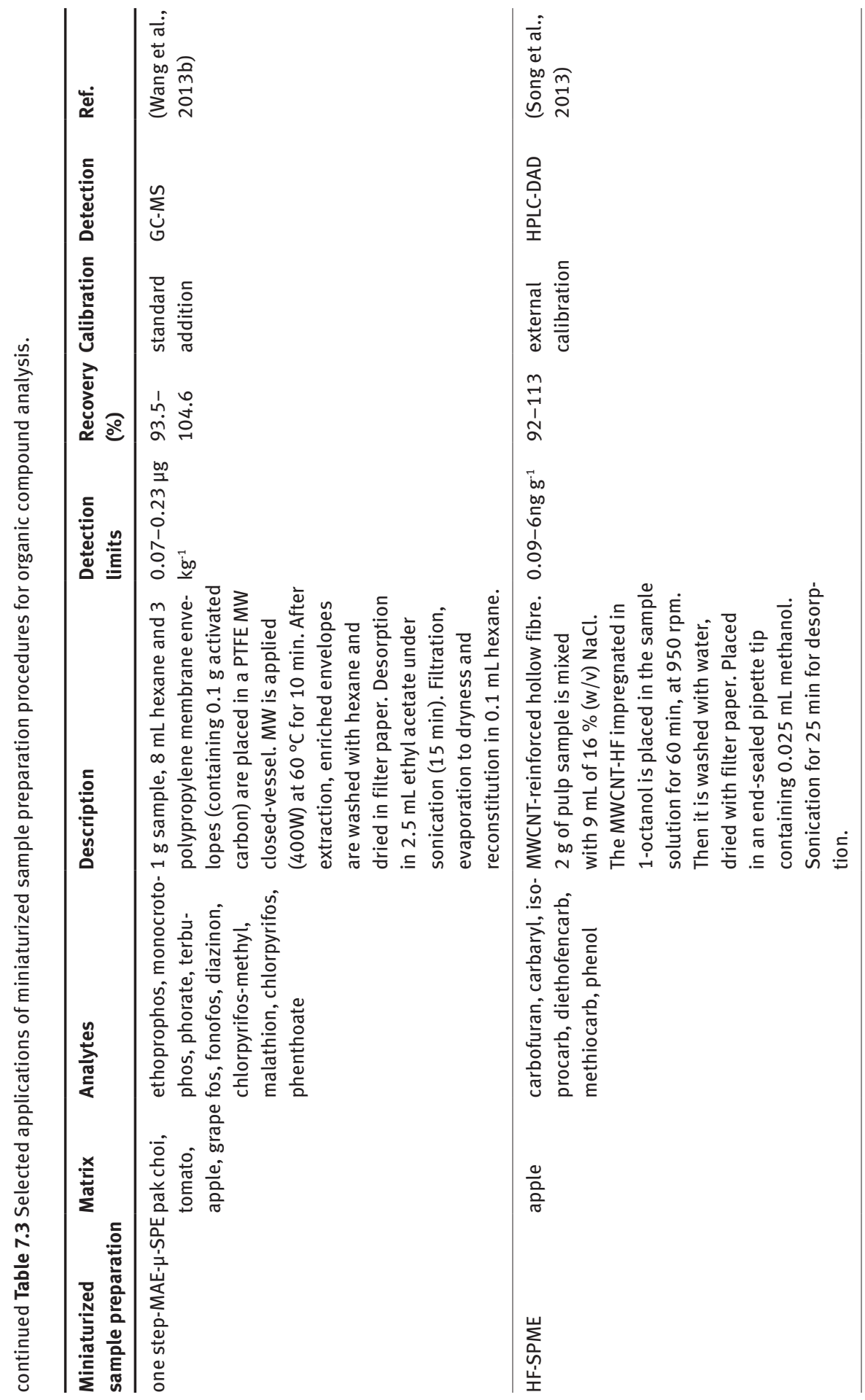




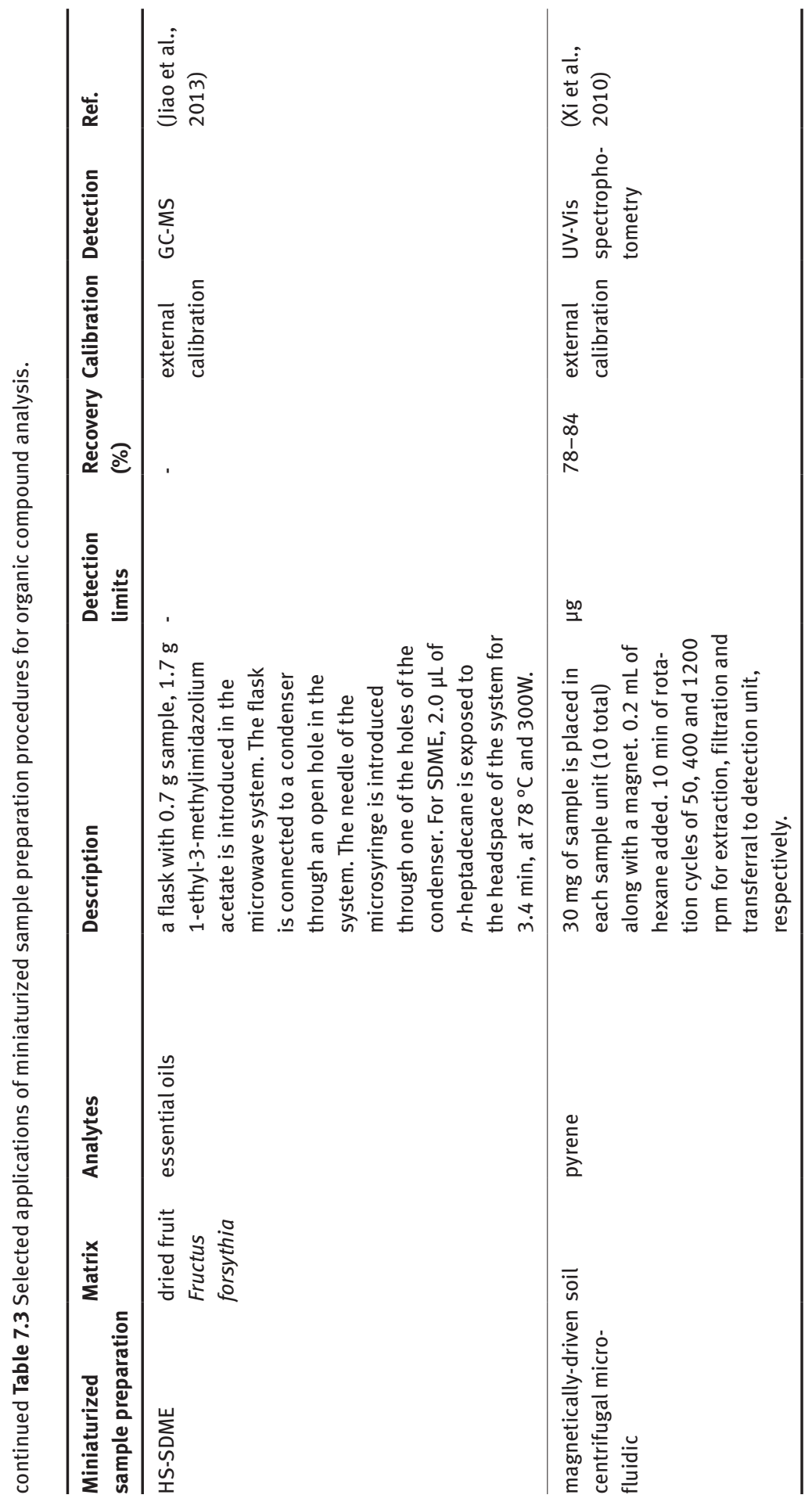


Improvements in solid sample analysis using these techniques has evolved through the development of miniaturized and, in some cases, hand-held devices. Electrochemical techniques, mainly coulometric- and voltammetric-based, have also been used for direct solid analysis, although to a lesser extent for organic compounds in comparison with inorganic analytes (Doménech-Carbó et al., 2013).

\subsubsection{Extraction-based Techniques}

Extraction-based techniques are frequently used for the analysis of organic compounds since analytes of interest can be directly extracted from the solid sample with no previous sample preparation step. In this sense, conventional and energy-assisted extractions, namely SFE, ASE, MAE or UAE, and MSPD have evolved towards their miniaturized modes or towards more environmentally friendly miniaturized alternatives such as LPME and SPME. In general, all of these techniques require a lower sample mass and/or reagent consumption. Experimentally, the volumes used can vary from few microliters to ten milliliters. In addition, low volume extraction vessels are required for accomplishing the solid sample preparation in miniaturized conditions. However, the implementation of new miniaturized procedures for the analysis of solid samples has been more limited in comparison with those for liquid samples (Ramos et al., 2005).

In most cases, extraction-based sample preparation techniques are essential tools for transferring analytes into a liquid phase that is suitable to be analyzed, or even suitable to be subjected to another separation/preconcentration sample preparation technique prior to analysis. In the vast majority of cases, separation-based techniques such as HPLC, GC or electrophoretic techniques coupled to different detectors are used.

\subsubsection{Direct Solid Treatment by Miniaturized Matrix Solid-phase Dispersion}

MSPD is one of the few extraction techniques that can be directly applied to solid, semi-solid and high-viscosity matrices. In fact, MSPD has been mainly used for the analysis of animal tissues, plants and foods, as well as environmental and human biological samples (Capriotti et al., 2013). A vast number of MSPD-based analytical methodologies have been developed for the determination of drugs, natural compounds and contaminants (Kristenson et al., 2001; Moliner-Martínez et al., 2009; Gutiérrez Valencia \& García de Llasera, 2011; Lu et al., 2011; Muñoz-Ortuño et al., 2012). However, publications concerning the use of MSPD for the determination of inorganic analytes are scarce (Capriotti et al., 2013).

In MSPD, the sample is homogeneously blended with a solid support (i.e., $\mathrm{C}_{8}, \mathrm{C}_{18}$, or florisil, among others, for the determination of organic compounds). The mixture is packed into an analytical column for further elution of the analytes. Miniaturized versions of this technique, in terms of reduced amounts of sample, inert support or elution solvent, have been successfully applied to the analysis of different samples, especially fatty biological tissues or environmental samples. Sample masses in the 
range $0.025-0.1 \mathrm{~g}$, inert support masses between $0.2-0.4 \mathrm{~g}$ and elution volumes between 0.1-3 mL have been used in miniaturized MSPD. Solvents such as acetonitrile, methanol, ethyl acetate, hexane, or their mixtures with other solvents in a much lower proportion, have primarily been used for elution.

For example, Kristenson et al. developed an automated miniaturized-MSPD using only $0.025 \mathrm{~g}$ of sample for the extraction of different pesticides from fruits, a $\mathrm{C}_{8}$ bonded silica sorbent and $0.1 \mathrm{~mL}$ ethyl acetate for elution. Reasonable recoveries from samples with a high reproducibility, automation and high sample throughput were emphasized by the authors (Kristenson et al., 2001). In another example, Muñoz-Ortuño et al. (2012) determined di(2-ethylhexyl) phthalate in fatty biological samples such as mussels following a miniaturized MSPD technique. $0.1 \mathrm{~g}$ of bivalve sample was mixed with only $0.4 \mathrm{~g}_{\text {of }} \mathrm{C}_{18}$ sorbent. Elution was carried out with $1.2 \mathrm{~mL}$ of acetonitrile.

The extraction of organic compounds can be challenging depending on the sample, as in the case of environmental solid samples. Strong interactions between the target molecule and the structure of the solid may lead to a more difficult extraction of the analyte from the matrix (Capriotti et al., 2013). In addition, miniaturizedMSPD may lead to matrix effects, such as the co-extraction of fatty acids in the case of biological tissues, thus necessitating different calibration strategies such as matrixmatching (Covaci et al., 2010) or more frequently, the standard addition method (Kristenson et al., 2001; Gutiérrez Valencia \& García de Llasera, 2011). In some cases, the use of an extra solid inert phase (co-sorbent) such as florisil allows for improved retention of the lipid phase of the sample, thus minimizing effects from matrix components (Moliner-Martinez et al., 2009; Gutiérrez Valencia \& García de Llasera, 2011; Lu et al., 2011; Muñoz-Ortuño et al., 2012).

In recent years, different sorbents have been introduced in miniaturized-MSPD to circumvent the potential lack of selectivity and matrix effects in the analyses. In this sense, innovations have been mainly introduced through the use of MIPs (Qiao \& Sun, 2010; Qiao \& Yan, 2010; Yan et al., 2011, 2012a, 2012b; Hong \& Chen, 2013). For example, Qiao and Yan et al. (2010) determined fluoroquinones and xanthine in serum using an MIP of ofloxacin and theophylline as a mixed template for selective recognition and extraction. Only $0.2 \mathrm{~g}$ of MIP particles along with $0.2 \mathrm{~g}$ of sample were used for MSPD and $4 \mathrm{~mL}$ acetonitrile-trifluoroacetic acid (99.5/0.5 (v/v)) for elution. Using standard addition to minimize potential matrix effects, recoveries were in the range of 90-104\% for all samples (Qiao \& Yan, 2010).

Although emerging novel sorbents have still found scarce application in miniaturized-MSPD, novel solid blending materials such as graphene or MWCNTs have also been used (Q. Liu et al., 2011; Su et al., 2011). For example, Liu et al. used graphene for the extraction of polybrominated diphenyl ethers (PBDEs) and their analogs from environmental samples. $0.1 \mathrm{~g}$ of sample was mixed with $0.01 \mathrm{~g}$ of graphene sorbent. $0.05 \mathrm{~g}$ of florisil was used as a co-sorbent and $1 \mathrm{~mL}$ hexane/dichloromethane or $1 \mathrm{~mL}$ acetone was used for elution. In spite of the enhancement in selectivity as a result of 
the capability of graphene to retain co-eluting matrix components, a co-sorbent was required to retain polar interferences and lipids. Standard addition was used to minimize matrix effects (Q. Liu et al., 2011).

New trends in miniaturized-MSPD are the use of energy-assisted procedures (e.g., ultrasound irradiation) or the combination with other clean-up steps. Recently, ultrasounds have been applied to sample preparation technique to improve EE as a result of improved contact between the solid adsorbent and the sample (Ramos et al., 2008; Karageorgou \& Samanidou, 2010, 2011; Rezaei \& Hosseini, 2011; Karageorgou et al., 2012, 2013, 2014; Barfi et al., 2013). In these cases, after mixing the sample and the sorbent, the mixture is transferred to a support (e.g., a syringe barrel or cartridge) and the extraction solvent is added. Both ends of the support are then closed, and the column is immersed in a sonicating system. Finally, the extract is collected from the support by gravity elution.

In the majority of these cases, ultrasonic baths are used to disperse the mixture for 5-30 min at a thermostated temperature of around $35-40{ }^{\circ} \mathrm{C}$. In the case of a highly effective extraction system for organophosphorus pesticides and triazines in fruits, a sonoreactor system was used which reduced the sonication time to only 1 min (Ramos et al., 2008).The application of ultrasounds requires one to take into account potential degradation of the target analytes. For example, Rezaei and Hosseini (2011) found that an increase in sonication time gave rise to degradation products of organochlorine pesticides.

Along with conventional MSPD sorbents such as $\mathrm{C}_{18}, \mathrm{C}_{8}$ or florisil, other inert blending materials such as commercial Oasis HLB sorbent or Nexus polymeric sorbent have been used when ultrasound-assisted miniaturized-MSPD is used (Ramos et al., 2008; Karageorgou \& Samanidou, 2010, 2011; Rezaei \& Hosseini, 2011; Karageorgou et al., 2012, 2013, 2014; Barfi et al., 2013;).

\subsection{Direct Solid Treatment by Miniaturized Ultrasound- and Microwave- assisted Extraction, Supercritical Fluid Extraction and Pressurized Liquid Extraction} Energy-assisted extractions (e.g., UAE, MAE, SFE and PLE) involving the transfer of analytes to an aqueous or organic phase have been widely used for the extraction of organic compounds from solid samples. Energy-assisted extractions not only can enhance analyte extraction, but also shorten extraction times. Miniaturization in these sample preparation techniques arise from a drastic reduction in the volumes used for extraction. However, scarce miniaturized applications aimed at the analysis of solids can be found in the literature.

Although UAE can be considered a well-established sample preparation technique, few ultrasound-assisted extractions in miniaturized format have been developed for the analysis of organic compounds. Exemplary applications have been devoted to the determination of PBDEs, PAHs, colistin and pesticides in different food, feedstuffs and environmental and biological tissue samples (Morales-Muñoz \& Luque de Castro, 2005; Aydin et al., 2006; Domeño et al., 2006; El-Amrani et al., 2012; 
Pena-Abaurrea et al., 2013a; Bizkarguenaga et al., 2014). The solvent-to-sample mass ratio is one of the most important parameters to take into account, apart from those related to the ultrasonic irradiation. Thus, the volumes of solvents have been reduced to $0.15-10 \mathrm{~mL}$, in comparison with traditional procedures which use up to $300 \mathrm{~mL}$ of solvents and no less than $1 \mathrm{~g}$ of sample for extraction (Bagherian et al., 2011). For example, El-Amrami et al. extracted chlorpyrifos, atrazine and dicofol from zebrafish using only $50 \mathrm{mg}$ of sample and $0.150 \mathrm{~mL}$ of hexane-ethyl acetate 1:1 (v/v). The low volumes allow shortened extraction times of just $40 \mathrm{~s}$ with good recoveries by using a high intensity sonication system with a $3 \mathrm{~mm}$ sonication probe (El-Amrani et al., 2012).

Although the use of conventional solvents such as hexane, ethyl acetate, methanol or acetone is common, new solvents have been also tested for miniaturized-format UAE. This includes natural deep eutectic solvents, which have the advantage of biodegradability and low toxicity. These solvents, formed by heating different alcohols and choline chloride at $80{ }^{\circ} \mathrm{C}$, have been used for the extraction of flavonoids from plants by ultrasound irradiation. For this purpose, $0.2 \mathrm{~g}$ of powdered plant tissue was mixed with just $2 \mathrm{~mL}$ of the eutectic solvent and subjected to ultrasound irradiation for $40 \mathrm{~min}$ (Bi et al., 2013).

The use of sonication probes not only allows reduced solvent consumption, but also enables automation (Morales-Muñoz \& Luque de Castro, 2005; Domeño et al., 2006). Morales-Muñoz \& Luque de Castro (2005) employed this approach for colistin analysis in animal feed by extracting $0.1 \mathrm{~g}$ of sample in a closed chamber with a flow injection manifold. A $1 \mathrm{~mm}$ sonication probe was inserted into the chamber for extraction using water as the solvent.

The incomplete removal of the matrix prompted different authors to consider the implementation of an extra clean-up step after miniaturized-UAE. As a result, coextracted compounds are eliminated prior to the analytical determination. SPE has been considered for this purpose as a suitable technique after miniaturized-UAE of different organic compounds (Aydin et al., 2006; El-Amrani et al., 2012; Pena-Abaurrea et al., 2013a).

Pena-Abaurrea et al. (2013a) achieved an extra clean-up by subjecting the extract to a disposable pipette SPE procedure. To this end, the low volume UAE extracted supernatant was taken with a micropipette into a $5 \mathrm{~mL}$ pipette tip containing $0.8 \mathrm{~g}$ acidic silica as a clean-up adsorbent to eliminate lipids from biological tissues for polychlorobiphenyls (PCB) determination.

A similar number of procedures based on MAE in miniaturized format (i.e., using less than $10 \mathrm{~mL}$ solvent volume) exist for the determination of organic compounds in solid matrices. Although plant and environmental materials have been mostly exensively analyzed, other applications for the quantification of contaminants in food can be also found in the literature. Volumes between 0.5 and $10 \mathrm{~mL}$ of organic extractants such as heptane, methanol, ethanol or ethyl acetate have been used for this purpose (Ramalhosa et al., 2012; Agudelo-Mesa et al., 2013; Azzouz \& Ballesteros, 2013; Pellati 
et al., 2013; Barrera-Vázquez et al., 2014; Song et al., 2014). Barrera-Vázquez et al. (2014) treated $0.3 \mathrm{~g}$ of sample extract, previously subjected to miniaturized-UAE, to miniaturized-MAE using $6 \mathrm{~mL}$ of ethyl acetate for $6 \mathrm{~min}$. This procedure showed higher EEs of anthraquinones from plants than the procedure using miniaturizedUAE alone.

As is the case for UAE-based procedures, co-extraction can be a common problem in procedures assisted by microwave irradiation. Several researchers have tried to circumvent this problem through the use of different strategies for calibration (Azzouz, 2012; Song, 2014), and/or applying a further extraction or clean-up step, either in miniaturized format or not (Azzouz \& Ballesteros, 2012; Barrera-Vázquez et al., 2014; Song et al., 2014). A different strategy was followed by Agudelo-Mesa et al. for determining aromatic amines in beef burgers. The authors used MAE as a clean-up technique for extraction (three consecutive extraction steps with heptane, $3 \mathrm{~min}$ in duration each). In this case, miniaturized-MAE was used to eliminate fatty material and soluble organic compounds from the matrix. Non-polar heterocyclic aromatic amines remaining in the settled phase were further solubilized in alkaline media to desorb the analytes from the matrix and to precipitate remaining proteins. A miniaturized preconcentration step based on DLLME was applied afterwards. After the whole procedure, however, matrix effects were found to affect the methodology, thus standard addition was used for calibration (Agudelo-Mesa et al., 2013).

The use of MW energy in closed vessels has also been reported not only to reduce extraction time, but also to reduce solvent volume in comparison with conventional maceration and heat reflux extraction for the extraction of plant-based materials. Phenolics and flavonoids have been extracted from plant materials using this approach (Pellati et al., 2013).

On the other hand, fewer publications concerning miniaturized versions of PLE for the analysis of solid samples are reported. The use of commercial PLE equipment limits the applicability of miniaturized formats, since these are usually developed for extractions in higher volume format. Solutions come through the use of smaller extraction cells or the construction of homemade miniaturized PLE equipment. Between 0.01-0.2 g of solid samples (foodstuffs, feedstuffs and soils) have been analyzed for to determine PCB, PBDE and PAH concentrations. Solvents used are typically hexane, dichloromethane or toluene, in volumes varying from only $0.1 \mathrm{~mL}$ to $6 \mathrm{~mL}$. As in the case of conventional PLE, both static and dynamic extraction steps are necessary for extraction (Crescenzi et al., 2000; Hyötyläinen et al., 2000; Ramos et al., 2000, 2007; Damm \& Kappe, 2011; Pena-Abaurrea et al., 2013b).

In most cases, miniaturized PLE is carried out by mixing the solid sample with an inert material such as silica or sand for retaining lipids or other interfering compounds, thus increasing the selectivity (Crescenzi et al., 2000; Ramos et al., 2007; Pena-Abaurrea et al., 2013b). For example, Pena-Abaurrea et al. developed an approach combining the use of a silica-based inert material and a homemade PLE device for improving selectivity in the extraction of PCBs and PBDEs from feedstuffs. 
The sample was mixed with $\mathrm{Na}_{2} \mathrm{SO}_{4}$ and acidified silica and placed in the extraction cell. For increased clean-up and selectivity, different layers of both acidic and neutral silica were also packed in the cell. Extraction was carried out in two static PLE cycles (7 min each) using $3 \mathrm{~mL}$ of hexane and hexane-dichloromethane and a final dynamic extraction (10 min) using $3 \mathrm{~mL}$ of hexane-dichloromethane (Pena-Abaurrea et al., 2013b).

Other relatively-miniaturized extractions based on the SFE have also found applications in the analysis of organic compounds in solid matrices (Pourmortazavi et al., 2014). These techniques have been used for the extraction of PAHs, pesticides and other organics from a variety of solids such as foods, diesel particulates, soils or biological and plant tissues (Starr \& Selim, 2008; Choi et al., 2009; Portet-Koltalo et al., 2009; Matsubara et al., 2012). Automation is possible when using this technique, and extraction in low volume cells $(2.5-10 \mathrm{~mL})$ can be achieved when an extra-miniaturization step is added (Matsubara et al., 2012). In most cases, a supercritical fluid is the only requirement for extraction, usually $\mathrm{CO}_{2}$ due to its greener analytical characteristics, with extraction times ranging from 20 to 150 min. Sample masses from 10-500 mg have been used. Sometimes, an organic modifier (i.e., methanol, ethanol, hexane, acetonitrile, etc.) is used to improve the extractability, especially for more polar analytes in complex environmental samples such as soils or sediments. After extraction, analytes can been collected in solvents (up to $10 \mathrm{~mL}$ ) that in most cases, but not always, are the same as that the organic modifier.

Due to the nature of solid samples, selective extraction is not always possible, thus leading in some cases to interferences. This is the case for lipids that are coextracted from biological tissues. In order to circumvent this potential problem, addition of solid materials such as florisil, silica gel or sand to the SFE extraction cells has been carried out. In a similar approach, Choi et al. added sand and $0.2 \mathrm{~g}$ of (ethylenedinitrile)-tetraacetic acid tetrasodium salt into $10 \mathrm{~mL}$ extraction cells for the SFE of fluoroquinones from $2 \mathrm{~g}$ pig tissues (Choi et al., 2009). Extraction was carried out using $30 \% \mathrm{v} / \mathrm{v}$ methanol as an organic modifier for $40 \mathrm{~min}$. The extraction of the most polar and hydrophilic compounds was significantly improved (87-113\% recovery) when the extra solids were added to the sample.

\subsection{Direct Solid Treatment by Microextraction Techniques}

Several examples can be found in literature in which solid samples are directly subjected to new generation miniaturized sample preparation techniques based on microextraction. However, it should be highlighted that only some formats of LPME and SPME are suitable for the direct analysis of solid samples with no previous treatment. This is the case of HS-SDME and DLLME modes, and both headspace and direct immersion-SPME.

Solid pharmaceuticals, plastics, vegetables and plants have been directly subjected to HS-SDME using sample masses in the range 0.01-5 g. Obviously, volatility of the selected analyte is a must, and therefore volatile residual organic solvents, volatile aldehydes, terpenes and styrenes have been selected as target compounds (Kim 
et al., 2005; Hansson \& Hakkarainen, 2006; Ligor \& Buszewski, 2008; Yu et al., 2010). In many cases, incubation at high temperatures $\left(60-150^{\circ} \mathrm{C}\right)$ is required to improve the transfer of the analyte to the headspace before HS-SDME (Kim et al., 2005; Hansson \& Hakkarainen, 2006; Yu et al., 2010). Solvents such as pentanol, butyl acetate or DMSO have been used for the extractions in volumes ranging from $0.5-2 \mu \mathrm{L}$.

Although HS-SDME is not an exhaustive sample preparation technique, complete direct extractions from solids can be achieved by exposing the extractant drop to the headspace during multiple consecutive cycles (Hansson \& Hakkarainen, 2006; Yu et al., 2010). Quantitative microextraction of residual solvents in pharmaceutical samples has been achieved following this technique. In this case, a long incubation step of $3 \mathrm{~h}$ at $140^{\circ} \mathrm{C}$ was initially required, as well as additional steps of thermostation $\left(15 \mathrm{~min}\right.$ at $140{ }^{\circ} \mathrm{C}$ ) between extractions in order to keep the headspace saturated with the analyte prior to extraction (Yu et al., 2010).

Owing to the difficulty of directly analyzing solids, only semi-solid samples have so far been directly subjected to DLLME procedures. Thus, formaldehyde and triclosan have been directly quantified in cosmetics following this sample preparation technique using only $20-80 \mu \mathrm{L}$ of dichloromethane and ionic liquids, respectively, for microextraction (Lavilla et al., 2010; Cabaleiro et al., 2011). The nature of cosmetic samples, which often contain high amounts of surfactants, makes a disperser solvent unnecessary, thus reducing the amount of reagents required.

SPME-based approaches have accounted for many more applications in the direct analysis of solid samples. The wide applicability of direct SPME methods lies in the fact that the volume of sample does not influence the extraction, when the sample volume is much higher than the volume of the SPME fiber (Müller, 1999). As such, applications for environmental, plant and biota analysis are numerous, although some other applications to the analysis of pharmaceuticals and packagings can be found (X. Zhang et al., 2012; Souza Silva et al., 2013; Zhu et al., 2013). Detection of antioxidant and aromatic compounds in plants, pharmaceuticals in living biological systems and volatiles in packaging are just some examples of applications of SPME to solid samples (Bicchi et al., 2000; Sides et al., 2001; Baták et al., 2003; Carrillo \& Tena, 2006; Togunde et al., 2012). In these cases, the SPME procedures typically consist of the direct insertion of the fiber into the living organism (in vivo-SPME) or, alternatively, exposing the SPME fiber to the headspace of a vial containing the solid sample (HS-SPME), which makes this technique completely free of extractant solvents. In the first case, biocompatibility of the fiber is essential in order to avoid adverse effects when sampling is performed with live animals. In this regard, PDMS, polypyrrole and $\mathrm{C}_{18}$ commercial fibers have been used with success. In the case of plant materials, being biocompatible is not a critical issue, thus PDMS-based fibers (PDMS, PDMS/ Divinylbenzene (DVB), PDMS/Carboxen (CAR)), or polyacrylate (PA) fibers have been mostly used. For example, trace pharmaceuticals such as gemfibrozil, atorvastatin, ibuprofen, carbamazepine, diclofenac, naproxen, bisphenol A, paroxetine and sertraline have been determined in live marine organisms such as rainbow trout and 
fathead minnow. In these experiments, $\mathrm{C}_{18}$ fibers were directly inserted into the dorsal-epaxial muscle of the fish, and microextraction was carried out over $30 \mathrm{~min}$. Acceptable reproducibility (4-22\% relative standard deviation, RSD) was achieved (Togunde et al., 2012).

HS-SPME mode has demonstrated its usefulness for the analysis of diverse volatile compounds in plants, mostly for analyzing aromatic profiles and scents (Bicchi et al., 2000; Barták et al., 2003; Carrillo \& Tena, 2006; Beck et al., 2008; Stashenko et al., 2009), either in the static or dynamic mode. Given the variety of possible aromatic compounds in plants, Bicchi et al. compared the effectiveness of PDMS, PDMS/ DVB, Carbowax (CW)/DVB, CAR/PDMS and CAR/DVB/PDMS fibers for the HS-SPME analysis of aromatic organic analytes from rosemary, sage, thyme and valerian. For extraction, $0.6 \mathrm{~g}$ of each plant was thermostated at $60^{\circ} \mathrm{C}$ for $1 \mathrm{~h}$ in a closed vial prior to extraction. Vials were shaken for $10 \mathrm{~s}$ at $10 \mathrm{~min}$ intervals and the extraction was carried out for $60 \mathrm{~min}$. The authors concluded that, although the fiber selection is strongly influenced by the polarity and volatility of each aromatic organic compound of the plant, PDMS-based fibers combined with porous solids provided, in general, good results (Bicchi et al., 2000).

\subsubsection{Combined Techniques}

A wide range of miniaturized sample preparation approaches are applied to liquid samples and cannot be directly used, in general, for the analysis of organics in solid and semisolid samples. In spite of the important advances in sample preparation, basic sample preparation techniques (i.e., homogenization, extraction, etc.) are still required for converting the solid into a suitable physical state (mostly liquid) prior to new generation miniaturized sample preparation techniques. This previous step can be also miniaturized or not. As discussed in the case of inorganic analysis, miniaturized techniques such as $\mu$-SPE, SPME, LPME and micro-total analysis systems ( $\mu$-TAS), among others, are applied. These techniques are in general characterized not only by the low volumes of reagents (from $\mu \mathrm{L}$ to $\mathrm{nL}$ ) and liquid-state sample (from few $\mathrm{mL}$ to $\mathrm{nL}$ ) required, but also by a high degree of sample clean-up, and, in most cases, analyte preconcentration.

\subsection{Solid-phase Extraction in Miniaturized Format}

Different formats of miniaturized SPE have been developed in recent years. Since sorbents control the selectivity and EE, efforts have been focused in this area, using polymers, MIPs, zeolites or activated carbon, among others. Examples include the determination of PBDEs in soils using copper (II) isonicotinate-based sorbents, ochratoxin $\mathrm{A}$ in coffee and cereals using Zeolite Linde Type L sorbent, pesticides in tissues using HayeSep $\mathrm{A} / \mathrm{C}_{18}$ sorbents and dicyandiamide in milk using a functionalized magnetic polymer sorbent (Basheer et al., 2008; Lee et al., 2012; Zhou et al., 2012; Chen et al., 2014). 
In most cases, miniaturized formats of SPE have evolved towards procedures based on the so called $\mu$-SPE. This mode is based on the use of a porous polypropylene membrane that protects the adsorbent from direct contact with the solid matrix, thus minimizing matrix effects. In general, the envelope-like polypropylene membrane containing the sorbent (around 20-50 mg) has dimensions of 1-2 cm in length and $0.5-1 \mathrm{~cm}$ in width. In general, analytes are first dissolved or solvent-extracted from the solid sample prior to be subjected to the $\mu$-SPE techniques.

Poly(methacrylic acid-ethylene glycol dimethacrylate) has been used for the extraction of sulfamerazine, sulfamethazine, sulfathiazole and sulfadiazine from chicken muscle. In order to transfer the analytes to a liquid phase, $1 \mathrm{~g}$ of sample was first extracted with $10 \mathrm{~mL}$ acetonitrile $10 \%$ (v/v). For $\mu$-SPE, just $20 \mathrm{mg}$ of sorbent in the polypropylene membrane were used for the extraction of $10 \mathrm{~mL}$ sample solution for 30 min under sonication. As in conventional SPE, steps of sorbent conditioning, extraction, washing and desorption are required. In this case, desorption was carried out with $0.4 \mathrm{~mL}$ of acetone containing $1 \%$ (v/v) ammonia under sonication for $30 \mathrm{~min}$ (Huang et al., 2012).

Interestingly, energy-assisted $\mu$-SPE procedures are common and ultrasound or microwave irradiation is frequently applied simultaneously to enhance the extraction (Basheer et al., 2008; Huang et al., 2012, 2013; Wang et al., 2013b). A novel approach based on miniaturized one-step MAE- $\mu$-SPE for the analysis of solid samples was recently reported. Wang et al. introduced three envelope-like polypropylene membranes containing $20 \mathrm{mg}$ of activated carbon into a PTFE microwave vessel which had been previously filled with $1 \mathrm{~g}$ of vegetable sample dissolved in $8 \mathrm{~mL}$ hexane. Extraction was carried out for $10 \mathrm{~min}$ at $400 \mathrm{~W}$ and $60{ }^{\circ} \mathrm{C}$. Good recoveries (94-105\%) and reproducibility was obtained (Wang et al., 2013b).

Another miniaturized-SPE format developed for solid sample analysis is pipette tip SPE which has been applied to the analysis of pesticides, picrosides, toxins or contaminants in plant materials and other biological tissues. $0.1-0.2 \mathrm{~mL}$ volume pipette tips containing 2-60 mg of sorbent are commonly used. As in other modes, different sorbents can be selected depending on the characteristics of the analyte. Conventional sorbents such as styrene divinylbenzene, $\mathrm{C}_{18}$ and graphene can be used. As the sample has to be aspirated into liquid form, a previous solid sample pre-treatment technique is required. Solvent extraction with organic solvents such as acetonitrile (Guan et al., 2010) or, in many cases, UAE with alcohol-based solvents (Pena-Abaurrea et al., 2013a ; Shen et al., 2013a, 2013b) has been reported. In addition, direct analysis of herbal powders has also been carried out (Wang et al., 2014). In this format, the extraction recovery is critically influenced by the number of aspirating and dispensing cycles. In one study, 25 consecutive aspirating/dispensing cycles were used for the extraction of pricosides from plants using a conventional $\mathrm{C}_{18}$ sorbent packed into a $0.2 \mathrm{~mL}$ pipette tip (Shen et al., 2013a). 


\subsection{Solid-phase Microextraction}

SPME techniques have found extensive use in the analysis of solid samples such as biological tissues, food, pharmaceuticals, plants and environmental samples and a vast number of different analytes have been determined, including organic contaminants, pesticides, herbicides and biologically active molecules. Except for those cases of direct solid analysis discussed in previous sections, samples have to be adapted to a liquid state containing the target analytes. Many different modes such as direct immersion-SPME, HS-SPME, in-tube-SPME, microextraction in a packed syringe or stir bar sorptive extraction (SBSE) have been used, all of them based on adsorption phenomena and characterized by minimal consumption of solvents, which in many cases are not required for extraction.

As in other techniques, solid samples must first be treated following procedures such as dissolution or homogenization in water (Domeño et al., 2005; Bali-Prasad et al., 2010) or organic solvents as acetone, acetonitrile or methanol. Other techniques for adapting the solid sample to a liquid state prior to SPME include extraction procedures such as miniaturized-MSPD (Campíns-Falcó et al., 2008; Moliner-Martinez et al., 2009), UAE (Ke et al., 2014), MAE (Ramil-Criado et al., 2004), SPE (Matin et al., 2013), subcritical water extraction (Hawthorne et al., 2000) or SFE (W. Liu et al., 2011).

Apart from the classical PDMS fiber coating, PA fibers, commercial mixed coatings such as PDMS/CAR, PDMS- DVB, CW/DVB and CW/templated resin, other solid sorbent phases based on antibodies, aptamers, polymers, nanoparticulated or carbon-based phases can be used in different SPME-based modes. In fact, new technologies come not only through the development of new SPME-based configurations, but also through the implementation of new adsorbent phases. In all cases, the polarity of the analyte determines the nature and characteristics of the sorbent used. In the case of direct immersion-SPME and HS-SPME, typical fiber lengths are in the range of $1-2 \mathrm{~cm}$. Fiber exposure times ranging from just a few min up to $60 \mathrm{~min}$ may be required for extractions. The use of high temperatures is also possible for speeding up the microextraction process, although this is more common in headspace mode.

Examples of SPME-based methods for the analysis of organics in solid phase samples can be found in the literature, such as the extraction of hydrocarbons in environmental samples (Matin et al., 2013), microextraction in packed syringe of clenbuterol from pork samples (Du et al., 2014), rotating disk sorptive extraction of PAHs from soils and sediments, (Hawthorne et al., 2000), SBSE of biologically active compounds from biological tissues and feed samples (Xu et al., 2010) or the evaluation of biotic exposure to contaminants by thin-film microextraction (Meloche et al., 2009).

The robustness of the supporting fiber and SPME coatings is a much more critical parameter when placed in direct contact with the sample (Mehdinia \& Aziz-Zanjani, 2013). In order to circumvent potential problems related to fiber robustness and carryover effects, novel strategies based not only on the development of more robust fibers, but also on new reinforced ones can be found in the literature (Ahmadi-Golsefidi et al., 2012; Song et al., 2013). The reinforcement of a hollow fiber with CNTs and 
MIPs combined with CNTs are emerging trends. For example, Song et al. developed a CNT-reinforced hollow fiber for the SPME of pesticides from apples. CNTs were immobilized in the pores of the hollow fiber and used for selective extraction of carbofuran, carbaryl, isoporcarb, diethofencarb and methiocarb. At the same time, the hollow fiber provided an extra sample clean-up step, since it was in direct contact with the sample. The only sample pre-treatment required was solubilization in $9 \mathrm{~mL}$ of $16 \%$ (v/v) NaCl aqueous solution (Song et al., 2013).

Extractions are usually carried out with stirring and the time needed may be reduced by as adding energy in the form of microwaves. Several one-step microwaveassisted sorbent-based microextraction procedures have been developed in this regard and a few of them have been applied to analyze solid samples such as oysters, sediments and wood by SPME (Pino et al., 2007; Wu \& Ding, 2010; Wu et al., 2012). In these cases, samples are only dispersed in water, and HS-SPME is carried out by inserting the needle through the top of the microwave system, exposing it to the headspace of the vial containing the slurried sample. The assistance of MW energy allowed equilibrium to be reached in only $5 \mathrm{~min}$ with good extraction recoveries. To the best of our knowledge no ultrasound-assisted-SPME based procedures have been published so far.

\subsection{Liquid-phase Microextraction}

A vast number of LPME techniques as SDME, HF-LPME, DLLME and their different modes have also been applied for the analysis of organic compounds in solid samples such as soils, foodstuffs, pharmaceuticals, biological tissues, plant materials, plastics and textiles (Pena-Pereira et al., 2010c; Chao et al., 2013; Pirsaheb et al., 2013; Viñas et al., 2013; Caballo et al., 2014; Lu \& Zhu, 2014). However, applications to solid sample analysis by LPME-based techniques only represent around $10 \%$ of the total (Kokosa et al., 2009).

Unlike SPME-based methods, all LPME modes require solvents for extractions, although at very low volumes varying from 0.5 to $100 \mu \mathrm{L}$, the highest volumes being required in the solidified organic droplet microextraction and DLLME modes and the lower volume in SDME-based modes.

Organic solvents and ionic liquids are commonly used to extract solid samples, depending mainly on the polarity of the organic analyte. In recent years, new aqueous extraction solvents have been gradually introduced, including aqueous cyclodextrins, surfactant-based extractants, coacervates and supramolecular solvents. Extraction times can vary from only $30 \mathrm{~s}$ to 40 min depending on the LPME technique and mode used. As in many other sample preparation techniques, temperature, ultrasound and microwave irradiation and vortexing have been used to acheive faster kinetics and to improve microextraction effectiveness from the solid matrix (Zhang \& Shi, 2010; Wang et al., 2012; Guo \& Kee Lee, 2013; Jiao et al., 2013; Lana et al., 2013; Abu-Bakar et al., 2014). For example, microwave energy has been used for the distillation and simultaneous HS-SDME of essential oils from the dried fruit Fructus forsythia. $0.7 \mathrm{~g}$ of sample, 
placed in a reaction flask containing $1.7 \mathrm{~g}$ of 1-ethyl-3-methylimidazolium acetate, was introduced in the cavity of the microwave apparatus. The flask was connected to a condenser through an open hole in the upper part of the microwave system, and to a microsyringe. For microextraction, a $2.0 \mu \mathrm{L}$ drop of $n$-heptadecane was exposed to the headspace of the system for $3.4 \mathrm{~min}$, at $78^{\circ} \mathrm{C}$ and at an irradiation power of $300 \mathrm{~W}$. In this case, the solvent was chosen on the basis of high boiling point, good EE, and also non-absorption of microwave energy. Precision, expressed as RSD, was found to be about 8 \% (Jiao et al., 2013).

Again, the physical state of the sample, with the exceptions of those modes discussed previously, must meet the requirement of being liquid, and thus, different sample pre-treatment strategies are applied in the case of solid samples. More specifically, processes based on solubilization (Seidi et al., 2011), UAE (GonzálezCurbelo et al., 2013; Sereshti et al., 2014) and MAE have been mainly used (Campillo et al., 2011; Agudelo Mesa et al., 2013). Other pre-treatment techniques such as MSDP (Yan et al., 2011), PLE (Lu \& Zhu, 2013) or SFE (Naeeni et al., 2011) have been less used.

$\mathrm{Lu}$ and $\mathrm{Zhu}$ (2013) determined chlorobenzenes in polyester fabrics by pressurized hot water extraction followed by vortex-assisted liquid-liquid microextraction (VALLME). First, the low polar analytes were selectively extracted from a $2 \mathrm{~g}$ sample by a static cycle of extraction at $160{ }^{\circ} \mathrm{C}$ and 1500 psi for $10 \mathrm{~min}$ and using $20 \%$ (v/v) acetonitrile. A $5 \mathrm{~mL}$ aliquot of the diluted extract was subjected to VALLME using 60 $\mu \mathrm{L}$ of carbon tetrachloride. After vortexing for $3 \mathrm{~min}$ and centrifugation, the enriched sedimented phase was used for analysis. Sample recoveries were obtained in the range of $68-92 \%$.

As mentioned before, not all pre-treatment techniques applied before LPME are miniaturized. For example, in the extraction of organophosphorous pesticides from cereals and flour, $20 \mathrm{~mL}$ of acetonitrile was required for UAE ( $5 \mathrm{~min}$ ) portion of the analysis. After centrifugation and filtration of the supernatant, the UAE was repeated again. Finally, pesticides were microextracted and preconcentrated by HF-LPME using $20 \mu \mathrm{L}$ of 1-octanol for 45 min (González-Curbelo et al., 2013).

\subsection{Fully Miniaturized Analytical Systems}

The use of micro-total analysis systems ( $\mu$-TAS or LOC) is undoubtedly one of the most challenging and, at the same time, promising developments in the analysis of organic compounds. The integration of the entire analytical process into a single miniaturized system will provide a step forward towards total miniaturization, automation and greening of analytical chemistry. In this sense, the use of volumes of both sample and reagents in the pL-nL range be highlighted as one of its several advantages. Nevertheless, one of its biggest pitfalls still lies in the achievement of adequate selectivity and sensitivity when it comes to trace analysis in highly complicated samples such as solid matrices. In addition, channels of the miniaturized analytical systems are likely to clog as a result of the introduction of suspended particles from solids. 
In recent years, the interest in the possibilities of $\mu$-TAS for organic analysis has increased and is reflected in the growing number of $\mu$-TAS applications in analytical chemistry (Ríos \& Zougagh, 2013). However, publications of these systems to the analysis of solids as biological, clinical, environmental and food samples are still scarce (Ríos et al., 2012).

Applications of LOC to solid analysis found in the literature were performed on soils, tablets, vegetables, fruits, and infant formula powder. The target analytes were antioxidants, vitamins, isoflavones, zearalenone, folic acid or formaldehyde (Miró et al., 2006; Lee et al., 2008; Plata et al., 2008; Atalay et al., 2011). In almost all of these cases, the most critical and error-prone step of sample pre-treatment is still carried out 'off-chip'. In off-chip sample preparation, steps such as pulverization, extraction, dilution and filtration are commonly carried out, not necessarily miniaturized at the same scale as the chip. For example, conventional UAE and subsequent $\mathrm{SPE}$ with $\mathrm{C}_{18}$ sorbent and filtration have been employed for the analysis of food dyes in fish, noodles and pepper powders. The combination of two different clean-up extraction steps and filtration was required in order to obtain extracts clean enough to flow through the microfluidic channels of the proposed microfluidic system. Analyte preconcentration, separation and chronoamperometric detection was then carried out on-chip (Lee et al., 2008).

By incorporating in the miniaturized system three different channels of fieldamplified sample stacking (FASS), field-amplified sample injection (FASI) and micellar electrokinetic chromatography, Lee et al. achieved a 10800-times higher sensitivity compared with conventional micellar electrokinetic chromatography with electrochemical detection (MEKC-ED). In spite of the clean-up steps carried out for sample preparation, salts and organic compounds from the matrix were found to affect the electrical signal and electrophoretic migration of the analytes (Lee et al., 2008).

Without a doubt, $\mu$-TAS will expand in the coming years, with much research being done in the field of solid sample analysis (Ríos \& Zougagh, 2013) and new advances based on both miniaturization and automation are continually being published. Centrifugal microfluidic systems have been used recently for automated integration of extraction, filtration and detection in soil analysis (Duford et al., 2009; Xi et al., 2010). Only Xi et al. used a magnetically-driven centrifugal microfluidic system for the direct determination of pyrene in soils. A detailed scheme on the proposed system is depicted in Figure 7.7. $30 \mathrm{mg}$ of soil was placed in each of the ten extraction chambers containing a magnet and $0.2 \mathrm{~mL}$ of hexane was added. Each chamber comprised stages of magnetically driven SLE, filtration and spectrometric detection, respectively. By rotating the systems at different speeds, the different steps took place. Good extraction yields and reproducibility was obtained, along with a very important reduction in sample mass and extractant volume in comparison with conventional techniques (Xi et al., 2010).

The combination of miniaturized sample preparation techniques such as $\mu$-SPE or LPME with $\mu$-TAS are also promising, and the first steps in this direction have already 


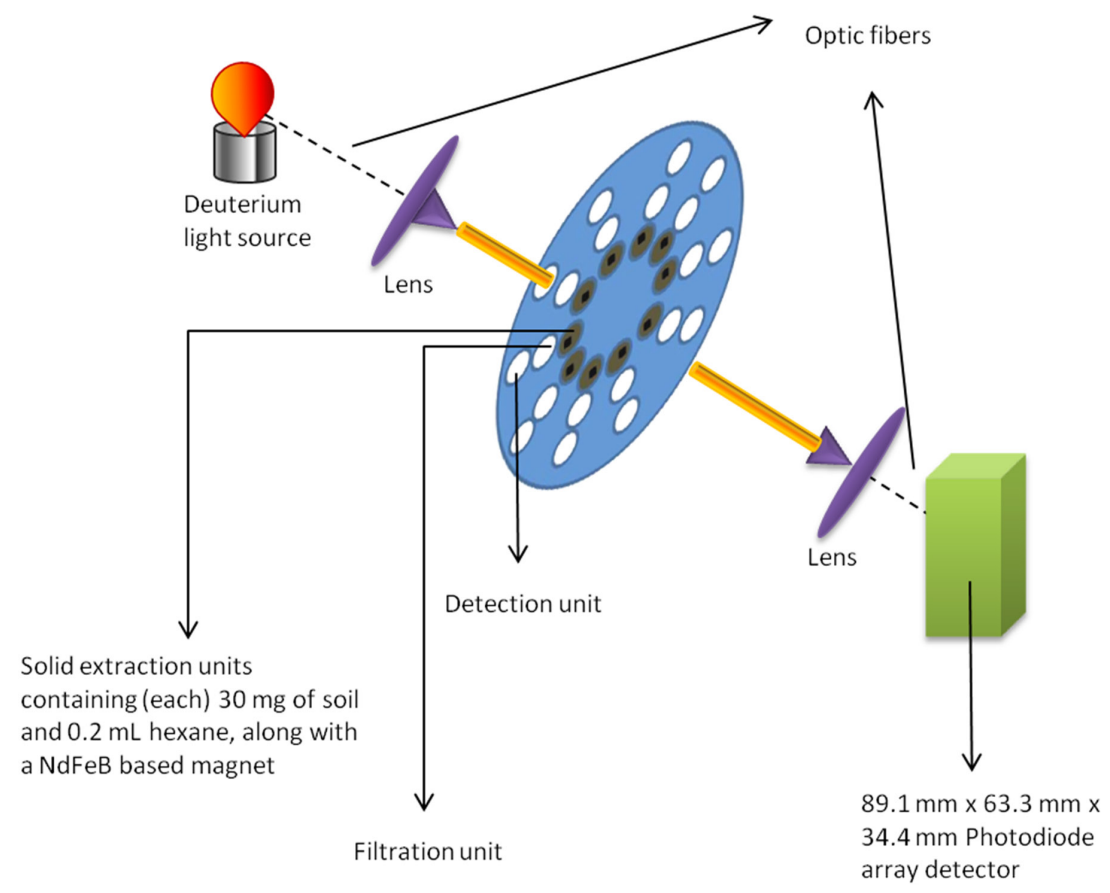

Figure 7.7 Schematic of a magnetically-driven centrifugal microfluidic system that includes automated integration of extraction, filtration and detection.

been achieved for the analysis of solid samples. For example, by combining HS-SDME with microfluidic chip electrophoresis, aliphatic amines have been determined in seafood. Sample pre-treatment was not completely eliminated however, since $0.5 \mathrm{~g}$ of sample was initially homogenized with $2 \mathrm{~mL}$ of aqueous buffer and $\mathrm{NaOH}$ prior to microextraction (Mark et al., 2011).

The LOV concept has also found application for the determination of organic compounds in solids. These miniaturized flow systems, which enable complete system automation and handling of volumes in the nanoliter range, are implemented using optical and electrochemical detection. Less frequently, separationbased instrumental detection techniques are used. However, important steps have already been taken in this direction with the aim of achieving analytical methodologies fully adapted to routine analysis laboratories, in which mainly chromatographic systems are used. In this regard, one of the bottlenecks lies in the hyphenation of the low sample flows with chromatographic systems and in potential column deterioration. Very recently, PCBs were quantified in solid waste leachates by coupling online LOV-BI and GC. In this system, PCBs were preconcentrated in the BI system (3 mg of commercial reversed-phase copolymeric sorbent) which served as an SPE- 
based device that allowed on-line sample pre-treatment and avoided further column damage. Injection of the different solutions was done using an automated multisyringe flow injection system. Even in these cases, sample pre-treatment is necessary and thus, previous filtration and dilution of samples are required. $4 \mathrm{~mL}$ of sample solution were finally used for analysis and only $80 \mu \mathrm{L}$ of ethyl acetate for elution. Recoveries in the range $87-116 \%$ were obtained. The authors also indicated a 25 -fold reduction of sorbent consumption with regard to traditional SPE procedures (Quintana et al., 2009).

\subsection{Future Trends}

Future trends in sample preparation should be oriented toward the increase of productivity through automation and the simultaneous direct treatment of several samples, but also considering the miniaturized procedures and the use of portable instrumentation for on-site determinations. In many cases, this will also mean the development of new interfaces to directly couple new sample preparation techniques with the detection unit of the portable instrumentation.

Research is ongoing to broaden the applications of virtually solventless techniques. The use of new extractant phases with improved sorption and robustness characteristics in the case of sorbent-based techniques (e.g., solid nanoparticle materials, graphene, PDMS-based modified coatings, magnetic nanocomposites and polymeric ionic liquids) is likely to improve extractions from highly complex solid matrices. Regarding LPME, new formats that are able to directly treat the solid sample and overcome potential matrix effects are required. The implementation of new extractant phases, based on nanoparticle materials, polymeric ionic liquids, or supramolecular solvents can also be anticipated.

Miniaturized microfluidic devices are very interesting for environmental and clinical monitoring. However, further developments are needed to link solid sample preparation to these miniaturized devices. In this sense, the implementation of new functionalized nanomaterials and new multifunctional particles (i.e., multifunctional protein particles encapsulating biologically active molecules such as enzymes and antibodies) within these techniques reveals promising routes for improving selectivity and sensitivity in the analysis of solid samples. This means the integration of all steps of the analytical process and avoiding off-chip sample preparation, especially in the case of solid samples.

Research efforts are also aimed at developing new sensors for on-site, real-time pollutant and biologically active molecule measurements. Special attention should be paid to the robustness, selectivity and precision of these systems. Electrochemical detection is expected to increase its popularity for analytical microchips due to: i) high sensitivity and selectivity, ii) low cost and iii) the possibility of miniaturization, portability and design of disposable devices. 
Magnetic beads are suitable and have increasing applications in microfluidic devices. Thus, magnetic nano- and microparticles could be used in SPE microchips and LOV systems due to the easy manipulation (separation) of magnetic particles using magnets and the possibility of automation. Associated advantages such as sample clean-up, mixing, analyte adsorption, transport and separation will also be considered for boosting their analytical applications. The coupling and automation of miniaturized systems using magnetic beads with chromatographic and other separation-based instrumental systems is still a challenge, although steps have been taken in this direction.

The development of new materials and nanomaterials could help several extraction and microextraction approaches. Moreover, the use of new solvents, like ionic liquids, or eutectic solvents should be continued.

With regard to increasing sample throughput, more research should be focused, for example, on centrifugal devices (6-10 sample throughput), microextraction in packed sorbent (with 96-well plates in polypropylene tips) and similar techniques. In addition, there are commercially available ultrasonic dual-probe and multi-probe systems (containing several tips) that presumably will play an important role in upcoming analytical method development and applications.

To summarize, fast, cheap, easy, integrated and automated procedures will be the future of miniaturized solid sample preparation procedures.

\subsection{Conclusions}

In recent decades, the pressure to decrease the usage of chemical reagents in the analytical laboratory has increased due to their potential toxicity and environmental impacts. Furthermore, there is a growing tendency towards the reduction of sample size, the time of operation and associated costs, along with the reduction of toxic solvents and wasteful residues. Moreover, the risks for the operator and analyte losses or contamination should be avoided. Thus, miniaturization and improvement of sample preparation alternatives is a focus of research. In addition, the possibility of integrating several analytical steps into only one miniaturized step, automation of these procedures with the possibility of direct on-site solid sample preparation, and enhancement of sample throughput are other state-of-the-art branches of research.

As reviewed in this chapter, different procedures could be applied for the analysis of metals, organometallics and organic compounds. In addition to miniaturization, acceleration of sample preparation procedures from several hours to a few minutes or seconds is carried out by means of ultrasound and microwave energy, the use of supercritical fluids and solvents at elevated temperature and pressure. Furthermore, several small vials, microvessels and cells were specially designed for different procedures, such as ASE, VPMAD, VPAD, AD, MAD, and PLE involving low-volume sample preparation procedures derived from the conventional ones. Generally, low amounts 
of sample (e.g., a few mg), and reduced volumes of reagents (e.g., $\mu \mathrm{L}-\mathrm{mL}$ ) are used in these procedures, thus minimizing the generation of wastes or residues.

Some procedures can be applied directly to solid samples while in other cases sample digestion or extraction of analytes is required prior to sample preparation. In the last case, preconcentration of analytes, with an associated elimination of potential matrix interferences, is generally performed.

As for the required volume of reagents and solvents, it can be stated that different levels of miniaturization have been achieved. Thus, some developed approaches require 5-10 mL (e.g., MAE, ASE, $\mu$-SPE), others only $1 \mathrm{~mL}$ (e.g., SS, UAE, UAD, LVMAD), while the most miniaturized techniques (e.g., LPME, LOC, LOV, LOD) only require a few $\mu \mathrm{L}, \mathrm{nL}$ or $\mathrm{pL}$.

\section{Abbreviations}

$\begin{array}{ll}\mu \text {-FIA } & \text { microflow injection analysis } \\ \mu \text {-SPE } & \text { micro-solid-phase extraction } \\ \mu \text {-TAS } & \text { micro-total analysis systems } \\ \text { AD } & \text { acid digestion } \\ \text { API-MS } & \text { atmospheric pressure ionization mass spectrometry } \\ \text { ASE } & \text { accelerated solvent extraction } \\ \text { AsB } & \text { arsenobetaine } \\ \text { AsC } & \text { arsenocholine } \\ \text { BCR } & \text { Community Bureau of Reference (Brussels } \\ \text { BI } & \text { bead injection } \\ \text { CAR } & \text { Carboxen } \\ \text { CNTs } & \text { carbon nanotubes } \\ \text { CPA } & \text { cold plasma ashing } \\ \text { CRMs } & \text { certified reference materials } \\ \text { CVAAS } & \text { cold-vapor atomic absorption spectrometry } \\ \text { CW } & \text { Carbowax } \\ \text { DART-MS } & \text { direct analysis in real time mass spectrometry } \\ \text { DDTC } & \text { diethyldithiocarbamate } \\ \text { DLLME } & \text { dispersive liquid-liquid microextraction } \\ \text { DMA } & \text { dimethylarsinic acid } \\ \text { DMSPE } & \text { dispersive micro solid-phase extraction } \\ \text { DMSO } & \text { dimethyl sulfoxide } \\ \text { DSS } & \text { direct solid sampling } \\ \text { DTE } & \text { dithioerythritol } \\ \text { DTT } & \text { dithiothreitol } \\ \text { DVB } & \text { divinylbenzene } \\ \text { DPX } & \text { disposable pipette extraction } \\ & \end{array}$




\begin{tabular}{|c|c|}
\hline EDTA & ethylenediaminetetraacetic acid \\
\hline $\mathrm{EE}$ & extraction efficiency \\
\hline $\mathrm{EF}$ & enrichment factor \\
\hline EPS & enzymatic probe sonication \\
\hline ETAAS & electrothermal atomic absorption spectrometry \\
\hline ETV & electrothermal vaporization \\
\hline ETV-ICP-OES & $\begin{array}{l}\text { electrothermal vaporization with inductively-coupled plasma optical } \\
\text { emission spectrometry }\end{array}$ \\
\hline ETV-ICP-MS & $\begin{array}{l}\text { electrothermal vaporization with inductively coupled plasma mass } \\
\text { spectrometry }\end{array}$ \\
\hline FAAS & flame atomic absorption spectrometry \\
\hline FASS & field-amplified sample stacking \\
\hline FASI & field-amplified sample injection \\
\hline FMW & focused microwave \\
\hline GC & gas chromatography \\
\hline GC-FID & gas chromatography flame ionization detector \\
\hline GC-MS & gas chromatography mass spectrometry \\
\hline $\mathrm{GC}-\mu \mathrm{ECD}$ & gas chromatography with micro electron capture detector \\
\hline HF-LPME & hollow-fiber liquid-phase microextraction \\
\hline HG-AAS & hydride generation atomic absorption spectrometry \\
\hline HG-AFS & hydride generation atomic fluorescence spectrometry \\
\hline HG-ICP-MS & $\begin{array}{l}\text { hydride generation coupled to inductively coupled plasma mass spec- } \\
\text { trometry }\end{array}$ \\
\hline HPHTSE & high-pressure, high temperature solvent extraction \\
\hline HPLC-DAD & high pressure liquid chromatography diode array detection \\
\hline HPLC-FLD & high pressure liquid chromatography with fluorimetric detector \\
\hline HPLC-HG-AFS & $\begin{array}{l}\text { high pressure liquid chromatography hydride generation atomic fluo- } \\
\text { rescence spectrometry }\end{array}$ \\
\hline HPLC-ICP-MS & $\begin{array}{l}\text { high pressure liquid chromatography coupled to inductively coupled } \\
\text { plasma mass spectrometry }\end{array}$ \\
\hline HPLC-MS & high pressure liquid chromatography mass spectrometry detection \\
\hline HPLC-UV & high pressure liquid chromatography ultraviolet detection \\
\hline HPSE & high-pressure solvent extraction \\
\hline HR-CS-ETAAS & $\begin{array}{l}\text { high resolution continuum source electrothermal atomic absorption } \\
\text { spectrometry }\end{array}$ \\
\hline HS-SDME & headspace single-drop microextraction \\
\hline HS-SPME & headspace solid-phase microextraction \\
\hline ICP-OES & inductively-coupled plasma optical emission spectrometry \\
\hline ICP-MS & inductively coupled plasma mass spectrometry \\
\hline IR & infrared radiation \\
\hline IMS & ion mobility spectrometry \\
\hline LA & laser ablation \\
\hline
\end{tabular}




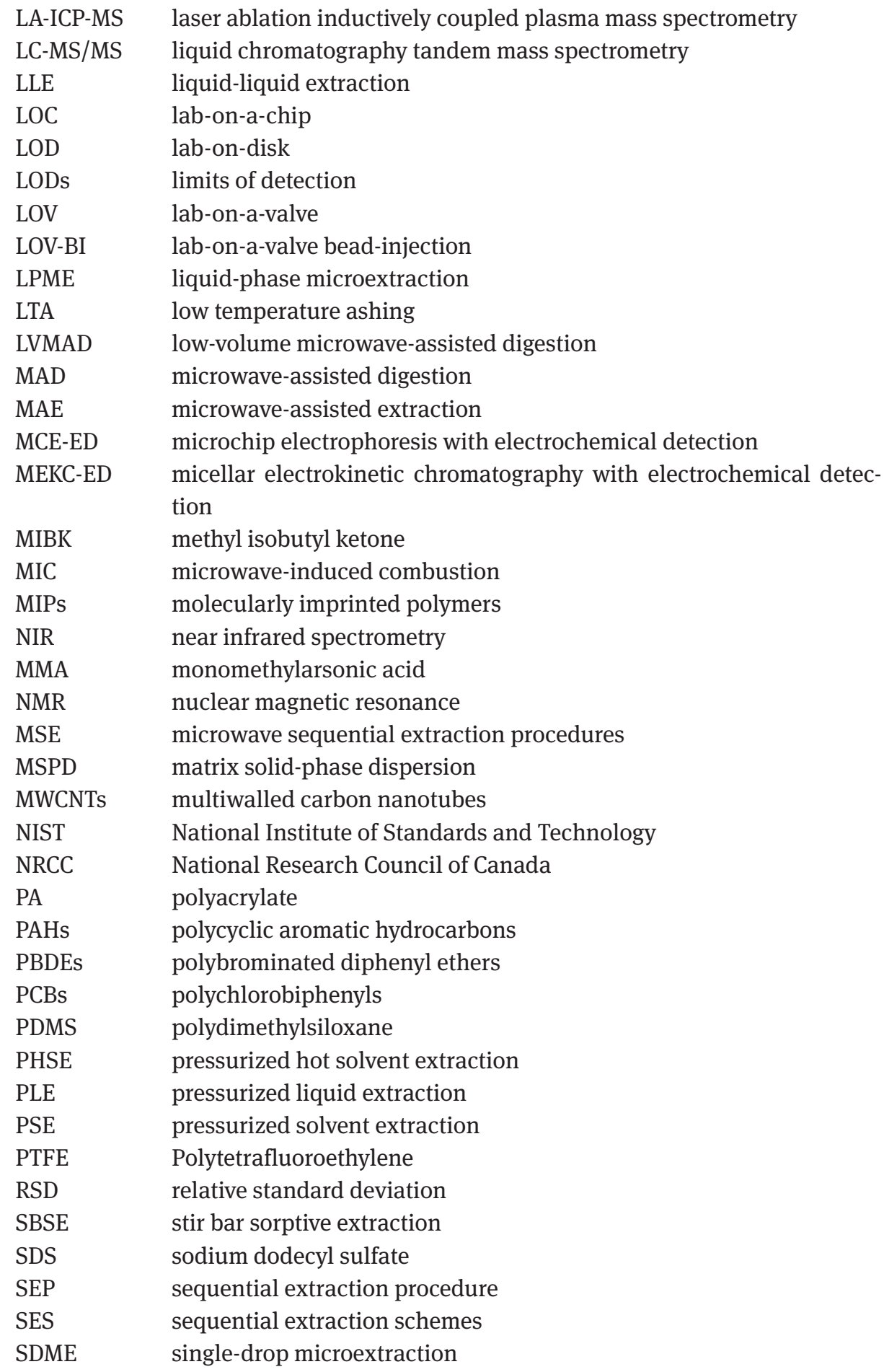




$\begin{array}{ll}\text { SFE } & \text { supercritical fluid extraction } \\ \text { SLE } & \text { solid-liquid extraction } \\ \text { SM\&T } & \text { Standards Measurements and Testing program from the European } \\ & \text { Commission } \\ \text { SPE } & \text { solid-phase extraction } \\ \text { SPME } & \text { solid-phase microextraction } \\ \text { SS } & \text { slurry sampling } \\ \text { SSE } & \text { subcritical solvent extraction } \\ \text { TMAH } & \text { tetramethyl ammonium hydroxide } \\ \text { Tris } & \text { tris(hydroxymethyl)aminomethane } \\ \text { TXRF } & \text { total reflection X-ray fluorescence spectrometry } \\ \text { UAD } & \text { ultrasound-assisted digestion } \\ \text { UAE } & \text { ultrasound-assisted extraction } \\ \text { USS } & \text { ultrasonic slurry sampling } \\ \text { USAEME } & \text { ultrasound-assisted emulsification microextraction } \\ \text { USEPA } & \text { United States Environmental Protection Agency } \\ \text { VALLME } & \text { vortex-assisted liquid-liquid microextraction } \\ \text { VPAD } & \text { vapor-phase acid digestion } \\ \text { VPMAD } & \text { vapor-phase microwave-assisted digestion } \\ \text { XRF } & \text { X-ray fluorescence spectrometry. }\end{array}$

\section{Acknowledgements}

I. de la Calle thanks Xunta de Galicia for financial support as a post-doctoral researcher of the I2C program and cofinancing by the European Social Fundings P.P. $0000421 \mathrm{~S}$ 140.08 .

\section{References}

Abu-Bakar, N., Makahleh, A. \& Saad, B. (2014). Vortex-assisted liquid-liquid microextraction coupled with high performance liquid chromatography for the determination of furfurals and patulin in fruit juices. Talanta, 120, 47-54.

Abu-Samra, A., Morris, J. S. \& Koirtyohann, S. R. (1975). Wet ashing of some biological samples in a microwave oven. Analytical Chemistry, 47, 1475-1477.

Acid digestion of sediments, sludges and soils; Method 3050B (1996). Retrieved March, 11, 2014 from http://www.epa.gov/sw-846/pdfs/3050b.pdf.

Adeyemo, D. J., Umar, I. M., Thomas, S. A. \& Agbaji, E. B. (2004). Analysis of lubricating oil for Cu, Fe, $\mathrm{Mn}, \mathrm{Ni}$ and $\mathrm{Zn}$ using electrothermal atomic absorption spectrometry. Instrumentation Science \& Technology, 32, 689-694.

Afridi, H. I., Kazi, T. G., Arain, M. B., Jamali, M. K., Kazi, G. H. \& Jalbani, N. (2007). Determination of cadmium and lead in biological samples by three ultrasonic-based samples treatment 
procedures followed by electrothermal atomic absorption spectrometry. Journal of $A O A C$ International, 90, 470-478.

Agudelo-Mesa, L. B., Padró, J. M. \& Reta, M. (2013). Analysis of non-polar heterocyclic aromatic amines in beefburguers by using microwave-assisted extraction and dispersive liquid-ionic liquid microextraction. Food Chemistry, 141, 1694-1701.

Ahmadi-Golsefidi, M., Es'haghi, Z. \& Sarafraz-Yazdi, A. (2012). Design, synthesis and evaluation of a molecularly imprinted polymer for hollow fiber-solid phase microextraction of chlorogenic acid in medicinal plants. Journal of Chromatography A, 1229, 24-29.

Akinlua, A., Torto, N. \& McCrindle, R. I. (2013). A new approach to sample preparation for the determination of trace metals in petroleum source rocks. Analytical Methods, 5, 4929-4934.

Alonso-Castillo, M. L., Vereda-Alonso, E., Siles-Cordero, M. T., Cano-Pavón J. M. \& García de Torres, A. (2011). Fractionation of heavy metals in sediment by using microwave assisted sequential extraction procedure and determination by inductively coupled plasma mass spectrometry. Microchemical Journal, 98, 234-239.

Al-Suhaimi, A. O. \& McCreedy, T. (2011). Microchip based sample treatment device interfaced with ICP-MS for the analysis of transition metals from environmental samples. Arabian Journal of Chemistry, 4, 195-203.

Alvarez, J., Marcó, L. M., Arroyo, J., Greaves, E. D. \& Rivas, R. (2003). Determination of calcium, potassium, manganese, iron, copper and zinc levels in representative samples of two onion cultivars using total reflection X-ray fluorescence and ultrasound extraction procedure. Spectrochimica Acta Part B: Atomic Spectroscopy, 58, 2183-2189.

Alzaga, R. Díez, S. \& Bayona, J. M. (2003). Supercritical fluid extraction. In Z. Mester \& R. Sturgeon (Eds.), Sample preparation for trace element analysis (pp. 313-341). The Netherlands: Elsevier.

Amarasiriwardena, D. Krushevska, A., Argentine, M. \& Barnes, R. M. (1994). Vapour-phase acid digestion of micro samples of biological material in a high-temperature, high-pressure asher for inductively coupled plasma atomic emission spectrometry. The Analyst, 119, 1017-1021.

Amberger, M. A. \& Broekaert, J. A. C. (2010). Direct multielement determination of trace elements in boron carbide powders by slurry sampling ETV-ICP-OES. Journal of Analytical Atomic Spectrometry, 25, 1308-1315.

Amoedo, L., Capelo, J. L., Lavilla, I. \& Bendicho, C. (1999). Ultrasound-assisted extraction of lead from solid samples: a new perspective on the slurry-based sample preparation methods for electrothermal atomic absorption spectrometry. Journal of Analytical Atomic Spectrometry, 14, 1221-1226.

Antes, F. G., Duarte, F. A., Mesko, M. F., Nunes, M. A. G., Pereira, V. A., Müller, E. I., Dressler, V. L. \& Flores, E. M. M. (2010). Determination of toxic elements in coal by ICP-MS after digestion using microwave-induced combustión. Talanta, 83, 364-369.

Arain, M. B., Kazi, T. G., Jamali, M. K., Jalbani, N., Afridi, H. I., Sarfraz, R. A. \& Shah, A. Q. (2007). Determination of toxic elements in muscle tissues of five fish species using ultrasound-assisted pseudodigestion by electrothermal atomic absorption spectrophotometry: optimization study. Spectroscopy Letters, 40, 861-878.

Arain, M. B., Kazi, T. G., Jamali, M. K., Jalbani, N., Afridi, H. I. \& Baig, J. A. (2008). Speciation of heavy metals in sediment by conventional, ultrasound and microwave assisted single extraction methods: A comparison with modified sequential extraction procedure, Journal of Hazardous Materials, 154, 998-1006.

Araújo, G. C. L., Nogueira, A. R. A. \& Nóbrega, J. A. (2004). Microwave single vessel acid-vapor extraction: effect of experimental parameters on $\mathrm{Co}$ and Fe determination in biological samples. Microchimica Acta, 144, 81-85.

Araújo, G. C. L., Nogueira, A. R. A. \& Nóbrega, J. A., (2000). Single vessel procedure for acid-vapour partial digestion in a focused microwave: Fe and Co determination in biological samples by ETAAS. The Analyst, 125, 1861-1864. 
Arroyo, L., Trejos, T., Gardinali, P. R. \& Almirall, J. R. (2009). Optimization and validation of a laser ablation inductively coupled plasma mass spectrometry method for the routine analysis of soils and sediments. Spectrochimica Acta Part B: Atomic Spectroscopy, 64, 16-25.

Arruda, S. C. C., Rodriguez, A. P. M. \& Arruda, M. A. Z. (2003). Ultrasound-assisted extraction of Ca, K and Mg from In Vitro Citrus culture. Journal of the Brazilian Chemical Society, 14, 470-474.

Associação Brasileira de Cimento Portland, Manual Técnico 4, Procedimentos para análises químicas de cimentos e suas matérias-primas, 2003 (134 pp) ABCP.

Atalay, Y. T., Vermeir, S., Witters, D., Vergauwe, N., Verbruggen, B., Verboven, P., Nicolaï B. M. \& Lammertyn, J. (2011). Microfluidic analytical systems for food analysis. Trends in Food Science and Technology, 22, 386-404.

Aydin, M. E., Tor, A. \& Özcan, S. (2006). Determination of selected polychlorinated biphenyls in soil by miniaturised ultrasonic solvent extraction and gas chromatography-mass-selective detection. Analytica Chimica Acta, 577, 232-237.

Azzouz, A. \& Ballesteros, E. (2012). Combined microwave-assisted extraction and continuous solid-phase extraction prior to gas chromatography-mass spectrometry determination of pharmaceuticals, personal care products and hormones in soils, sediments and sludge. Science of Total Environment, 419, 208-215.

Bachmann, L., Zezell, D. M., Ribeiro, A. D., Gomes L. \& Ito, A.S. (2006). Fluorescence spectroscopy of biological tissues - A review. Applied Spectroscopy Reviews, 41, 575-590.

Bagherian, H., Ashtiani, F. Z., Fouladitajar, A. \& Mohtashamy, M. (2011). Comparisons between conventional, microwave- and ultrasound-assisted methods for extraction of pectin from grapefruit. Chemical Engineering and Processing: Process Intensification, 50, 1237-1243.

Balarama-Krishna, M. V. \& Arunachalam, J. (2004). Ultrasound-assisted extraction procedure for the fast estimation of major, minor and trace elements in lichen and mussel samples by ICP-MS and ICP-AES. Analytica Chimica Acta, 522, 179-187.

Balarama-Krishna, M. V., Ranjit, M., Karunasagar, D. \& Arunachalam, J. (2005). A rapid ultrasoundassisted thiourea extraction method for the determination of inorganic and methyl mercury in biological and environmental samples by CVAAS. Talanta, 67, 70-80.

Balarama-Krishna, M. V., Venkateswarlu, G., Thangavel, S. \& Karunasagar, D. (2013) Oxidative pyrolysis combined with the microwave-assisted extraction method for the multi-elemental analysis of boron carbide powders by inductively coupled plasma optical emission spectrometry (ICP-OES). Analytical Methods, 5, 1515-1523.

Baldwin, S., Deaker, M. \& Maher, W. (1994). Low-volume microwave digestion of marine biological tissues for the measurement of trace elements. The Analyst, 119, 1701-1704.

Bali Prasad, B., Prasad Tiwari, M., Madhuri, R. \& Sindhu, P. (2010). Enantioselective quantitative separation of $\mathrm{d}$ - and l-thyroxine by molecularly imprinted micro-solid phase extraction silver fiber coupled with complementary molecularly imprinted polymer-sensor. Journal of Chromatography A, 1217, 4255-4266.

Bangroo, P. N., Jagga, C. R., Arora, H. C. \& Rao, G. N. (1995). Spectrometric determination of wear metals and additive elements in lubricating oils by AAS and ICP-AES. Atomic Spectrosccopy, 16, 118-120.

Barbosa, A. F., Segatelli, M. G., Pereira, A. C., Santos, A. D., Kubota, L. T., Luccas, P. O. \& TeixeiraTarley, C. R. (2007). Solid-phase extraction system for Pb (II) ions enrichment based on multiwall carbon nanotubes coupled on-line to flame atomic absorption spectrometry. Talanta, 71, 1512-1519.

Barfi, V., Asghari, A., Rajabi, M., Barfi, A. \& Saeidi, I. (2013). Simplified miniaturized ultrasoundassisted matrix solid phase dispersion extraction and high performance liquid chromatographic determination of seven flavonoids in citrus fruit juice and human fluid samples: Hesperetin and naringenin as biomarkers. Journal of Chromatography A, 1311, 30-40.

Barker, S. A., (2000). Matrix solid-phase dispersion, Journal of Chromatography A, 885, 115-127. 
Barker, S. A., (2007). Matrix solid-phase dispersion (MSPD), Journal of Biochemical and Biophysical Methods, 70, 151-162.

Barrera-Vázquez, M. F., Comini, L. R., Martini, R. E., Núñez-Montoya, S. C., Bottini, S., Cabrera, J. L. (2014). Comparisons between conventional, ultrasound-assisted and microwave-assisted methods for extraction of anthraquinones from Heterophyllaea pustulata Hook f. (Rubiaceae). Ultrasonics Sonochemistry, 21, 478-484.

Barták, P., Bednar, P., Čáp L., Ondráková, L. \& Stránsky, Z. (2003). SPME - A valuable tool for investigation of flower scent. Journal of Separation Science, 26, 715-721.

Basheer, C., Narasimhan, K., Yin, M., Zhao, C., Choolani, M. \& Lee, H. K. (2008). Application of micro-solid-phase extraction for the determination of persistent organic pollutants in tissue samples. Journal of Chromatography A, 1186, 358-364.

Batista, B. L., Rodrigues, J. L., Souza, V. C. D. \& Barbosa Jr., F. (2009). A fast ultrasound-assisted extraction procedure for trace elements determination in hair samples by ICP-MS for forensic analysis. Forensic Science International, 192, 88-93.

Beck, J. J., Merrill, G. B., Palumbo, J. D. \& O'Keeffe, T. L. (2008). Strain of Fusariumoxysporum isolated from almond hulls produces styrene and 7-methyl-1,3,5-cyclooctatriene as the principal volatile components. Journal of Agricultural Food Chemistry, 56, 11392-11398.

Belarra, M. A., Resano, M., Vanhaecke, F. \& Moens, L. (2002). Direct solid sampling with electrothermal vaporization/atomization: what for and how? TrAC Trends in Analytical Chemistry, 21, 828-839.

Bellido-Milla, D., Ordaz-Garcia, S. M., Guerrero-Valiente, J. L. \& Hernández-Artiga, M. P. (2002). Rapid procedure to determine wear metals in lubricating oils and the analysis of variance in the evaluation of sample preparation procedures. Microchimica Acta, 138, 59-64.

Bendicho, C, Lavilla, I., Pena, F. \& Costas, M., (2011). Green sample preparation methods. In M. de la Guardia \& S. Garrigues (Eds.), Challenges in Green Analytical Chemistry (pp. 63-106). Cambridge: Royal Society of Chemistry.

Bendicho, C. \& de Loos-Vollebregt, M.T.C. (1991). Solid sampling in electrothermal atomic absorption spectrometry using commercial atomizers: A review. Journal of Analytical Atomic Spectrometry, 6, 353-374.

Bendicho, C., De La Calle, I., Pena, F., Costas, M., Cabaleiro, N. \& Lavilla, I. (2012a). Ultrasoundassisted pretreatment of solid samples in the context of green analytical chemistry. TrAC Trends in Analytical Chemistry, 31, 50-60.

Bendicho, C., Lavilla, I., Pena, F. \& Romero, V. (2012b). Green chemistry in analytical atomic spectrometry: a review. Journal Analytical Atomic Spectroscopy, 2012, 27, 1831-1857.

Bengtson, B. \& Lundholm M., (1988). Surface analysis with the glow discharge lamp. State of the art and prospects for future development. Journal of Analytical Atomic Spectroscopy, 3, 879-882.

Benson, W. H. Francis, P. C., Birge, W. J., Black, J. A. (1983). A simple method for acid extraction of Cd from fish eggs or fish tissues. Atomic Spectroscopy, 4, 212-213.

Bermejo, P., Capelo, J. L., Mota, A., Madrid, Y. \& Cámara, C. (2004). Enzymatic digestion and ultrasonication: A powerful combination in analytical chemistry. TrAC Trends in Analytical Chemistry, 23, 654-663.

Bermejo-Barrera, P., Fernández-Nocelo, S., Moreda-Piñeiro, A. \& Bermejo-Barrera, A. (1999). Usefulness of enzymatic hydrolysis procedures based on the use of pronase $E$ as sample pre-treatment for multi-element determination in biological materials. Journal of Analytical Atomic Spectroscopy, 14, 1893-1900.

Bermejo-Barrera, P., Muñiz-Naveiro, O., Moreda-Piñeiro, A. \& Bermejo-Barrera, A. (2000). Experimental designs in the optimisation of ultrasonic bath-acid-leaching procedures for the determination of trace elements in human hair samples by atomic absorption spectrometry. Forensic Science International, 107, 105-120. 
Bettiol, C., Stievano, L., Bertelle, M., Delfino, F. \& Argese, E., (2008). Evaluation of microwaveassisted acid extraction procedures for the determination of metal content and potential bioavailability in sediments. Applied Geochemistry, 23, 1140-1151.

Bi, W., Tian, M. \& Row, K. H. (2013). Evaluation of alcohol-based deep eutectic solvent in extraction and determination of flavonoids with response surface methodology optimization. Journal of Chromatography A, 1285, 22-30.

Bicchi, C., Drigo, S. \& Rubiolo, P. (2000). Influence of fibre coating in headspace solid-phase microextraction-gas chromatographic analysis of aromatic and medicinal plants. Journal of Chromatography A, 892, 469-485.

Bizkarguenaga, E., Iparragirre, A., Zabaleta, I., Vallejo, A., Fernández, L. A., Prieto, A. \& Zuloaga, 0. (2014). Focused ultrasound assisted extraction for the determination of PBDEs in vegetables and amended soil. Talanta, 119, 53-59.

Bocca, B., Lamazza, A., Pino, A., De Masi, E., lacomino, M., Mattei, D., Rahimi, S., Fiori, E., Schillaci, A., Alimonti, A. \& Forte, G. (2007). Determination of 30 elements in colorectal biopsies by sector field inductively coupled plasma mass spectrometry: method development and preliminary baseline levels. Rapid Communication in Mass Spectrometry, 21, 1776-1782.

Borges, A. R., Becker, E. M., Lequeux, C., Vale, M. G. R., Ferreira, S. L. C. \& Welz, B. (2011). Method development for the determination of cadmium in fertilizer samples using high-resolution continuum source graphite furnace atomic absorption spectrometry and slurry sampling. Spectrochimica Acta Part B: Atomic Spectroscopy, 66, 529-535.

Borgese, L., Zacco, A., Bontempi, E., Colombi, P., Bertuzzi, R., Ferretti, E., Tenini, S. \& Depero, L. E. (2009). Total reflection of X-ray fluorescence (TXRF): a mature technique for environmental chemical nanoscale metrology. Measurement Science and Technology, 20, 1-7.

Borgese, L., Zacco, A., Bontempi, E., Pellegatta, M., Vigna, L., Patrini, L., Riboldi, L., Rubino, F. M. \& Depero, L. E. (2010). Use of total reflection X-ray fluorescence (TXRF) for the evaluation of heavy metal poisoning due to the improper use of a traditional ayurvedic drug. Journal of Pharmaceutical and Biomedical Analysis, 52, 787-790.

Borkowska-Burnecka, J. (2000). Microwave assisted extraction for trace element analysis of plant materials by ICP-AES. Fresenius Journal of Anaytical Chemistry, 368, 633-637.

Brady, D. V., Montalvo Jr., J. G., Glowacki, G. \& Pisciotta, A. (1974). Direct determination of zinc in sea-bottom sediments by carbon tube atomic absorption spectrometry. Analytica Chimica Acta, $70,448-452$.

Brancalion, M. L., Aurelio, M. \& Arruda, Z. (2005). Evaluation of medicinal plant decomposition efficiency using microwave ovens and mini-vials for Cd determination by TS-FF-AAS. Microchimica Acta, 150, 283-290.

Bredendiek-Kämper, S., von Bohlen, A., Klockenkämper, R., Quentmeier, A. \& Klockow, D. (1996). Microanalysis of solid samples by laser ablation and total reflection X-ray fluorescence. Journal of Analytical Atomic Spectroscopy, 11, 537-541.

Brunori, C., Ipolyi, I., Macaluso, L. \& Morabito, R. (2004). Evaluation of an ultrasonic digestion procedure for total metal determination in sediment reference materials, Analytica Chimica Acta, 510, 101-107.

Burguera, J. L. \& Burguera, M. (2012). Analytical applications of emulsions and miroemulsions. Talanta, 96, 11-20.

Cabaleiro, N., Pena-Pereira, F., De La Calle, I., Bendicho, C., \& Lavilla, I. (2011). Determination of triclosan by cuvetteless UV-vis micro-spectrophotometry following simultaneous ultrasound assisted emulsification-microextraction with derivatization: Use of a micellar-ionic liquid as extractant. Microchemical Journal, 99, 246-251

Caballo, C., Sicilia, M. D. \& Rubio, S. (2014). Fast, simple and efficient supramolecular solventbased microextraction of mecoprop and dichlorprop in soils prior to their enantioselective determination by liquid chromatography-tandem mass spectrometry. Talanta, 119, 46-52. 
Caballo-López, A. \& Luque de Castro, M. D. (2007). Determination of cadmium in leaves by ultrasound-assisted extraction prior to hydride generation, pervaporation and atomic absorption detection. Talanta, 71, 2074-2079.

Cabañero, A. I., Madrid, Y. \& Cámara, C. (2005). Enzymatic probe sonication extraction of Se in animal-based food samples: a new perspective on sample preparation for total and Se speciation analysis. Analytical and Bioanalytical Chemistry, 381, 373-379.

Cal-Prieto, M. J., Felipe-Sotelo, M., Carlosena, A., Andrade, J. M., López-Mahía, P., Muniategui, S. \& Prada, D. (2002). Slurry sampling for direct analysis of solid materials by electrothermal atomic absorption spectrometry (ETAAS). A literature review from 1990 to 2000. Talanta, 56, 1-51.

Camel, V. (2000). Microwave-assisted solvent extraction of environmental samples. TrAC Trends in Analytical Chemistry. 19, 229-248.

Camel, V. (2003). Solid phase extraction of trace elements. Spectrochimica Acta Part B: Atomic Spectroscopy, 58, 1177-1233.

Campillo, N., Viñas, P., Martínez-Castillo, N. \& Hernández-Córdoba, M. (2011). Determination of volatile nitrosamines in meat products by microwave-assisted extraction and dispersive liquid-liquid microextraction coupled to gas chromatography-mass spectrometry. Journal of Chromatography A, 1218, 1815-1821.

Campíns-Falcó, P., Verdú-Andrés, J., Sevillano-Cabeza, A., Molins-Legua, C., Herráez-Hernández, R. (2008). New micromethod combining miniaturized matrix solid-phase dispersion and in-tube in-valve solid-phase microextraction for estimating polycyclic aromatic hydrocarbons in bivalves. Journal of Chromatography A, 1211, 13-21.

Canepari, S., Cardarelli, E., Ghighi, S. \& Scimonelli, L. (2005). Ultrasound and microwave-assisted extraction of metals from sediment: a comparison with the BCR procedure. Talanta, 66 , 1122-1130.

Capelo, J. L., Lavilla, I. \& Bendicho, C. (1998). Ultrasound-assisted extraction of cadmium from slurried biological samples for electrothermal atomic absorption spectrometry. Journal of Analytical Atomic Spectrometry, 13, 1285-1290.

Capelo, J. L., Lavilla, I. \& Bendicho, C. (1999). Solid-liquid extraction of copper from slurried samples using high intensity probe sonication for electrothermal atomic absorption spectrometry. Talanta, 50, 905-911.

Capelo, J. L., Maduro, C. \& Vilhena, C. (2005). Discussion of parameters associated with the ultrasonic solid-liquid extraction for elemental analysis (total content) by electrothermal atomic absorption spectrometry. An overview. Ultrasonics Sonochemistry, 12, 225-232.

Capelo, J. L., Ximénez-Embún, P., Madrid-Albarrán, Y. \& Cámara, C. (2004). Enzymatic probe sonication: Enhancement of pProtease-catalyzed hydrolysis of selenium bound to proteins in yeast. Analytical Chemistry, 76, 233-237.

Capitelli, F., Colao, F., Provenzano, M. R., Fantoni, R., Brunetti G. \& Senesi. N. (2002). Determination of heavy metals in soils by Laser induced breakdown spectroscopy. Geoderma, 106, 45-62.

Capriotti, A. L., Cavaliere, C., Giansanti, P., Gubbiotti, R., Samperi, R. \& Lagana, A. (2010). Recent developments in matrix solid-phase dispersion extraction. Journal of Chromatography A, 1217, 2521-2532.

Capriotti, A. L., Cavaliere, C., Lagana, A., Piovesana, S. \& Samperi, R. (2013). Recent trends in matrix solid-phase dispersion. TrAC Trends in Analytical Chemistry, 43, 53-66.

Carballo, S., Terán, J., Soto, R. M., Carlosena, A., Andrade, J. M. \& Prada, D. (2013). Green approaches to determine metals in lubricating oils by electrothermal atomic absorption spectrometry (ETAAS). Microchemical Journal, 108, 74-80.

Carballo-Paradelo, S., Soto-Ferreiro, R. M., Carlosena-Zubieta, A., Terán-Baamonde, J., AndradeGarda, J. M. \& Prada-Rodríguez, D. (2012). Pressurized liquid extraction to determine Mg, Al, Ti, $\mathrm{Cu}, \mathrm{Sn}$ and $\mathrm{Pb}$ in lubricating oils by inductively coupled plasma mass spectrometry. Journal of Analytical Atomic Spectrometry, 27, 1694-1700. 
Carpenter, R. C., (1981) The determination of cadmium, copper, lead and thallium in human liver and kidney tissue by flame atomic absorption spectrometry after enzymatic digestion. Analytica Chimica Acta, 125, 209-213.

Carpinteiro, J., Rodríguez, I. \& Cela, R. (2004). Applicability of solid-phase microextraction combined with gas chromatography atomic emission detection (GC-MIP AED) for the determination of butyltin compounds in sediment samples. Analytical and Bioanalytical Chemistry, 380, 853-857.

Carrillo, J. D. \& Tena, M. T. (2006). Determination of volatile compounds in antioxidant rosemary extracts by multiple headspace solid-phase microextraction and gas chromatography. Flavour and Fragrance Journal, 21, 626-633.

Carvalho-Santos, W. P., Teixeira-Castro, J., Almeida-Bezerra, M., Pires-Fernandes, A., Costa-Ferreira, S. L. \& Andrade-Korn, M. G. (2009). Application of multivariate optimization in the development of an ultrasound-assisted extraction procedure for multielemental determination in bean seeds samples using ICP OES. Microchemical Journal, 91, 153-158.

Chailapakul, O., Korsrisakul, S., Siangproh, W. \& Grudpan, K. (2008). Fast and simultaneous detection of heavy metals using a simple and reliable microchip-electrochemistry route: An alternative approach to food analysis. Talanta, 74, 683-689.

Chang, I.-H., Tulock, J. J., Liu, J., Kim, W.-S., Cannon Jr., D. M., Lu, Y., Bohn, P. W., Sweedler , J. V. \& Cropek, D. M. (2005). Miniaturized lead sensor based on lead-specific DNAzyme in a nanocapillary interconnected microfluidic device. Environmental Science \& Technology, 39, 3756-3761.

Chao, Y., Jian, Z., Tu, Y. \& Huang, Y. (2013). Rapid on-line microextraction of neutral analytes in plastic-bottled beverages through ultrasound-assisted push/pull perfusion hollow-fiber liquidliquid-liquid microextraction. Microchemical Journal, 110, 386-394.

Chen, S., Liu, C., Yang, M., Lu, D., Zhu, L. \& Wang, Z. (2009). Solid-phase extraction of Cu, Co and $\mathrm{Pb}$ on oxidized single-walled carbon nanotubes and their determination by inductively coupled plasma mass spectrometry. Journal of Hazardous Materials, 170, 247-251.

Chen, X. \& Marshall, W. D. (1999). Enzymatic digestion-high-pressure homogenization prior to slurry introduction graphite furnace atomic absorption spectrometry for selenium determination in plant and animal tissues. Journal of Agricultural and Food Chemistry, 47, 3727-3732.

Chen, X., Zhou, L., Zhao, Y., Pan, S. \& Jin, M. (2014). Application of nanoring amino-functionalized magnetic polymer dispersive micro-solid-phase extraction and ultrafast liquid chromatography-tandem mass spectrometry in dicyandiamide residue analysis of powdered milk. Talanta, 119, 187-192.

Chen, X.-W. \& Wang, J.-H. (2007). The miniaturization of bioanalytical assays and sample pretreatments by exploiting meso-fluidic lab-on-valve configurations: A review. Analytica Chimica Acta, 602, 173-180.

Choi, J. H., Mamun, M. I. R., Abd El-Aty, A. M., Kim, K. T., Koh, H. B., Shin, H. C., Kim, J., Lee, K. B. \& Shim, J. H. (2009). Inert matrix and $\mathrm{Na}_{4}$ EDTA improve the supercritical fluid extraction efficiency of fluoroquinolones for HPLC determination in pig tissues. Talanta, 78, 348-357.

Claisse F. (2003). Fusion and fluxes In Z. Mester \& R. Sturgeon (Eds.) Sample preparationn for trace element analysis, (pp. 301-312). The Netherlands: Elsevier.

Clement, R.-E. \& Hao, C. (2010). Sample preparation techniques for environmental organic pollutant analysis. In J. Pawliszyn \& H. L. Lord (Eds.) Handbook of sample preparation (pp. 249-265), Hoboken, N.J.: John Wiley \&Sons, Inc.

Colombini, V., Bancon-Montigny, C., Yang, L., Maxwell, P., Sturgeon, R. E. \& Mester, Z. (2004). Headspace single-drop microextraction for the detection of organotin compounds. Talanta, 63 , 555-560.

Costas, M., Lavilla, I., Gil, S., Pena, F., de la Calle, I., Cabaleiro, N. \& Bendicho, C. (2010). Evaluation of ultrasound-assisted extraction as sample pre-treatment for quantitative determination 
of rare earth elements in marine biological tissues by inductively coupled plasma-mass spectrometry. Analytica Chimica Acta, 679, 49-55.

Covaci, A., Mashiya, J. G., Molope, I., Tshiame, I., Molatlhegi, R., Ngobeni, P. (2010). Miniaturized method based on matrix solid-phase dispersion for the rapid screening of 36 pesticides in agricultural food commodities. Analytical Letters, 43, 1400-1410.

Crescenzi, C., Di Corcia, A., Nazzari, M. \& Samperi, R. (2000). Hot phosphate-buffered water extraction coupled on-line with liquid chromatography/mass spectrometry for analyzing contaminants in soil. Analytical Chemistry, 72, 3050-3055.

Cucheval, A. \& Chow, R.C.Y. (2008). A study on the emulsification of oil by power ultrasound. Ultrasonics Sonochemistry, 15, 916-920.

Czégény, Z., Berente, B., Óvári, M., García-Tapia, M. \& Záray, G. (1998). Microwave-assisted vapor-phase acid digestion of cellulose nitrate filters for elemental analysis of airborne dust samples. Microchemical Journal, 59, 100-106.

Damm, M. \& Kappe, C. O. (2011). A high-throughput platform for low-volume high-temperature/ pressure sealed vessel solvent extractions. Analytica Chimica Acta, 707, 76-83.

Dantas, A. N. S., Matos, W. O., Gouveia, S. T. \& Lopes, G. S. (2013). The combination of infrared and microwave radiation to quantify trace elements inorganic samples by ICP-OES. Talanta, 107, 292-296.

Davidson, C. M. \& Delevoye, G. (2001). Effect of ultrasonic agitation on the release of copper, iron, manganese and zinc from soil and sediment using the BCR three-stage sequential extraction. Journal of Environmental Monitoring, 3, 398-403.

De Jesus, A., Silva, M. M. \& Vale, M. G. R. (2008). The use of microemulsion for determination of sodium and potassium in biodiesel by flame atomic absorption spectrometry. Talanta, 74 , 1378-1384.

De Jesus, R. M., Silva, L. O. B., Castro, J. T., de Azevedo-Neto, A. D., De Jesus, R. M. \& Ferreira, S. L. C. (2013). Determination of mercury in phosphate fertilizers by cold vapor atomic absorption spectrometry. Talanta, 106, 293-297.

De la Calle, I., Cabaleiro, N., Costas, M., Pena, F., Gil, S., Lavilla, I. \& Bendicho, C. (2011a) Ultrasound-assisted extraction of gold and silver from environmental samples using different extractants followed by electrothermal-atomic absorption spectrometry. Microchemical Journal, 97, 93-100.

De la Calle, I., Cabaleiro, N., Lavilla, I. \& Bendicho, C. (2009). Analytical evaluation of a cup-horn sonoreactor used for ultrasound-assisted extraction of trace metals from troublesome matrices. Spectrochimica Acta Part B: Atomic Spectroscopy, 64, 874-883.

De la Calle, I., Cabaleiro, N., Lavilla, I. \& Bendicho, C. (2013a). Ultrasound-assisted single extraction tests for rapid assessment of metal extractability from soils by total reflection X-ray fluorescence. Journal of Hazardous Materials, 260, 202- 209.

De la Calle, I., Cabaleiro, N., Romero, V., Lavilla, I. \& Bendicho, C. (2013b). Sample pretreatment strategies for total reflection X-ray fluorescence analysis: A tutorial review. Spectrochimica Acta Part B: Atomic Spectroscopy, 90, 23-54.

De la Calle, I., Costas, M., Cabaleiro, N., Lavilla, I. \& Bendicho, C. (2012). Use of high-intensity sonication for pre-treatment of biological tissues prior to multielemental analysis by total reflection X-ray fluorescence spectrometry. Spectrochimica Acta Part B: Atomic Spectroscopy, $67,43-49$.

De la Calle, I., Costas, M., Cabaleiro, N., Lavilla, I. \& Bendicho, C. (2013c). Fast method for multielemental analysis of plants and discrimination according to the anatomical part by total reflection X-ray fluorescence spectrometry. Food Chemistry 138, 234-241.

De la Calle, I., Pena, F., Cabaleiro, N., Lavilla, I. \& Bendicho, C. (2011b). Ion pair-based dispersive liquid-liquid microextraction for gold determination at ppb level in solid samples after 
ultrasound-assisted extraction and in waters by electrothermal-atomic absorption spectrometry. Talanta, 84, 109-115.

De la Guardia, M. \& Armenta, S. (2011). Avoiding Sample treatments. In D. Barceló (Ed.), Green Analytical Chemistry: Theory \& Practice (pp. 59-86). Oxford: Elsevier.

De Oliveira, E. (2003). Sample Preparation for Atomic Spectroscopy: Evolution and Future Trends, Journal of Brazilian Chemical Society, 14, 174-182.

De Paula, C. E. R., Caldas, L. F. S., Brum, D. M. \& Cassella, R. J. (2012). Development of an ultrasonic slurry sampling method for the determination of $\mathrm{Cu}$ and $\mathrm{Mn}$ in antibiotic tablets by electrothermal atomic absorption Spectrometry. Journal of Pharmaceutical and Biomedical Analysis, 66, 197- 203.

De Vevey, E., Bitton, G., Rossel, D., Ramos, L. D., Munguia-Gerrero, L. \& Tarradellas, J. (1993). Concentration and bioavailability of heavy metals in sediments in lake yojoa (honduras). Bulletin of Environmental Contamination and Toxicology, 50, 253-259.

Deaker, M. \& Maher, W. (1997). Low volume microwave digestion for the determination of selenium in marine biological tissues by graphite furnace atomic absorption spectroscopy. Analytica Chimica Acta, 350, 287-294.

Deaker, M. \& Maher, W. (1999). Determination of arsenic in arsenic compounds and marine biological tissues using low volume microwave digestion and electrothermal atomic absorption spectrometry. Journal of Anaytical Atomic Spectrometry, 14, 1193-1207.

Del Castilho, P. \& Breder, R. (1997). Soils and soil solutions. In M. Stoeppler (Ed.) Sampling and sample preparation (pp. 43-56). Germany: Springer-Verlag Berlin Heidelberg.

Delgado, A., Usobiaga, A., Prieto, A., Zuloaga, O., de-Diego, A. \& Madariaga, J. M., (2008). Optimisation of the headspace-solid phase microextraction for organomercury and organotin compound determination in sediment and biota. Journal of Separation Science, 31, 768-774.

Deng, G. \& Collins, G. E. (2003). Nonaqueous based microchip separation of toxic metal ions using 2-(5-bromo-2-pyridylazo)-5-(N-propyl- $N$-sulfopropylamino)phenol. Journal of Chromatography A, 989, 311-316.

Dobrowolski, R. \& Otto, M. (2012). Determination of nickel and cobalt in reference plant materials by carbon slurry sampling GFAAS technique after their simultaneous preconcentration onto modified activated carbon. Journal of Food Composition and Analysis, 26, 58-65.

Dobrowolski, R. \& Otto, M. (2013). Preparation and evaluation of Fe-loaded activated carbon for enrichment of selenium for analytical and environmental purposes. Chemosphere, 90 , 683-690.

Dobrowolski, R., Adamczyk, A. \& Otto, M. (2013). Determination of vanadium in soils and sediments by the slurry sampling graphite furnace atomic absorption spectrometry using permanent modifiers. Talanta, 113, 19-25.

Dobrowolski, R., Kurylo, M., Otto, M. \& Mróz, A. (2012). Determination of gold in geological materials by carbon slurry sampling graphite furnace atomic absorption spectrometry. Talanta, 99, 750-757.

Doménech-Carbó, A., Labuda, J., Scholz, F. (2013). Electroanalytical chemistry for the analysis of solids: Characterization and classification (IUPAC Technical Report). Pure and Applied Chemistry, 85, 609-631.

Domeño, C., Blasco, M., Sánchez, C. \& Nerín, C. (2006). A fast extraction technique for extracting polycyclic aromatic hydrocarbons (PAHs) from lichens samples used as biomonitors of air pollution: Dynamic sonication versus other methods. Analytica Chimica Acta, 569, 103-112.

Domeño, C., Munizza, G. \& Nerín, C. (2005). Development of a solid-phase microextraction method for direct determination of pentachlorophenol in paper and board samples: Comparison with conventional extraction method. Journal of Chromatography A, 1095, 8-15.

Domínguez-González, R., Moreda-Piñeiro, A., Bermejo-Barrera, A. \& Bermejo-Barrera, P. (2005). Application of ultrasound-assisted acid leaching procedures for major and trace elements 
determination in edible seaweed by inductively coupled plasma-optical emission spectrometry. Talanta, 66, 937-942.

Donard, O. F. X., Lalere, B., Martin, F. \& Lobinski, R., (1995). Microwave-assisted leaching of organotin compounds from sediments for speciation analysis. Analytical Chemistry, 67, 4250-4254.

Du, W., Lei, C., Zhang, S., Bai, G., Zhou, H., Sun, M., Fu, Q. \& Chang, C. (2014). Determination of clenbuterol from pork samples using surface molecularly imprinted polymers as the selective sorbents for microextraction in packed syringe. Journal of Pharmaceutical and Biomedical Analysis, 91, 160-168.

Duarte, F. A., Pereira, J. S. F., Barin, J. S., Mesko, M. F., Dressler, V. L., Flores, E. M. D. \& Knapp, G. (2009). Seafood digestion by microwave-induced combustion for total arsenic determination by atomic spectrometry techniques with hydride generation. Journal of Analytical Atomic Spectrometry, 24, 224-227.

Ducree, J., Haeberle, S., Lutz, S., Pausch, S., von Stetten, F. \& Zengerle, R. (2007). The centrifugal microfluidic bio-disk platform. Journal of Micromechanics and Microengineering, 17, S103S115.

Duford, D. A., Peng, D. D. \& Salin, E. D. (2009). Magnetically driven solid sample preparation for centrifugal microfluidic devices. Analytical Chemistry, 81, 4581-4584.

Ebdon, L., Fisher, A.S., Betti, M. \& Leroy, M. (2003). Detection methods for the quantitation of trace elements. In Z. Mester \& R. Sturgeon (Eds.), Sample preparation for trace element analysis (pp. 117-190). The Netherlands: Elsevier.

Eilola, K. \& Perämäki., P. (2001). Microwave heated vapor-phase digestion method for biological sample materials. Fresenius Journal Analytical Chemistry, 369, 107-112.

Eilola, K. \& Perämäki., P. (2003). Development of a modified medium pressure microwave vapor-phase digestion method for difficult to digest organic samples. The Analyst, 128, 194-197.

Eilola, K. \& Perämäki., P. (2009). Microwave-assisted double insert vapour-phase digestion of organic samples. Analytica Chimica Acta, 634, 205-208.

El Azouzi, H., Cervera, M. L. \& de la Guardia, M. (1998). Multi-elemental analysis of mussel samples by atomic absorption spectrometry after room temperature sonication. Journal of Analytical Atomic Spectrometry, 13, 533-538.

El-Amrani, S., Pena-Abaurrea, M., Sanz-Landaluze, J., Ramos, L., Guinea, J. \& Cámara, C. (2012). Bioconcentration of pesticides in Zebrafish eleutheroembryos (Daniorerio). Science of Total Environment, 425, 184-190.

Elik, A. (2005). Ultrasound assisted pseudo-digestion of street dust samples prior to determination by atomic absorption spectrometry. Talanta, $66,882-888$.

Esslemont, G., Maher, W., Ford, P. \& Krikowa, F. (2000). The determination of phosphorus and other elements in plant leaves by ICP-MS after low-volume microwave digestion with nitric acid. Atomic Spectroscopy, 21, 42-45.

Felton, M. J. (2003). Lab on a chip: poised on the brink. Analytical Chemistry, 75, 505A-508A.

Ferreira, S. L. C. , Miró, M., da Silva, E. G. P., Matos, G. D., dos Reis, P. S., Cardoso-Brandao, G. C., dos Santos, W. N. L., Tavares-Duarte, A. T., Rodrigues-Vale, M. G. R. \& Araujo, R. G. A. (2010). Slurry sampling-an analytical strategy for the determination of metals and metalloids by spectroanalytical techniques. Applied Spectroscopy Reviews, 45, 44-62.

Ferreira-Damin, I. C., Zmozinski, A. V., Borges, A. R., Vale, M. G. R. \& da Silva, M. M. (2011) Determination of cadmium and lead in fresh meat by slurry sampling graphite furnace atomic absorption spectrometry. Analytical Methods, 3, 1379-1385.

Figueredo-Rego, J., Virgilio, Nóbrega, J. A. \& Gomes-Neto, J. A. (2012). Determination of lead in medicinal plants by high-resolution continuum source graphite furnace atomic absorption spectrometry using direct solid sampling. Talanta, 100, 21-26. 
Filgueiras, A. V., Capelo, J. L., Lavilla, I. \& Bendicho, C. (2000). Comparison of ultrasound-assisted extraction and microwave-assisted digestion for determination of magnesium, manganese and zinc in plant samples by flame atomic absorption spectrometry. Talanta, 53, 433-441.

Filgueiras, A. V., Lavilla, I. \& Bendicho, C. (2001). Ultrasound-assisted solubilization of trace and minor metals from plant tissue using ethylenediaminetetraacetic acid in alkaline medium. Fresenius Journal Analytical Chemistry, 369, 451-456.

Filgueiras, A. V., Lavilla, I. \& Bendicho, C. (2002a). Chemical sequential extraction for metal partitioning in environmental solid samples. Journal of Environmental Monitoring, 4, 823-857.

Filgueiras, A. V., Lavilla, I. \& Bendicho, C. (2002b). Comparison of the standard SM\&T sequential extraction method with small-scale ultrasound-assisted single extractions for metal partitioning in sediments. Analytical and Bioanalytical Chemistry, 374, 103-108.

Flores, E. M. M., Barin, J. S., Mesko, M. F. \& Knapp, G. (2007). Sample preparation techniques based on combustion reactions in closed vessels $-A$ brief overview and recent applications. Spectrochimica Acta Part B: Atomic Spectroscopy, 62, 1051-1064.

Flores, E.M. (1997); Ph.D Thesis, Universidade Federal do Rio Grande do Sul, Porto Alegre.

Gallagher, P. A., Shoemaker, J. A., Wei, X., Brockhoff-Schwegel, C. A. \& Creed, J. T. (2001). Extraction and detection of arsenicals in seaweed via accelerated solvent extraction with ion chromatographic separation and ICP-MS detection. Fresenius Journal Analytical Chemistry, 369, 71-80.

Garrigues, S. \& de la Guardia, M. (2013). Non-invasive analysis of solid samples. TrAC Trends in Analytical Chemistry, 43, 161-173.

Gil, S., Fragueiro, S., Lavilla, I. \& Bendicho, C. (2005). Determination of methylmercury by electrothermal atomic absorption with in situ hydride generation. Spectrochimica Acta Part B: Atomic Spectroscopy, 60, 145-150.

Gleit, C. E. \& Holland, W. D. (1962). Use of electrically excited oxygen for the low temperature decomposition of organic substances. Analytical Chemistry, 34, 1454-1457.

Goenaga-Infante, H., O’Connor, G., Rayman, M., Wahlen, R., Entwisle, J., Norris, P., Hearn, R. \& Catterick, T. (2004). Selenium speciation analysis of selenium-enriched supplements by HPLC with ultrasonic nebulisation ICP-MS and electrospray MS/MS detection. Journal of Analytical Atomic Spectrometry, 19, 1529-1538.

Goenaga-Infante, H., O’Connor, G., Rayman, M., Wahlen, R., Spallholz, J. E., Hearn, R. \& Catterick, T. (2005). Identification of water-soluble gamma-glutamyl-Se-methylselenocysteine in yeast-based selenium supplements by reversed-phase HPLC with ICP-MS and electrospray tandem MS detection. Journal of Analytical Atomic Spectrometry, 20, 864-870.

González-Curbelo, M. A., Hernández-Borges, J., Borges-Miquel, T. M. \& Rodríguez-Delgado, M. A. (2013). Determination of organophosphorus pesticides and metabolites in cereal-based baby foods and wheat flour by means of ultrasound-assisted extraction and hollow-fiber liquid-phase microextraction prior to gas chromatography with nitrogen phosphorus detection. Journal of Chromatography A, 1313, 166-174.

Greenway, G. M. \& Song, Q. J. (2002). An ultrasound accelerated sequential extraction method and its application for element partitioning studies in compost from mixed waste streams. Journal of Environmental Monitoring, 4, 950-955.

Grinberg, P., Campos, R. C., Mester, Z. \& Sturgeon, R. E. (2003). A comparison of alkyl derivatization methods for speciation of mercury based on solid phase microextraction gas chromatography with furnace atomization plasma emission spectrometry detection. Journal of Analytical Atomic Spectrometry, 18, 902-909.

Guan, H., Brewer, W. E., Garris, S. T. \& Morgan. S. L. (2010). Disposable pipette extraction for the analysis of pesticides in fruit and vegetables using gas chromatography/mass spectrometry. Journal of Chromatography A, 1217, 1867-1874.

Günther, D. \& Hattendorf, B. (2005). Solid sample analysis using laser ablation inductively coupled plasma mass spectrometry. TrAC Trends in Analytical Chemistry, 24, 255- 265. 
Guo, L. \& Kee Lee, H. (2013). Microwave assisted extraction combined with solvent bar microextraction for one-step solvent-minimized extraction, cleanup and preconcentration of polycyclic aromatic hydrocarbons in soil samples. Journal of Chromatography A, 1286, 9-15,

Gutiérrez Valencia, T. M. \& García de Llasera, M. P. (2011). Determination of organophosphorus pesticides in bovine tissue by an on-line coupled matrix solid-phase dispersion-solid phase extraction-high performance liquid chromatography with diode array detection method. Journal of Chromatography A, 1218, 6869- 6877.

Halls, D. J. (1995). Analytical minimalism applied to the determination of trace elements by atomic spectrometry. Journal of Analytical Atomic Spectrometry, 10, 169-175.

Hansson, E. \& Hakkarainen, M. (2006). Multiple headspace single-drop microextraction-a new technique for quantitative determination of styrene in polystyrene. Journal of Chromatography A, 1102, 91-95.

Hawthorne, S. B., Trembley, S., Moniot, C. L., Grabanski, C. B. \& Miller, D. J. (2000). Static subcritical water extraction with simultaneous solid-phase extraction for determining polycyclic aromatic hydrocarbons on environmental solids. Journal of Chromatography A, 886, 237-244.

He, D., Zhang, Z., Huang, Y. \& Hu, Y. (2007). Chemiluminescence microflow injection analysis system on a chip for the determination of nitrite in food. Food Chemistry, 10, 667-672.

Herrero-Latorre, C., Alvarez-Mendez, J., Barciela-Garcia, J., Garcia-Martin, S. \& Peña-Crecente, R. M. (2012). Carbon nanotubes as solid-phase extraction sorbents prior to atomic spectrometric determination of metal species: A review. Analytica Chimica Acta, 749, 16- 35.

Hirata, S. \& Toshimitsu, H. (2005). Determination of arsenic species and arsenosugars in marine samples by HPLC-ICP-MS. Analytical and Bioanalytical Chemistry, 383, 454-460.

Hodson, M. J., White, P.J., Mead, A. \& Broadley, M. R. (2005). Phylogenetic variation in the silicon composition of plants. Annals of Botany, 96, 1027-1046.

Hoenig, M. \& de Kersabiec, A.-M. (1996). Review Sample preparation steps for analysis by atomic spectroscopy methods: present status. Spectrochimica Acta Part B: Atomic Spectroscopy, 51, 1297-1307.

Hoenig, M. (2001). Preparation steps in environmental trace element analysis-facts and traps. Talanta, 54, 1021-1038.

Hoenig, M. (2003). Dry ashing. In Z. Mester \& R. Sturgeon (Eds.), Sample preparation of geological samples, soils and sediment (pp. 235-255). The Netherlands: Elsevier.

Hoenig, M., Baeten, H., Vanhentenrijk, S., Vassileva, E. \& Quevauviller, P. (1998). Critical discussion on the need for an efficient mineralization procedure for the analysis of plant material by atomic spectrometric methods. Analytica Chimica Acta, 358, 85-94.

Hong, Y. \& Chen, L. (2013). Extraction of quercetin from Herba Lysimachiae by molecularly imprintedmatrix solid phase dispersion, Journal of Chromatography B: Analytical Technologies in the Biomedical and Life Sciences, 941, 38-44.

Horváth, M., Halász, G., Kucanová, E., Kuciková, B., Fekete, I., Remeteiová, D., Heltai, G. \& Flórián, K. (2013). Sequential extraction studies on aquatic sediment and biofilm samples for the assessment of heavy metal mobility. Microchemical Journal, 107, 121-125.

Hristozov, D., Domini, C. E., Kmetov, V., Stefanova, V., Georgieva, D. \& Canals, A. (2004). Direct ultrasound-assisted extraction of heavy metals from sewage sludge samples for ICP-OES analysis. Analytica Chimica Acta, 516, 187-196.

Hsu, W.-H., Jiang, S.-J., Sahayam A. C. (2013). Determination of Pd, Rh, Pt, Au in road dust by electrothermal vaporization inductively coupled plasma mass spectrometry with slurry sampling. Analytica Chimica Acta, 794, 15- 19.

Huang, J., Liu, J., Zhang, C., Wei, J., Mei, L., Yu, S., Li, G. \& Xu, L. (2012). Determination of sulfonamides in food samples by membrane-protected micro-solid phase extraction coupled with high performance liquid chromatography. Journal of Chromatography A, 1219, 66-74. 
Huang, M., Chen, H., Fu, S. \& Ding, W. (2013). Determination of volatile N-nitrosamines in meat products by microwave-assisted extraction coupled with dispersive micro solid-phase extraction and gas chromatography -chemical ionisation mass spectrometry. Food Chemistry, 138, 227-233.

Huerga, A., Lavilla, I. \& Bendicho, C. (2005). Speciation of the immediately mobilisable As(III), $\mathrm{As}(\mathrm{V}), \mathrm{MMA}$ and DMA in river sediments by high performance liquid chromatography-hydride generation-atomic fluorescence spectrometry following ultrasonic extraction. Analytica Chimica Acta, 534, 121-128.

Hwang, S.-S., Park, J.-S. \& Namkoong, W. (2007). Ultrasonic-assisted extraction to release heavy metals from contaminated soil. Journal of Industrial and Engineering Chemistry, 13, 650-656.

Hyötyläinen, T. (2009). Critical evaluation of sample pretreatment techniques. Analytical and Bioanalytical Chemistry, 394, 743-758.

Hyötyläinen, T., Andersson, T, Hartonen, K., Kuosmanen, K. \& Riekkola, M. L. (2000). Pressurized hot water extraction coupled on-line with LC-GC: Determination of polyaromatic hydrocarbons in sediment. Analytical Chemistry, 72, 3070-3076.

Iavicoli, I., Castellino, N., Carelli, G. \& Schlemmer, G. (2001). Lead determination in ultra micro samples of animal diet using graphite furnace atomic absorption spectrometry. Atomic Spectroscopy, 22, 319-323.

Ibarlucea, B., Díez-Gil, C., Ratera, I., Veciana, J., Caballero, A., Zapata, F., Tárraga, A., Molina, P., Demming, S., Büttgenbach, S., Fernández-Sánchez, C. \& Llobera, A. (2013). PDMS based photonic lab-on-a-chip for the selective optical detection of heavy metal ions. The Analyst, 138, 839-844.

leggli, C. V. S., Bohrer D., do Nascimento, P. C., de Carvalho, L. M. \& Garcia, S. C. (2010). Determination of sodium, potassium, calcium, magnesium, zinc, and iron in emulsified egg samples by flame atomic absorption spectrometry. Talanta, 80, 1282-1286.

Ihnat M. (2003). Sample preparation for food analysis In Z. Mester \& R. Sturgeon (Eds.), Sample preparation for trace element analysis (pp. 765-855). The Netherlands: Elsevier.

Ilander, A. \& Väisänen, A. (2007). An ultrasound-assisted digestion method for the determination of toxic element concentrations in ash samples by inductively coupled plasma optical emission spectrometry. Analytica Chimica Acta, 602, 195-201.

Ilander, A. \& Väisänen, A. (2009). The determination of trace element concentrations in fly ash samples using ultrasound-assisted digestion followed with inductively coupled plasma optical emission spectrometry. Ultrasonics Sonochemistry, 16, 763-768.

Intima, D. P., de Oliveira, E. \& Oliveira, P. V. (2009). Feasibility of using in situ fusion for the determination of $\mathrm{Co}, \mathrm{Cr}$ and $\mathrm{Mn}$ in Portland cement by direct solid sampling graphite furnace atomic absorption spectrometry. Spectrochimica Acta Part B: Atomic Spectroscopy, 64, 610-614.

Ipolyi, I., Brunori, C., Cremisini, C., Fodor, P., Macaluso, L. \& Morabito, R. (2002). Evaluation of performance of time-saving extraction devices in the BCR three-step sequential extraction procedure. Journal of Environmental Monitoring, 4, 541-548.

Jamali, M. K., Kazi, T. G., Arain, M. B., Afridi, H. I., Jalbani, N., Kandhro, G. A., Shah, A. Q. \& Baig, J.A. (2009). Speciation of heavy metals in untreated sewage sludge by using microwave assisted sequential extraction procedure. Journal of Hazardous Materials, 163, 1157-1164.

Jiao, J., Ma, D., Gai, Q., Wang, W., Luo, M., Fu, Y. \& Ma, W. (2013). Rapid analysis of Fructusforsythiae essential oil by ionic liquids-assisted microwave distillation coupled with headspace single-drop microextraction followed by gas chromatography-mass spectrometry. Analytica Chimica Acta, 804, 143-150.

Kadenkin, A., Amberger, M., Fittschen, U. E. A. \& Broekaert, J. A. C. (2011). Micro-homogeneity studies of boron carbide powders. Microchimica Acta, 173, 183-188. 
Karageorgou, E. \& Samanidou, V. (2010). Application of ultrasound-assisted matrix solid-phase dispersion extraction to the HPLC confirmatory determination of cephalosporin residues in milk. Journal of Separation Science, 33, 2862-2871.

Karageorgou, E. \& Samanidou, V. (2011). Development and validation according to European Union Decision 2002/657/EC of an HPLC-DAD method for milk multiresidue analysis of penicillins and amphenicols based on dispersive extraction by QuEChERS in MSPD format. Journal of Separation Science, 34, 1893-1901.

Karageorgou, E., Armeni, M., Moschou, I. \& Samanidou, V. (2014). Ultrasound-assisted dispersive extraction for the high pressure liquid chromatographic determination of tetracyclines residues in milk with diode array detection, Food Chemistry, 150, 328-334.

Karageorgou, E., Myridakis, A., Stephanou, E. \& Samanidou, V. (2013). Multi-residue LC-MS/MS analysis of cephalosporins and quinolones in milk following ultrasound assisted matrix solid phase dispersive extraction combined with QuEChERS methodology. Journal of Separation Science, 36, 2020-2027.

Karageorgou, E., Samanidou, V. \& Papadoyannis, I. (2012). Ultrasound-assisted matrix solid phase dispersive extraction for the simultaneous analysis of blactams (four penicillins and eight cephalosporins) in milk by high performance liquid chromatography with photodiode array detection. Journal of Separation Science, 35, 2599-2607.

Kazi, T. G., Jalbani, N., Arain, M. B., Jamali, M. K., Afridi, H. I. \& Shah, A. Q. (2009a). Determination of toxic elements in different brands of cigarette by atomic absorption spectrometry using ultrasonic assisted acid digestion. Environmental Monitoring and Assessment, 154, 155-167.

Kazi, T. G., Jalbani, N., Baig, J. A., Arain, M. B., Afridi, H. I., Jamali, M. K., Shah A. Q. \& Memon, A. N. (2010). Evaluation of toxic elements in baby foods commercially available in Pakistan. Food Chemistry, 119, 1313-1317.

Kazi, T. G., Jamali, M. K., Arain, M. B., Afridi, H. I., Jalbani, N., Sarfraz, R. A. \& Ansari, R. (2009b). Evaluation of an ultrasonic acid digestion procedure for total heavy metals determination in environmental and biological samples. Journal of Hazardous Materials, 161, 1391-1398.

Kazi, T. G., Jamali, M. K., Siddiqui, A., Kazi, G. H., Arain, M. B. \& Afridi, H. I. (2006). An ultrasonic assisted extraction method to release heavy metals from untreated sewage sludge samples. Chemosphere, 63, 411-420.

Ke, Y., Zhua, F., Jiang, R., Wang, Y., Yue, L., Liu, H., Zeng, F. \& Ouyang, G. (2014). Determination of polycyclic aromatic hydrocarbons in leather products using solid-phase microextraction coupled with gas chromatography-mass spectrometry. Microchemical Journal, 112, 159-163.

Khuder, A., Sawan, M. K., Karjou, J., \& Razouk, A. K. (2009). Determination of trace elements in Syrian medicinal plants and their infusions by energy dispersive $X$-ray fluorescence and total reflection X-ray fluorescence spectrometry. Spectrochimica Acta Part B: Atomic Spectroscopy, 64, 721-725.

Kim, N., Jung, M., Yoo, Z., Lee, S. \& Lee, D. (2005). Headspace hanging drop liquid phase microextraction and GC-MS for the determination of linalool from evening primrose flowers. Bulletin of the Korean Chemistry Society, 26, 1996-2000.

Klitenick, M. A., Frederickson, C. J. \& Manton, W. I. (1983). Acid-vapor decomposition for determination of zinc in brain tissue by isotope dilution mass spectrometry. Analytical Chemistry, 55, 921-923.

Kokosa, J., Przyjazny, A. \& Jeannot, M. A. (2009). Solvent microextraction; Theory and practice, New Jersey: John Wiley \& Sons Inc.

Kolmogorov, Y. P., Mezentsev, N. A., Mironov, A. G., Parkhomenko,V. S., Spiridonov, A. M., Shaporenko, A. D., Yusupov, T. S., Zhmodik, S. M., Zolotarev, K. V. \& Anoshin, G. N. (2009). Development of a complex of instrumental nuclear-physical methods to detect PGE, Re, Au, and Ag in hard-to-analyze rocks and complex ores. Nuclear Instruments and Methods in Physics Research Section A, 603, 131-133. 
Krasnodebska-Ostrega, B., Kaczorowska, M. \& Golimowski, J. (2006). Ultrasound-assisted extraction for the evaluation of element mobility in bottom sediment collected at mining and smelting $\mathrm{Pb}-$ Zn ores area in Poland. Microchimica Acta, 154, 39-43.

Kristenson, E. M., Haverkate, E. G. J, Slooten, C. J., Ramos, L., Vreuls, R. J. J. \& Brinkman, U. A. Th, (2001). Miniaturized automated matrix solid-phase dispersion extraction of pesticides in fruit followed by gas chromatographic-mass spectrometric analysis. Journal of Chromatography A, 917, 277-286.

Kristenson, E. M., Ramos, L. \& Brinkman, U. A. T. (2006). Recent advances in matrix solid-phase dispersion. TrAC Trends in Analytical Chemistry, 25, 96-111.

Kulkarni, P., Chellam, S. \& Mittlefehldt, D. W. (2007). Microwave-assisted extraction of rare earth elements from petroleum refining catalysts and ambient fine aerosols prior to inductively coupled plasma-mass spectrometry. Analytica Chimica Acta, 581, 247-259.

Lana, N. B., Berton, P., Covaci, A., Atencio, A. G., Ciocco, N. F. \& Altamirano, J. C. (2013). Ultrasound leaching-dispersive liquid-liquid microextraction based on solidification of floating organic droplet for determination of polybrominateddiphenyl ethers in sediment samples by gas chromatography-tandem mass spectrometry. Journal of Chromatography A, 1285, 15-21.

Landajo, A., Arana, G., de Diego, A., Etxebarria, N., Zuloaga, O. \& Amouroux, D. (2004). Analysis of heavy metal distribution in superficial estuarine sediments (estuary of Bilbao, Basque Country) by open-focused microwave-assisted extraction and ICP-OES. Chemosphere, 56, 1033-1041.

Lásztity, A., Krushevska, A., Kotrebai, M., Barnes, R. M. \& Amarasiriwardena, D. (1995). Arsenic determination in environmental, biological and food samples by inductively coupled plasma mass spectrometry. Journal of Analytical Atomic Spectrometry, 10, 505-510.

Lavilla, I., Cabaleiro, N., Costas, M., de la Calle, I. \& Bendicho C. (2009). Ultrasound-assisted emulsification of cosmetic samples prior to elemental analysis by different atomic spectrometric techniques. Talanta, 80, 109-116.

Lavilla, I., Cabaleiro, N., Pena, F., De La Calle, I. \& Bendicho, C. (2010). Ultrasound-assisted emulsification microextraction with simultaneous derivatization coupled to fibre optics-based cuvetteless UV-vis micro-spectrophotometry for formaldehyde determination in cosmetic samples. Analytica Chimica Acta, 674, 59-63.

Lavilla, I., Costas, M., Gil, S., Corderi, S., Sanchez, G. \& Bendicho, C. (2012). Simplified and miniaturized procedure based on ultrasound-assisted cytosol preparation for the determination of $\mathrm{Cd}$ and $\mathrm{Cu}$ bound to metallothioneins in mussel tissue by ICP-MS. Talanta, 93, 111-116.

Lavilla, I., Mosquera, A., Millos, J., Cameselle, J. \& Bendicho, C. (2006a). Ultrasound-assisted extraction technique for establishing selenium contents in breast cancer biopsies by Zeemanelectrothermal atomic absorption spectrometry using multi-injection. Analytica Chimica Acta, 566, 29-36.

Lavilla, I., Vilas, P. \& Bendicho, C. (2008). Fast determination of arsenic, selenium, nickel and vanadium in fish and shellfish by electrothermal atomic absorption spectrometry following ultrasound-assisted extraction. Food Chemistry, 106, 403-409.

Lavilla, I., Vilas, P., Millos, J. \& Bendicho, C. (2006b). Development of an ultrasound-assisted extraction method for biomonitoring of vanadium and nickel in the coastal environment under the influence of the Prestige fuel spill (North east Atlantic ocean), Analytica Chimica Acta, 577, 119-125.

Lee, K. S., Shiddiky, M. J. A., Park, S. H., Park, D. S. \& Shim, Y. B. (2008). Electrophoretic analysis of food dyes using a miniaturized microfluidic system. Electrophoresis, 29, 1910-1917.

Lee, T. P., Saad, B., Poh Ng, E. \& Salleh, B. (2012). Zeolite Linde Type L as micro-solid phase extraction sorbent for the high performance liquid chromatography determination of ochratoxin A in coffee and cereal. Journal of Chromatography A, 1237, 46-54.

Lehmann, E. L., Fostier, A. H. \& Arruda, M. A. Z. (2013). Hydride generation using a metallic atomizer after microwave-assisted extraction for inorganic arsenic speciation in biological samples. Talanta, 104, 187-192. 
Lesniewicz, A. \& Zyrnicki, W. (2003). Comparison of Conventional, Ultrasound- and microwaveassisted extraction techniques applied for the determination of metals in spruce needles. International Journal of Environmental Analytical Chemistry, 83, 735-748.

Ligor, T. \& Buszewski, B. (2008). Single-drop microextraction and gas chromatography-mass spectrometry for the determination of volatile aldehydes in fresh cucumbers. Analytical and Bioanalytical Chemistry, 391, 2283-2289.

Lima, E. C., Barbosa Jr., F., Krug, F. J., Silva M. M. \& Vale, M. G. R. (2000). Comparison of ultrasoundassisted extraction, slurry sampling and microwave-assisted digestion for cadmium, copper and lead determination in biological and sediment samples by electrothermal atomic absorption spectrometry. Journal of Analytical Atomic Spectrometry, 15, 995-1000.

Lin, F., Liu, D., Das, S. M., Prempeh, N., Hua, Y. \& Lu, J. (2014). Recent progress in heavy metal extraction by supercritical $\mathrm{CO}_{2}$ fluids. Industrial \& Engineering Chemistry Research, 53, 1866-1877.

Lin, M.-L. \& Jiang, S.-H. (2013). Determination of As, Cd, Hg and Pb in herbs using slurry sampling electrothermal vaporisation inductively coupled plasma mass spectrometry. Food Chemistry, 141, 2158-2162.

Liu, Q., Shi, J., Sun, J., Wang, T., Zeng, L., Zhu, N. \& Jiang, G. (2011). Graphene-assisted matrix solid-phase dispersion for extraction of polybrominated diphenyl ethers and their methoxylated and hydroxylatedanalogs from environmental samples. Analytica Chimica Acta, $708,61--68$.

Liu, W., Hwang, B., Li, Z., Jen, J. \& Lee, M. (2011). Headspace solid phase microextraction in-situ supercritical fluid extraction coupled to gas chromatography-tandem mass spectrometry for simultaneous determination of perfluorocarboxylic acids in sediments. Journal of Chromatography A, 1218, 7857-7863.

Liu, Y., Li, Y. \& Yang, X.-P. (2008) Preparation, characterization, and application of L-cysteine functionalized multiwalled carbon nanotubes as a selective sorbent for separation and preconcentration of heavy metals. Advanced Functional Materials, 18, 1536-1543.

Liu, Y., Li, Y., Wu, Z.-Q. \& Yan, X.-P. (2009). Fabrication and characterization of hexahistidine-tagged protein functionalized multi-walled carbon nanotubes for selective solid-phase extraction of $\mathrm{Cu}^{2+}$ and $\mathrm{Ni}^{2+}$. Talanta, 79, 1464-1471.

Liva, M., Muñoz-Olivas, R. \& Cámara C. (2000). Determination of Cd in sonicate slurries and leachates of biological and environmental materials by FI-CV-AAS. Talanta, 51, 381-387.

Long, X., Miró, M. \& Hansen, E. H. (2005). Universal approach for selective trace metal determinations via sequential injection-bead injection-lab-on-valve sing renewable hydrophobic bead surfaces as reagent carriers. Analytical Chemistry, 77, 6032-6040.

López-García, I., Campillo, N., Arnau-Jerez, I. \& Hernández-Córdoba, M. (2005). Electrothermal atomic absorption spectrometric determination of germanium in soils using ultrasoundassisted leaching. Analytica Chimica Acta, 531, 125-129.

Lu, Y. \& Zhu, Y. (2013). Determination of chlorobenzenes in textiles by pressurized hot water extraction followed by vortex-assisted liquid-liquid microextraction and gas chromatographymass spectrometry. Journal of Chromatography A, 1319, 27-34.

Lu, Y. \& Zhu, Y. (2014). Combination of accelerated solvent extraction and vortex-assisted liquidliquid microextraction for the determination of dimethyl fumarate in textiles and leathers by gas chromatography-mass spectrometry. Talanta, 119, 430-434.

Lu, Y., Shen, Q., Dai, Z., Zhang, H. \& Wang, J. (2011). Development of an on-line matrix solid-phase dispersion/fast liquid chromatography/tandem mass spectrometry system for the rapid and simultaneous determination of 13 sulfonamides in grass carp tissues. Journal of Chromatography A, 1218, 929-937.

Lukkari, T., Taavitsainen, M., Vaäisänen, A. \& Haimi, J. (2004). Effects of heavy metals on earthworms along contamination gradients in organic rich soils. Ecotoxicology and Environmental Safety, $59,340-348$. 
Luque de Castro, M. D. \& Luque-García, J. L. (2002). Acceleration and automation of solid sample pretreatment. Amsterdam: Elsevier.

Luque de Castro, M. D. \& Priego-Capote, F. (2007). Ultrasound-assisted preparation of liquid samples. Talanta, 72, 321-334.

Luque-García, J. L. \& Luque de Castro, M. D. (2003). Where is microwave-based analytical equipement for solid sample pre-treatment going? TrAC Trends in Analytical Chemistry, 22, 90-98.

MacDonald, A. M. G. (1961). The oxygen flask method. The Analyst, 86, 3-12.

Maduro, C., Vale, G., Alves, S., Galesio, M., Gomes da Silva, M. D. R., Fernandez, C., Catarino, S., Rivas, M. G., Mota, A. M. \& Capelo, J. L. (2006). Determination of Cd and Pb in biological reference materials by electrothermal atomic absorption spectrometry: A comparison of three ultrasonic-based sample treatment procedures. Talanta, 68, 1156-1161.

Magalhães, T., Carvalho, M. L., von Bohlen, A. \& Becker, M. (2010). Study on trace elements behaviour in cancerous and healthy tissues of colon, breast and stomach: total reflection X-ray fluorescence applications, Spectrochimica Acta Part B: Atomic Spectroscopy, 65, 493-498.

Mages, M., Bandow, N., Küster, E., Brack, W. \& von Tümpling, W. (2008). Zinc and cadmium accumulation in single zebrafish (Danio rerio) embryos - a total reflection X-ray fluorescence spectrometry application. Spectrochimica Acta Part B: Atomic Spectroscopy, 63, 1443-1449.

Mages, M., Woelfl, S., Ovari, M., von Tümpling Jr., W. \& Encina, F. (2004.) The use of a portable total reflection X-ray fluorescence spectrometer for trace element determination in freshwater microcrustaceans (Daphnia). Spectrochimica Acta Part B: Atomic Spectroscopy, 59, 1265-1272.

Maher, W., Forster, S., Krikowa, F., Snitch, P., Chapple, G. \& Craig, P. (2001). Measurement of trace elements and phosphorus in marine animal and plant tissues by low-volume microwave digestion and ICP-MS. Atomic Spectroscopy, 22, 361-370.

Marcó, L. M., Capote, T., Hernández, E. A. \& Greaves, E. D. (2001). Feasibility study on in situ microwave digestion prior to analysis of biological samples by total reflection X-ray fluorescence. Spectrochimica Acta Part B: Atomic Spectroscopy, 56, 2187-2193.

Marín, A., López-Gonzálvez, A. \& Barbas, C. (2001). Development and validation of extraction methods for determination of zinc and arsenic speciation in soils using focused ultrasound: Application to heavy metal study in mud and soils. Analytica Chimica Acta, 442, 305-318.

Mark, D., Haeberle, S., Roth, G., von Stetten, F. \& Zengerle, R. (2010). Microfluidic lab-on-a-chip platforms: requirements, characteristics and applications. Chemical Society Reviews, 39, 1153-1182.

Mark, J. J. P., Kumar, A., Demattio, H., Hoffmann, W., Malik, A. \& Matysik, F. M. (2011). Combination of headspace single-drop microextraction, microchip electrophoresis and contactless conductivity detection for the determination of aliphatic amines in the biodegradation process of seafood samples. Electroanalysis, 23, 61-168.

Márquez-Sillero, I., Aguilera-Herrador, E., Cárdenas, S. \& Válcarcel, M. (2011). Ion-mobility spectrometry for environmental analysis. TrAC Trends in Analytical Chemistry, 30, 677-690.

Martínez-Fernández, M., Barciela-Alonso, M. C., Moreda-Piñeiro, A. \& Bermejo-Barrera, P. (2011). Matrix solid phase dispersion-assisted BCR sequential extraction method for metal partitioning in surface estuarine sediments. Talanta, 83, 840-849.

Mason, T. \& Lorimer, J. (2002). Applied Sonochemistry. The Uses of Power Ultrasound in Chemistry and Processing. Germany: Wiley-VCH.

Matin, A. A., Biparva, P., Gheshlaghi, M., Farhadi, K. \& Gheshlaghi, A. (2013). Environmental monitoring of complex hydrocarbon mixtures in water and soil samples after solid phase microextraction using PVC/MWCNTs nanocomposite fiber. Chemosphere, 93, 1920-1926.

Matos, W. O., Menezes, E. A., Gonzalez, M. H., Costa, L. M., Trevizan, L. C. \& Nogueira, A. R. A. (2009). Partial microwave-assisted wet digestion of animal tissue using a baby-bottle sterilizer 
for analyte determination by inductively coupled plasma optical emission spectrometry. Spectrochimica Acta Part B: Atomic Spectroscopy, 64, 615-618.

Matschat, R., Haßler, J., Traub, H. \& Dette, A. (2005). Multielement trace determination in SiC powders: assessment of interlaboratory comparisons aimed at the validation and standardization of analytical procedures with direct solid sampling based on ETV ICP OES and DC arC OES. Analytical and Bioanalytical Chemistry, 383, 1060-1074.

Matsubara, A., Harada, K., Hirata, K., Fukusaki, E. \& Bamba, T. (2012). High-accuracy analysis system for the redox status of coenzyme Q10 by online supercritical fluid extraction-supercritical fluid chromatography/mass spectrometry. Journal of Chromatography A, 1250, 7679.

Matusiewicz, H. \& Golik, B. (2004). Determination of major and trace elements in biological materials by microwave induced plasma optical emission spectrometry (MIP-OES) following tetramethylammonium hydroxide (TMAH) solubilisation. Microchemical Journal, 76, 23-29.

Matusiewicz, H. (1989). Acid vapour-phase pressure decomposition for the determination of elements in biological materials by flame atomic emission, spectrometry. Journal of Analytical Atomic Spectrometry. 4, 265-269.

Matusiewicz, H. (1991). A review of acid vapor-phase sample digestion of inorganic and organic matrices for elemental analysis, International Journal of Spectroscopy, 3, 22-28.

Matusiewicz, H. (2003). Wet digestion methods. In Z. Mester, R. Sturgeon (Eds.) Sample preparation for trace element analysis (pp. 193-233), Amsterdam, Elsevier.

Matusiewicz, H., Sturgeon, R. E. \& Bernan, S. S. (1991). Vapor-phase acid digestion of inorganic and organic matrixes for trace element analysis using a microwave heated bomb. Journal of Analytical Atomic Spectrometry, 6, 283-287.

McKiernan, J. W., Creed, J. T., Brockhoff, C. A., Caruso, J. A. \& Lorenzana, R. M. (1999). A comparison of automated and traditional methods for the extraction of arsenicals from fish. Journal of Analytical Atomic Spectrometry, 14, 607-613.

Mehdinia, A. \& Aziz-Zanjani, M. O. (2013). Advances for sensitive, rapid and selective extraction in different configurations of solid-phase microextraction. TrAC Trends in Analytical Chemistry, 51, 13-22.

Meloche, L. M., de Bruyn, A. M. H., Otton, S. V., Ikonomou, M. G. \& Gobas, F. A. P. C. (2009). Assessing exposure of sediment biota to organic contaminants by thin-film solid phase extraction. Environmental Toxicology andl Chemistry, 28, 247-253.

Mesko, M. F., Hartwig, C. A., Bizzi, C.A., Pereira, J.S.F., Mello, P.A. \& Flores, E. M. M. (2011). Sample preparation strategies for bioinorganic analysis by inductively coupled plasma mass spectrometry, International Journal of Mass Spectrometry, 307, 123-136.

Mesko, M. F., Mello, P. A., Bizzi, C. A., Dressler, V. L., Knapp, G \& Flores, E. M. M. (2010). Iodine determination in food by inductively coupled plasma mass spectrometry after digestion by microwave-induced combustion. Analytical and Bioanalytical Chemistry, 398, 1125-1131.

Mester, Z. \& Sturgeon, R. E. (2002). Detection of volatile organometal chloride species in model atmosphere above seawater and sediment. Environment Science and Technology, 36, $1198-1201$.

Mester, Z. (2002). Gas phase sampling of volatile (organo)metallic compounds above solid samples. Journal of Analytical Atomic Spectrometry, 17, 868-871.

Mester, Z., Lam, J., Sturgeon, R. \& Pawliszyn, J. (2000). Determination of methylmercury by solid-phase microextraction inductively coupled plasma mass spectrometry: a new sample introduction method for volatile metal species. Journal of Analytical Atomic Spectrometry, 15, 837-842.

Mierzwa, J., Adeloju, S. B. \& Dhindsa, H. S. (1997). Ultrasound accelerated solid-liquid extraction for the determination of selenium in biological samples by electrothermal atomization atomic absorption spectrometry. Analytical Sciences, 13, 189-193. 
Miller-Ihli, N. J. (1989). Slurry sample preparation for simultaneous multi-element graphite furnace atomic absorption spectrometry, Journal of Analytical Atomic Spectrometry, 3, 73-81.

Miller-Ihli, N. J. (1993). Advances in ultrasonic slurry graphite furnace atomic absorption spectrometry. Fresenius Journal Analytical Chemistry, 345, 482-489.

Millos, J., Costas-Rodríguez, M., Lavilla, I. \& Bendicho, C. (2009). Multiple small volume microwaveassisted digestions using conventional equipment for multielemental analysis of human breast biopsies by inductively coupled plasma optical emission spectrometry. Talanta, 77, 1490-1496.

Millos, J., Costas-Rodríguez, M., Lavilla, I. \& Bendicho, C. (2008). Multielemental determination in breast cancerous and non-cancerous biopsies by inductively coupled plasma-mass spectrometry following small volume microwave-assisted digestion. Analytica Chimica Acta, $622,77-84$.

Miró, M. \& Hansen, E. H. (2007). Miniaturization of environmental chemical assays in flowing systems: The lab-on-a-valve approach vis-à-vis lab-on-a-chip microfluidic devices. Analytica Chimica Acta, 600, 46-57.

Miró, M., Hansen, E. H. \& Buanuam, J. (2006). The potentials of the third generation of flow injection analysis for nutrient monitoring and fractionation analysis. Environmental Chemistry, 3, 26-30.

Mishra, S., Tripathi, R. M., Bhalke, S., Shukla, V. K. \& Puranik, V. D. (2005). Determination of methylmercury and mercury (II) in a marine ecosystem using solid-phase microextraction gas chromatography-mass spectrometry. Analytica Chimica Acta, 551, 192-198.

Mitra, S. \& Brukh, R. (2003). Sample preparation: an analytical perspective. In S. Mitra (Ed.) Sample preparation techniques in analytical chemistry (pp.1-36), Hoboken, N.J.: John Wiley \& Sons, Inc.

Moliner-Martinez, Y., Campíns-Falcó, P., Molins-Legua, C., Segovia-Martínez, L. \& Seco-Torrecillas, A. (2009). Miniaturized matrix solid phase dispersion procedure and solid phase microextraction for the analysis of organochlorinated pesticides and polybrominated diphenylethers in biota samples by gas chromatography electron capture detection. Journal of Chromatography A, 1216, 6741-6745.

Montes-Bayón, M., Díaz-Molet, M. J., Blanco-González, E. \& Sanz-Medel, A. (2006). Evaluation of different sample extraction strategies for selenium determination in selenium-enriched plants (Allium sativum and Brassica juncea) and Se speciation by HPLC-ICP-MS. Talanta, 68, 1287-1293.

Morales-Muñoz, S. \& Luque de Castro, M. D. (2005). Dynamic ultrasound-assisted extraction of colistin from feeds with on-line pre-column derivatization and liquid chromatographyfluorimetric detection. Journal of Chromatography A, 1066, 1-7.

Moreda-Piñeiro, A., Peña-Vázquez, E., Hermelo-Herbello, P., Bermejo-Barrera, P., Moreda-Piñeiro, J., Alonso-Rodríguez, E., Muniategui-Lorenzo, S., López-Mahía, P. \& Prada-Rodríguez, D. (2008). Matrix solid-phase dispersion as a sample pretreatment for the speciation of arsenic in seafood products. Analytical Chemistry, 80, 9272-9278.

Moreda-Piñeiro, J., Alonso-Rodríguez, E., López-Mahía, P., Muniategui-Lorenzo, S., Prada-Rodríguez, D., Romarís-Hortas, V., Míguez-Framil, M., Moreda-Piñeiro, A. \& Bermejo-Barrera, P. (2009). Matrix solid-phase dispersión of organic compounds and its feasibility for extracting inorganic and organometallic compounds. TrAC Trends in Analytical Chemistry, 28, 110-116.

Moreda-Piñeiro, J., Alonso-Rodriguez, E., Romaris-Hortas, V., Moreda-Pineiro, A., Lopez-Mahia, P., Muniategui-Lorenzo, S., Prada-Rodriguez, D. \& Bermejo-Barrera, P. (2012). Assessment of the bioavailability of toxic and non-toxic arsenic species in seafood samples. Food Chemistry, 130, 552-560.

Moreno, P., Quijano, M. A., Gutiérrez, A. M., Pérez-Conde, M. C. \& Cámara, C. (2001). Fractionation studies of selenium compounds from oysters, and their determination by high-performance liquid chromatography coupled to inductively coupled plasma mass spectrometry. Journal of Analytical Atomic Spectrometry, 16, 1044-1050.

Müller, I. (1998). Digestion and preparation of organic and biological microsamples for ultratrace elemental analysis. Atomic Spectroscopy, 19, 45-47. 
Müller, L. (1999). Field Analysis by SPME, in Pawliszyn, J. (Ed.), Applications of solid phase microextraction (pp. 269-283). Cambridge: Royal Society of Chemistry.

Müller-Esterl, W. (2008). Bioquímica. Fundamentos para medicina y ciencias de la vida. Editorial Reverté, S. A.: Barcelona.

Muñoz, A. H. S., Kubachka, K., Wrobel, K., Gutierrez-Corona, F., Yathavakill, S. K. V., Caruso, J. A. \& Wrobel, K. (2005). Metallomics approach to trace element analysis in Ustilago maydis using cellular fractionation, atomic absorption spectrometry, and size exclusion chromatography with ICP-MS detection. Journal of Agricultural and Food Chemistry, 53, 5138-5143.

Muñoz, J., Gallego, M. \& Valcárcel, M. (2005). Speciation of organometallic compounds in environmental samples by gas chromatography after flow preconcentration on fullerenes and nanotubes, Analytical Chemistry, 77, 5389-5395.

Muñoz-Ortuño, M., Moliner-Martínez, Y., Cogollos-Costa, S., Herráez-Hernández, R. \& CampínsFalcó, P. (2012). A miniaturized method for estimating di(2-ethylhexyl) phthalate in bivalves as bioindicators. Journal of Chromatography A, 1260, 169-173.

Muránszky, G., Óvári, M., Virág, I., Csiba, P., Dobai, R. \& Záray, G. (2011). Chemical characterization of $\mathrm{PM}_{10}$ fractions of urban aerosol. Microchemical Journal, 98, 1-10.

Naeeni, M. H., Yamini, Y. \& Rezaee, M. (2011). Combination of supercritical fluid extraction with dispersive liquid-liquid microextraction for extraction of organophosphorus pesticides from soil and marine sediment samples. Journal of Supercritical Fluids, 57, 219-226.

Nakazato, T., Akasaka, M. \& Tao, H. (2006). A rapid fractionation method for heavy metals in soil by continuous-flow sequential extraction assisted by focused microwaves. Analytical and Bioanalytical Chemistry, 386, 1515-1523.

Neves, R. C. F., Moraes, P. M., Saleh, M. A.D., Loureiro, V. R., Silva, F. A., Barros, M. M., Padilha, C.C. F., Jorge, S. M. A. \& Padilha, P. M. (2009). FAAS determination of metal nutrients in fish feed after ultrasound extraction. Food Chemistry, 113, 679-683.

Niemelä, M., Kola, H., Eilola, K. \& Perämäki, P. (2004). Development of analytical methods for the determination of sub-ppm concentrations of palladium and iron in methotrexate. Journal of Pharmaceutical and Biomedical Analysis, 35, 433-439.

Nieuwenhuize, J., Poley-Vos, C. H., van den Akker, A. H. \& van Delft, W. (1991). Comparison of microwave and conventional extraction techniques for the determination of metals in soil, sediment and sludge samples by atomic spectrometry. The Analyst, 116, 347-351.

Nóbrega, J. A., Santos, M. C., de Sousa, R. A., Cadore, S., Barnes, R. M. \& Tatro, M. (2006). Sample preparation in alkaline media. Spectrochimica Acta Part B: Atomic Spectroscopy, 61, 465-495.

Osberghaus, U. \& Helmers, E. (1997). Waste. In M. Stoeppler (Ed.) Sampling and sample preparation (pp. 57-74). Germany, Springer-Verlag Berlin Heidelberg.

Ozbek, N. \& Akman, S. (2012). A slurry sampling method for the determination of iron and zinc in baby food by flame atomic absorption spectrometry. Food Additives and Contaminants, 29, 208-216.

Öztan, S., Düring, R.- A. (2012). Microwave assisted EDTA extraction-determination of pseudototal contents of distinct trace elements in solid environmental matrices, Talanta, 99, 594-602.

Palacios, M. A., Varga, A., Gómez, M., Cámara C. \& Gavilanes, F. (1999). Evaluation of acid hydrolysis of proteins on Se-amino acids and trimethylselenonium species by liquid chromatographymicrowave digestion - hydride generation-atomic absorption spectrometry. Quimica Analítica, $18,163-168$

Pawliszyn, J. \& Lord, H. L (2010). Handbook of Sample Preparation. New Jersey: Wiley.

Pawliszyn, J. (2002). Sampling and sample preparation for field and laboratory. Netherlands: Elsevier.

Pawliszyn, J. (2003). Sample Preparation: Quo Vadis? Analytical Chemistry, 75, 2543-2558.

Pedrero, Z., Ruiz-Encinar, J., Madrid, Y. \& Cámara, C. (2007). Identification of selenium species in selenium-enriched Lens esculenta plants by using two-dimensional liquid chromatography- 
inductively coupled plasma mass spectrometry and $\left[{ }^{77} \mathrm{Se}\right]$ selenomethionine selenium oxide spikes. Journal of Chromatography A, 1139, 247-253.

Pellati, F., Pio Prencipe P., Bertelli, D. \& Benvenuti, S. (2013). An efficient chemical analysis of phenolic acids and flavonoids in raw propolis by microwave-assisted extraction combined with high-performance liquid chromatography using the fused-core technology. Journal of Pharmaceutical and Biomedical Analysis, 81-82, 126-132.

Pena-Abaurrea, M., García de la Torre, V. S. \& Ramos, L. (2013a). Ultrasound-assisted extraction followed by disposable pipette purification for the determination of polychlorinated biphenyls in small-size biological tissue samples. Journal of Chromatography A, 1317, 223-229.

Pena-Abaurrea, M., Ramos, J. J., Gonzalez, M. J. \& Ramos, L. (2013b). Miniaturized selective pressurized liquid extraction of polychlorinated biphenyls and polybrominateddiphenyl ethers from feedstuffs. Journal of Chromatography A, 1273, 18-25.

Peña-Farfal, C., Moreda-Piñeiro, A., Bermejo-Barrera, A., Bermejo-Barrera, P., Pinochet-Cancino, H. \& de Gregori-Henríquez, I. (2004). Ultrasound bath-assisted enzymatic hydrolysis procedures as sample pretreatment for the multielement determination in mussels by inductively coupled plasma atomic emission spectrometry. Analytical Chemistry, 76, 3541-3547.

Pena-Pereira, F., Lavilla, I. \& Bendicho, C. (2009). Miniaturized preconcentration methods based on liquid-liquid extraction and their application in inorganic ultratrace analysis and speciation: $\mathrm{A}$ review. Spectrochimica Acta Part B: Atomic Spectroscopy, 64, 1-15.

Pena-Pereira, F., Lavilla, I. \& Bendicho, C. (2010a). Liquid-phase microextraction techniques within the framework of green chemistry. TrAC Trends in Analytical Chemistry, 29, 617-628.

Pena-Pereira, F., Lavilla, I. \& Bendicho, C. (2010b). Liquid-phase microextraction approaches combined with atomic detection: A critical review. Analytica Chimica Acta, 669, 1-16.

Pena-Pereira, F., Lavilla, I. \& Bendicho, C. (2010c). Colorimetric assay for determination of trimethylamine-nitrogen (TMA-N) in fish by combining headspace-single-drop microextraction and microvolume UV-vis spectrophotometry. Food Chemistry, 119, 402-407.

Pérez, G., Valiente, M. \& Bendicho, C. (2008). A comparative study of metal readsorption in the application of a three-stage sequential extraction scheme and two accelerated versions (ultrasonic and single extractions). The Open Analytical Chemistry Journal, 2, 40-46.

Pérez-Cid, B. \& Boia, C. (2001). Comparison between total determination and extractable heavy metals from river sediments using conventional and microwave accelerated leaching tests. International Journal of Environmental Analytical Chemistry, 81, 101-115.

Pérez-Cid, B., Lavilla, I. \& Bendicho, C. (1998). Speeding up of a three-stage sequential extraction method for metal speciation using focused ultrasound. Analytica Chimica Acta, 360, 35-41.

Pérez-Cid, B., Lavilla, I. \& Bendicho, C. (1999). Application of microwave extraction for partitioning of heavy metals in sewage sludge. Analytica Chimica Acta, 378, 201-210.

Petrovic, M. \& Barceló, D. (2004) Environmental organic analytes. In J. M. Rosenfeld (Ed.) Sample preparation for hyphenated analytical techniques (pp.150-172). U.S. and Canada, Oxford: Blackwell.

Pino, V., Ayala, J. H., González, V. \& Afonso, A. M. (2013). Focused microwave-assisted micellar extraction combined with solid-phase microextraction-gas chromatography/mass spectrometry to determine chlorophenols in wood samples. Analytica Chimica Acta, 582, 10-18.

Pirsaheb, M., Fattahi, N. \& Shamsipur, M. (2013). Determination of organophosphorous pesticides in summer crops using ultrasound-assisted solvent extraction followed by dispersive liquid-liquid microextraction based on the solidification of floating organic drop. Food Control, 34, 378-385.

Pisonero, J., Fernández, B., Pereiro, R., Bordel, N. \& Sanz-Medel, A. (2006). Glow-discharge spectrometry for direct analysis of thin and ultra-thin solid films. TrAC Trends in Analytical Chemistry, 25, 11-18. 
Plata, M. R., Hernando, J., Zougagh, M., Contento, A. M., Villaseñor, M. J., Sánchez-Rojas \& J. L., Ríos, A. (2008). Characterization and analytical validation of a microcantilever-based sensor for the determination of total carbonate in soil samples, Sensors and Actuators B, 134, 245-251.

Pontes, F. V. M., Mendes, B. A. D., de Souza, E. M. F., Ferreira, F. N., da Silva, L.I. D., Carneiro, M. C., Monteiro, M. I. C., de Almeida, M. D., Neto, A. A. \& Vaitsman, D. S. (2010). Determination of metals in coal fly ashes using ultrasound-assisted digestion followed by inductively coupled plasma optical emission spectrometry. Analytica Chimica Acta, 659, 55-59.

Portet-Koltalo, F., Oukebdane, K., Dionner, F. \& Desbène, P. L. (2009). Optimisation of supercritical fluid extraction of polycyclic aromatic hydrocarbons and their nitrated derivatives adsorbed on highly sorptive diesel particulate matter. Analytica Chimica Acta, 28, 48-56.

Potts, P. J. \& Robinson, P. (2003). Sample preparation of geological samples, soils and sediments. In Z. Mester, R. Sturgeon (Eds.) Sample preparation for trace element analysis (pp. 723-764), Amsterdam, Elsevier.

Pourmortazavi, S. M., Rahimi-Nasrabadi \& M., Hajimirsadeghi, S. S. (2014). Supercritical fluid technology in analytical chemistry- Review. Current Analytical Chemistry, 10, 3-28.

Priego-Capote, F. \& Luque de Castro, M, (2007). Ultrasound in analytical chemistry. Analytical and Bioanalytical Chemistry, 387, 249-257.

Pu, Q., Sun, Q., Hu, Z. \& Su, Z. (1998). Application of 2-mercaptobenzothiazole-modified silica gel to on-line preconcentration and separation of silver for its atomic absorption spectrometric determination. The Analyst, 123, 239-243.

Pumure, I., Renton, J. J. \& Smart R. B. (2009). Accelerated aqueous leaching of selenium and arsenic from coal associated rock samples with selenium speciation using ultrasound extraction. Environmental Geology, 56, 985-991.

Puttemans, F., van den Winkel, P. \& Massart D. L. (1983). The determination of arsenic by electrothermal atomic absorption spectrometry after liquid-liqiud extraction. Analytical Chimica Acta, $149,123-128$.

Qiao, F. \& Sun, H. (2010). Simultaneous extraction of enrofloxacin and ciprofloxacin from chicken tissue bymolecularly imprinted matrix solid-phase dispersion. Journal of Pharmaceutical and Biomedical Analysis, 53, 795-798.

Qiao, F., \& Yan, H. (2010). Simultaneous analysis of fluoroquinolones and xanthine derivatives in serum by molecularly imprinted matrix solid-phase dispersion coupled with liquid chromatography, Journal of Chromatography B: Analytical Technologies in the Biomedical and Life Sciences, 879, 3551-3555.

Quevauviller, P., Rauret, G. \& Griepink, B. (1993). Single and sequential extraction in sediments and soils. International Journal of Environmental Analytical Chemistry, 51, 231-235.

Quevauviller, P., Rauret, G., Rubio, R., López-Sánchez, J.-F., Ure, A., Bacon, J. \& Muntau, H. (1997). Certified reference materials for the quality control of EDTA- and acetic acid-extractable contents of trace elements in sewage sludge amended soils (CRMs 483 and 484). Fresenius Journal of Analytical Chemistry, 357, 611-618.

Quijano, M. A., Moreno, P., Gutiérrez, A. M., Pérez-Conde, M. C. \& Cámara, C. (2000). Selenium speciation in animal tissues after enzymatic digestion by high-performance liquid chromatography coupled to inductively coupled plasma mass spectrometry. Journal of Mass Spectrometry, 35, 878-884.

Quintana, J. B., Boonjob, W., Miró, M., Cerda, V. (2009). Online coupling of bead injection lab-on-valve analysis to gas chromatography: application to the determination of trace levels of polychlorinated biphenyls in solid waste leachates. Analytical Chemistry, 81, 4822-4830.

Ramalhosa, E., Río-Segade, S., Pereira, E., Vale, C. \& Duarte, A. (2001). Microwave-assisted extraction for methylmercury determination in sediments by high performance liquid chromatography-cold vapour atomic fluorescence spectrometry. Journal of Analytical Atomic Spectrometry, 16, 643-647. 
Ramalhosa, M. J., Paíga, P., Morais, S., Sousa, A. M. M., Gonçalves, M. P., Delerue-Matos, C. \& Prior Pinto Oliveira, M. B. (2012). Analysis of polycyclic aromatic hydrocarbons in fish: Optimisation and validation of microwave-assisted extraction. Food Chemistry, 135, 234-242.

Ramil-Criado, M., Rodríguez-Pereiro, I. \& Cela-Torrijos, R. (2004). Determination of polychlorinated biphenyls in ash using dimethylsulfoxide microwave assisted extraction followed by solid-phase microextraction. Talanta, 63, 533-540.

Ramos, J. J., Dietz, C., Gonzalez, M. J. \& Ramos, L. (2000). Miniaturised selective pressurised liquid extraction of polychlorinated biphenyls from foodstuffs. Journal of Chromatography A, 1152, 254-261.

Ramos, J. J., Rial-Otero, R., Ramos, L. \& Capelo, J. L. (2008). Ultrasonic-assisted matrix solid-phase dispersion as an improved methodology for the determination of pesticides in fruits. Journal of Chromatography $A, 1212,145-149$.

Ramos, L. (2012). Critical overview of selected contemporary sample preparation techniques. Journal of Chromatography A, 1221, 84- 98.

Ramos, L., Ramos, J. J. \& Brinkman, U. A. T. (2005). Miniaturization in sample treatment for environmental analysis. Analytical and Bioanalytical Chemistry, 381, 119-140.

Ramos, L., Vreuls, J. J. \& Brinkman, U. A. Th. (2007). Miniaturised pressurised liquid extraction of polycyclic aromatic hydrocarbons from soil and sediment with subsequent large-volume injection-gas chromatography. Journal of Chromatography A, 891, 275-286.

Rasmussen, R. R., Hedegaard, R. V., Larsen, E. H. \& Sloth, J. J. (2012). Development and validation of an SPE HG-AAS method for determination of inorganic arsenic in samples of marine origin. Analytical and Bioanalytical Chemistry, 403, 2825-2834.

Ravelo-Pérez, L. M., Herrera-Herrera, A. V., Hernández-Borges, J. \& Rodríguez-Delgado, M. A. (2010). Carbon nanotubes: Solid-phase extraction. Journal of Chromatography A, 1217, 2618-2641.

Rechenberg, W. (1997). Decomposition [of materials] for trace analysis in the cement industry. In M. Stoeppler (Ed.) Sampling and sample preparation (pp. 183-198). Germany: Springer-Verlag Berlin Heidelberg.

Reid, M. K., Spencer, K. L. \& Shotbolt, L. (2011). An appraisal of microwave-assisted Tessier and BCR sequential extraction methods for the analysis of metals in sediments and soils. Journal of Soils and Sediments, 11, 518-528.

Remeteiová, D., Ružičková, S. \& Rusnák, R. (2008). Ultrasound-assisted extraction in the fractionation analysis, of gravitation dust sediments. Microchimica Acta, 163, 257-261.

Reus, U. (1991). Determination of trace elements in oils and greases with total reflection X-ray fluorescence: sample preparation methods. Spectrochimica Acta Part B: Atomic Spectroscopy, 46, 1403-1411.

Reyes, L. H., Rahman, G. M. M., Fahrenholz, T. \& Kingston, H. M. S. (2008). Comparison of methods with respect to efficiencies, recoveries, and quantitation of mercury species interconversions in food demonstrated using tuna fish. Analytical and Bioanalytical Chemistry, 390, 2123-2132.

Reyes-Garcés, N., Gómez-Ríos, G. A., Souza Silva, É. A., \& Pawliszyn, J. (2013). Coupling needle trap devices with gas chromatography-ion mobility spectrometry detection as a simple approach for on-site quantitative analysis. Journal of Chromatography A, 1300 193-198.

Rezaei, F. \& Hosseini, M. (2011). New method based on combining ultrasonic assisted miniaturized matrix solid-phase dispersion and homogeneous liquid-liquid extraction for the determination of some organochlorinated pesticides in fish. Analytica Chimica Acta, 702, 274-279.

Ribeiro, A. S., Curtius, A. J. \& Pozebon, D. (2000). Determination of As, Cd, Ni and Pb in human hair by electrothermal atomic absorption spectrometry after sample treatment with tetramethylammonium hydroxide. Microchemical Journal, 64, 105-110.

Richter, B. R., Jones, B. A., Ezzell, J. L., Porter, N. L., Avdalovic, N. \& Pohl, C. (1996.) Accelerated solvent extraction: a technique for sample preparation. Analytical Chemistry, 68, 1033-1039. 
Ríos, A. \& Zougagh, M. (2013). Sample preparation for micro total analytical systems ( $\mu$-TASs). TrAC Trends in Analytical Chemistry, 43, 174-188.

Ríos, A., Escarpa, A., Simonet, B. (2009). Miniaturization Systems: Principles, designs and applications. United Kingdom: John Wiley \& Sons.

Ríos, A., Zougagh, M. \& Avila, M. (2012). Miniaturization through lab-on-a-chip: Utopia or reality for routine laboratories? A review. Analytica Chimica Acta, 740, 1-11.

Rodríguez, I., Santamarina, M., Bollaín, M. H., Mejuto, M. C. \& Cela, R. (1997). Speciation of organotin compounds in marine biomaterials after basic leaching in a non-focused microwave extractor equipped with pressurized vessels. Journal of Chromatography A, 774, 379-387.

Romero, R., Costas-Mora, I., Lavilla, I. \& Bendicho, C. (2011). Cold vapor-solid phase microextraction using amalgamation in different $\mathrm{Pd}$-based substrates combined with direct thermal desorption in a modified absorption cell for the determination of $\mathrm{Hg}$ by atomic absorption spectrometry. Spectrochimica Acta Part B: Atomic Spectroscopy, 66, 156-162.

Russo R. E. \& Baldwin D. P. (2003). Laser ablation sampling. In Z. Mester \& R. Sturgeon (Eds.), Sample preparation for trace element analysis (pp. 593-609). The Netherlands: Elsevier.

Salvador, A., Pascual-Martí, M. C., Adell, J. R., Requeni, A. \& March, J. G. (2000). Analytical methodologies for atomic spectrometric determination of metallic oxides in UV sunscreen creams. Journal of Pharmaceutical and Biomedical Analysis, 22, 301-306.

Sánchez-Moreno, R. A., Gismera, M. J., Sevilla, M. T. \& Procopio, J. R. (2010). Direct and rapid determination of ultratrace heavy metals in solid plant materials by ET-AAS ultrasonic-assisted slurry sampling. Phytochemical Analysis, 21, 340-347.

Sansoni, B. \& Panday V. K. (1994) Trace element analysis in biological specimens. In R. F. M. Herber \& M. Stoeppler (Ed.) Sample treatment of human biological materials, (pp. 21-52). The Netherlands: Elsevier.

Santos, C., Alava-Moreno, F., Lavilla, I. \& Bendicho, C. (2000). Total As in seafood as determined by transverse heated electrothermal atomic absorption spectrometry-longitudinal Zeeman background correction: An evaluation of automated ultrasonic slurry sampling, ultrasoundassisted extraction and microwave-assisted digestion methods. Journal of Anaytical Atomic Spectrometry, 15, 987-994.

Santos-Júnior, D., Krug, F. J., de Godoi-Pereira, M. \& Korn, M. (2006). Currents on ultrasoundassisted extraction for sample preparation and spectroscopic analytes determination. Applied Spectroscopy Reviews, 41, 305-321.

Sanz, E., Muñoz-Olivas, R. \& Cámara, C. (2005a). Evaluation of a focused sonication probe for arsenic speciation in environmental and biological samples. Journal of Chromatography $A$, 1097, 1-8.

Sanz, E., Muñoz-Olivas, R. \& Cámara, C. (2005b). A rapid and novel alternative to conventional sample treatment for arsenic speciation in rice using enzymatic ultrasonic probe. Analytica Chimica Acta, 535, 227-235.

Sanz, E., Muñoz-Olivas, R., Dietz, C., Sanz, J. \& Cámara, C. (2007) Alternative extraction methods for arsenic speciation in hair using ultrasound probe sonication and pressurised liquid extraction. Journal of Anaytical Atomic Spectrometry, 22, 131-139.

Sanz-Segundo, C., Hernández-Artiga, M. P., Hidalgo-Hidalgo de Cisneros, J. L., Bellido-Milla, D. \& Naranjo-Rodriguez, I. (1999). Determination of wear metals in marine lubricating oils by microwave digestion and atomic absorption spectrometry. Microchimica Acta, 132, 89-94.

Sávoly, Z., Nagy, P., Havancsák, K. \& Záray, G. (2012). Microanalytical investigation of nematodes. Microchemical Journal, 105, 83-87.

Scaccia, S. \& Mecozzi, R. (2012). Trace Cd, Co, and Pb elements distribution during Sulcis coal pyrolysis: GFAAS determination with slurry sampling technique. Microchemical Journal, 100, 48-54. 
Schladot, J.-D. \& Backhaus, F. (1997). Collection, preparation and long-term storage of marine samples. In M. Stoeppler (Ed.) Sampling and sample preparation (pp. 74-87). Germany: Springer-Verlag Berlin Heidelberg.

Schmeling, M., Klockenkämper, R. \& Klockow, D. (1997). Application of total-reflection X-ray fluorescence spectrometry to the analysis of airborne particulate matter. Spectrochimica Acta Part B: Atomic Spectroscopy, 52, 985-994.

Schöniger, W. (1955). Rapid microanalytical determination of halogen in organic compounds. Microchimica Acta, 1, 123-129.

Schöniger, W. (1956). The rapid microanalytical determination of halogens and sulfur in organic substances Microchimica Acta, 1, 869-876.

Seidi, S., Yamini, Y., Heydari, A., Moradi, M., Esrafili, A. \& Rezazadeh, M. (2011). Determination of the baine in water samples, biological fluids, poppy capsule, and narcotic drugs, using electromembrane extraction followed by high-performance liquid chromatography analysis. Analytica Chimica Acta, 701, 181-188.

Self, R. (2005). Extraction of organic analytes from foods. London: Royal Society of Chemistry.

Sereshti, H., Bakhtiari, S. \& Khojeh, V. (2011). Simultaneous determination of aluminum, copper, iron and zinc in Oscillatoria, Juncus littoralis tissues and wetland water samples by ultrasoundassisted emulsification-microextraction combined with ICP-OES. Analytical Methods, 3, 2936-2942.

Sereshti, H., Heidari, R. \& Samadi, S. (2014). Determination of volatile components of saffron by optimised ultrasound-assisted extraction in tandem with dispersive liquid-liquid microextraction followed by gas chromatography-mass spectrometry. Food Chemistry, 143, 499-505.

Shamsipur, M., Ramezani, M. \& Sadeghi, M. (2009). Preconcentration and determination of ultra trace amounts of palladium in water samples by dispersive liquid-liquid microextraction and graphite furnace atomic absorption spectrometry. Microchimica Acta, 166, 235-242.

Shen, Q., Dong, W., Wang, Y., Gong, L., Dai, Z. \& Cheung, H. (2013a). Pipette tip solid-phase extraction and ultra-performance liquid chromatography/mass spectrometry based rapid analysis of picrosides from Picrorhizascrophularii flora. Journal of Pharmaceutical and Biomedical Analysis, 136-140.

Shen, Q., Gong, L., Baibado, J. T., Dong, W., Wang, Y., Dai, Z. \& Cheung, H. (2013b). Graphene based pipette tip solid phase extraction of marine toxins in shellfish muscle followed by UPLC-MS/MS analysis. Talanta, 116, 770-775.

Shiddiky, M. J. A., Lee, K.-S., Son, J., Park, D.-S. \& Shim, Y.-B. (2009). Development of extraction and analytical methods of nitrite ion from food samples: Microchip electrophoresis with a modified electrode. Journal of Agricultural Food Chemistry, 57, 4051-4057.

Sides, S. L., Polowy, K. B., Thornquest Jr., A. D., \& Burinsky, D. J. (2001). Identification of a pharmaceutical packaging off-odor using solid phase microextraction gas chromatography/ mass spectrometry. Journal of Pharmaceutical and Biomedical Analysis, 25, 379-386.

Silva, R. G. L., Willie, S. N., Sturgeon, R. E., Santelli, R. E. \& Sella, S. M. (1999). Alkaline solubilization of biological materials for trace element analysis by electrothermal atomic absorption spectrometry. The Analyst, 124, 1843-1846.

Siwek, M., Noubar, A. B., Bergmann, J., Niemeyer, B. \& Galunsky, B. (2006). Enhancement of enzymatic digestion of Antarctic krill and successive extraction of selenium organic compounds by ultrasound treatment. Analytical and Bioanalytical. Chemistry, 384, 244-249.

Skorek, R., Turek, E., Zawisza, B., Marguí, E., Queralt, I., Stempin, M., Kucharski, P. \& Sitko, R. (2012). Determination of selenium by $\mathrm{X}$-ray fluorescence spectrometry using dispersive solid-phase microextraction with multiwalled carbon nanotubes as solid sorbent. Journal of Analytical Atomic Spectrometry, 27, 1688-1693.

Smith, F. E. \& Arsenault, E. A. (1996). Microwave-assisted sample preparation in analytical chemistry. Talanta, 43, 1207-1268. 
Solchaga, M., Montoro, R. \& de la Guardia, M. (1986). Flame absorption spectroscopic determination of cadmium, copper, iron, lead, and zinc in mussels. Journal of the Association of Official Analytical Chemistry, 69, 874-876.

Song, W., Zhang, Y., Li, G., Chen, H., Wang, H., Zhao, Q., He, D., Zhao, C. \& Ding, L. (2014). A fast, simple and green method for the extraction of carbamate pesticides from rice by microwave assisted steam extraction coupled with solid phase extraction. Food Chemistry, 143, 192-198.

Song, X., Shi, Y. \& Chen, J. (2013). Carbon nanotubes-reinforced hollow fibre solid-phase microextraction coupled with high performance liquid chromatography for the determination of carbamate pesticides in apples. Food Chemistry, 139, 246-252,

Souza Silva, E. A., Risticevic, S. \& Pawliszyn, J. (2013). Recent trends in SPME concerning sorbent materials, configurations and in vivo applications. TrAC Trends in Analytical Chemistry, 43, 24-36.

Soylak, M., Tuzen, M., Narin, I. \& Sari, H. (2004). Comparison of microwave, dry and wet digestion procedures for the determination of trace metal contents in spice samples produced in Turkey. Journal of Food and Drug Analysis, 12, 254-258.

Spanke, J., von Bohlen, A., Klockenkamper, R., Quentmeier, A. \& Klockow, D. (2000). Total reflection $\mathrm{X}$-ray fluorescence analysis of laser-deposited solid sample material. Journal of Analytical Atomic Spectrometry, 15, 673-679.

Starr, J. M. \& Selim, M. I. (2008). Supercritical fluid extraction of aflatoxin B from soil. Journal of Chromatography A, 1209, 37-43.

Stashenko, E.E., Ordoñez, S. A., Marin, N. A. \& Martinez, J. R. (2009). Determination of the volatile and semi-volatile secondary metabolites, and aristolochic acids in Aristolochiaringens Vahl. Journal of Chromatographic Science, 47, 817-821.

Steiner J. W., Moy, D. C., Kramer, H. L. (1987). Rapid determination of chromium in bovine liver using an atomic absorption spectrometer with a modified carbon rod atomizer. The Analyst, 112, $1113-1115$.

Stoeppler, M. (1997). Sampling: an introduction. In M. Stoeppler (Ed.) Sampling and sample preparation (pp. 1-6). Germany: Springer-Verlag Berlin Heidelberg.

Su, R., Wang, X., Xu, X., Wang, Z., Li, D., Zhao, X., Li, X., Zhang, H. \& Yu, A. (2011). Application of multiwall carbon nanotubes-based matrix solid phase dispersion extraction for determination of hormones in butter by gas chromatography mass spectrometry. Journal of Chromatography A, 1218, 5047-5054.

Subramanian, K. S. (1996). Determination of metals in biofluids and tissues: sample preparation methods for atomic spectroscopic techniques. Spectrochimica Acta Part B: Atomic Spectroscopy, 51, 291-319.

Sun, Y. \& Li, H. (2000) Determination of trace selenium in human plasma and hair with ternary inclusion compound-fluorescent spectrophotometry. The Analyst, 125, 2326-2329.

Sunarso, J. \& Ismadji, S. (2009). Decontamination of hazardous substances from solid matrices and liquids using supercritical fluids extraction: A review. Journal of Hazardous Materials, 161, 1-20.

Sutherland, R. A. (2010). BCR®-701: A review of 10-years of sequential extraction analyses, Analytica Chimica Acta, 680, 10-20.

Szoboszlai, N., Réti, A., Budai, B., Szabó, Z., Kralovánszky, J. \& Záray, G. (2008). Direct elemental analysis of cancer cell lines by total reflection X-ray fluorescence. Spectrochimica Acta Part B: Atomic Spectroscopy, 63, 1480-1484.

Szpunar, J., Bouyssiere, B. \& Lobinski, R. (2003). Sample preparation for speciation analysis for metallobiomolecules, In Z. Mester \& R. Sturgeon (Eds.), Sample preparation for trace element analysis (pp. 1185-1210). The Netherlands: Elsevier.

Szpunar, J., Schmitt, V. O., Donard, O. F. X. \& Lobinski, R. (1996). Low-power focused microwave technology as a new tool for rapid preparation of solid samples for speciation analysis. TrAC Trends in Analytical Chemistry, 15, 181-187. 
Talukder, M. M. R., Zaman, M. M., Hayashi, Y., Wu, J. C. \& Kawanishi, T. (2006). Ultrasonication enhanced hydrolytic activity of lipase in water/isooctane two-phase systems. Biocatalysis and Biotransformation, 24, 189-194.

Tan, C.S., Oliveira, E., Oliveira, P.V., Abstracts of 11 Encontro Nacional de Química Analítica, Campinas, SP, 2001.

Tan, Y. \& Marshall, W. D. (1997). Enzymic Digestion-High-pressure Homogenization Prior to Slurry Introduction Electrothermal Atomic Absorption Spectrometry for the Determination of Selenium in Plant and Animal Tissues. The Analyst, 122, 13-18.

Teixeira-Tarley, C. R., Barbosa, A. F., Segatelli, M. G., Figueiredo, E. C. \& Luccas, P. O. (2006). Highly improved sensitivity of TS-FF-AAS for Cd(II) determination at $\mathrm{ng} \mathrm{L}-1$ levels using a simple flow injection minicolumn preconcentration system with multiwall carbon nanotubes. Journal of Analytical Atomic Spectrometry, 21, 1305-1313.

Tessier, A., Campbell, P. G. C. \& Bisson, M. (1979). Sequential extraction procedure for the speciation of particulate trace metals. Analytical Chemistry, 51, 844-851.

Theisen, M. \& Niessner, R. (1999). Elemental analysis of airborne dust samples with TXRF: Comparison of oxygen-plasma ashing on sapphire carriers and acid digestion for sample preparation. Fresenius Journal of Anaytical Chemistry, 365, 332-337.

Thomas, A. D. \& Smythe, L. E. (1973) Rapid destruction of plant material with concentrated nitric acid vapour (vapour phase oxidation). Talanta, 20, 469-475.

Togunde, O. P., Oakes, K. D., Servos, M. R. \& Pawliszyn, J. (2012). Optimization of solid phase microextraction for non-lethal in vivo determination of selected pharmaceuticals in fish muscle using liquid chromatography-mass spectrometry, Journal of Chromatography A, 1261, 99-106.

Tran, M. Q. T., Nygren, Y., Lundin, C., Naredi, P. \& Björn, E. (2010). Evaluation of cell lysis methods for platinum metallomic studies of human malignant cells. Analytical Biochemistry, 396, 76-82.

Trevizan, L. C., Nogueira, A. R. A. \& Nóbrega, J. A. (2003). Single vessel procedure for acid vapor partial digestion of bovine liver in a focused microwave: multielement determination by ICP-OES. Talanta, 61, 81-86.

Turner, A. \& Olsen, Y. S. (2000). Chemical versus enzymatic digestion of contaminated estuarine sediment: Relative importance of iron and manganese oxides in controlling trace metal bioavailability. Estuarine, Coastal and Shelf Science, 51, 717-728.

Turner, A. (2006). Enzymatic mobilisation of trace metals from estuarine sediment. Marine Chemistry, 98, 140-147.

Turner, A., Henon, D. N. \& Dale, J. L. L. (2001) Pepsin-digestibility of contaminated estuarine sediments. Estuarine, Coastal and Shelf Science, 53, 671-681.

Tuzen, M., Saygi, K. O. \& Soylak, M. (2008). Solid phase extraction of heavy metal ions in environmental samples on multiwalled carbon nanotubes. Journal of Hazardous Materials, 152, 632-639.

Umale, S. \& Mahanwar, P. A. (2010). Supercritical fluid extraction of heavy metals from solid matrices. Chemical Technology: An Indian Journal, 5, 25-44.

Väisänen, A. \& Kiljunen, A. (2005). Ultrasound-assisted sequential extraction method for the evaluation of mobility of toxic elements in contaminated soils. International Journal of Environmental Analytical Chemistry, 85, 1037-1049.

Väisänen, A., Suontamo, R., Silvonen, J. \& Rintala, J. (2002). Ultrasound-assisted extraction in the determination of arsenic, cadmium, copper, lead, and silver in contaminated soil samples by inductively coupled plasma atomic emission spectrometry. Analytical and Bioanalytical Chemistry, 373, 93-97.

Valcárcel, M., \& Cárdenas, M. S. (2000). Automatización y miniaturización en química analítica. Barcelona: Springer. 
Vale, G., Pereira, S., Mota, A., Fonseca, L. \& Capelo, J. L. (2007) Enzymatic probe sonication as a tool for solid-liquid extraction for total selenium determination by electrothermal-atomic absorption spectrometry. Talanta, 74, 198-205.

Vale, G., Rial-Otero, R., Mota, A., Fonseca, L. \& Capelo, J. L. (2008). Ultrasonic-assisted enzymatic digestion (USAED) for total elemental determination and elemental speciation: A tutorial. Talanta, 75, 872-884.

Vanhaecke, F., Resano, M. \& Moens, L. (2002). Electrothermal vaporisation ICP-mass spectrometry (ETV-ICP-MS) for the determination and speciation of trace elements in solid samples - A review of real-life applications from the author's lab. Analytical and Bioanalytical Chemistry, 374, 188-195.

Varga, I., Rierpl, E. \& Tusai, A. (1999). Comparison of slurry preparation and microwave digestion of freshwater algae for multi-element analysis by total reflection X-ray fluorescence spectrometry. Journal of Analytical Atomic Spectrometry, 14, 881-883.

Varga, I., Szebeni, A., Szoboszlai, N. \& Kovács, B. (2005). Determination of trace elements in human liver biopsy samples by ICP-MS and TXRF: hepatic steatosis and nickel accumulation. Analytical and Bioanalytical Chemistry, 383, 476-482.

Vázquez, C., Custo, G., Barrio, N., Burucúa, J., Boeykens, S. \& Marte, F. (2010). Inorganic pigment study of the San Pedro Gonzalez Telmo Sibyls using total reflection X-ray fluorescence. Spectrochimica Acta Part B: Atomic Spectroscopy, 65, 852-858.

Vazquez, M. J., Carro, A. M., Lorenzo, R. A. \& Cela, R. (1997). Optimization of methylmercury microwave-assisted extraction from aquatic sediments. Analytical Chemistry, 69, 221-225.

Veggi, P. C., Martinez, J. \& Meireles, M. A. A. (2013). Fundamentals of microwave extraction, in: Chemat, F. \& Cravotto, G. (Ed.), Microwave-assisted extraction for bioactive compounds, theory and practice (pp. 15-53). Netherlands: Springer.

Vignola, F., Borges, D. L. G., Curtius, A. J., Welz, B. \& Becker-Ross, H. (2010). Simultaneous determination of $\mathrm{Cd}$ and $\mathrm{Fe}$ in sewage sludge by high-resolution continuum source electrothermal atomic absorption spectrometry with slurry sampling. Microchemical Journal, 95 , 333-336.

Vilanó, M. \& Rubio, R. (2001). Determination of arsenic species in oyster tissue by microwaveassisted extraction and liquid chromatography-atomic fluorescence detection. Applied Organometallic Chemistry, 15, 658-666.

Viñas, P., Bravo-Bravo, M., López-García, I. \& Hernández-Córdoba, M. (2013). Dispersive liquid-liquid microextraction for the determination of vitamins $D$ and $K$ in foods by liquid chromatography with diode-array and atmospheric pressure chemical ionization-mass spectrometry detection. Talanta, 115, 806-813.

Wagner, A. \& Mages, M. (2010). Total-Reflection X-ray fluorescence analysis of elements in size-fractionated particulate matter sampled on polycarbonate filters - composition and sources of aerosol particles in Göteborg, Sweden. Spectrochimica Acta Part B: Atomic Spectroscopy, 65, 471-477.

Wang, H., Li, G., Zhang. Y., Chen, H., Zhao, Q., Song, W., Xu, Y., Jin, H. \& Ding, L. (2012). Determination of triazine herbicides in cereals using dynamic microwave-assisted extraction with solidification of floating organic drop followed by high-performance liquid chromatography. Journal of Chromatography A, 1233, 36-43.

Wang, H., So, P. \& Yao, Z. (2014). Direct analysis of herbal powders by pipette-tip electrospray ionization mass spectrometry. Analytica Chimica Acta, 809, 109-116.

Wang, H., Wu, Z., Zhang, Y., Chen, B., He, M. \& Hu, B. (2013a). Chip-based liquid phase microextraction combined with electrothermal vaporization-inductively coupled plasma mass spectrometry for trace metal determination in cell samples. Journal of Analytical Atomic Spectrometry, 28, 1660-1665. 
Wang, J. \& Hansen, E. H. (2001a). Coupling sequential injection on-line preconcentration by means of a renewable microcolumn with ion-exchange beads with detection by electrothermal atomic absorption spectrometry comparing the performance of eluting the loaded beads with transporting them directly into the graphite tube, as demonstrated for the determination of nickel in environmental and biological samples. Analytica Chimica Acta, 435, 331-342.

Wang, J. \& Hansen, E. H. (2001b). Interfacing sequential injection on-line preconcentration using a renewable micro-column incorporated in a 'lab-on-valve' system with direct injection nebulization inductively coupled plasma mass spectrometry. Journal of Analytical Atomic Spectrometry, 16, 1349-1355.

Wang, J., Hansen, E. H. \& Miró, M. (2003). Sequential injection-bead injection-lab-on-valve schemes for on-line solid phase extraction and preconcentration of ultra-trace levels of heavy metals with determination by electrothermal atomic absorption spectrometry and inductively coupled plasma mass spectrometry. Analytica Chimica Acta, 499, 139-147.

Wang, S., Lin, Y. \& Wai, C. M. (2003). Supercritical fluid extraction of toxic heavy metals from solid and aqueous matrices. Separation Science and Technology 38, 2279-2289.

Wang, X., Ding, L., Zhang, H., Cheng, J., Yu, A., Zhang, H., Liu, L., Liu, Z. \& Li, Y. (2006). Development of an analytical method for organotin compounds in fortified flour samples using microwaveassisted extraction and normal-phase HPLC with UV detection. Journal of Chromatography B: Analytical Technologies in the Biomedical and Life Sciences, 843, 268-274.

Wang, X., Jin, H., Ding, L., Zhang, H., Zhang, H., Qu, C. \& Yu, A. (2008). Organotin speciation in textile and plastics by microwave-assisted extraction HPLC-ESI-MS. Talanta, 75, 556-563.

Wang, Z., Zhao, X., Xu, X., Wu, L., Su. R., Zhao, Y., Jiang, C., Zhang, H., Ma, Q., Lu, C. \& Dong, D. (2013b). An absorbing microwave micro-solid-phase extraction device used in non-polar solvent microwave-assisted extraction for the determination of organophosphorus pesticides. Analyica Chimica Acta, 760, 60-68.

Welz, B., Sperling, M. (1999). Atomic absorption spectrometry, Germany: Wiley-VCH.

Welz, B., Vale, M. G. R., Borges, D. L. G. \& Heitmann, U. (2007). Progress in direct solid sampling analysis using line source and high-resolution continuum source electrothermal atomic absorption spectrometry. Analytical and Bioanalytical Chemistry, 389, 2085-2095.

Wieteska, E., Zióek, A. \& Drzewihska, A. (1996). Extraction as a method for preparation of vegetable samples for the determination of trace metals by atomic absorption spectrometry, Analytica Chimica Acta, 330, 25 l-257.

Woelfl, S., Mages, M. \& Encina, F. (2003). Cold plasma ashing improves the trace element detection of single Daphnia specimens by total reflection X-ray fluorescence spectrometry. Spectrochimica Acta Part B: Atomic Spectroscopy, 58, 2157-2168.

Woelfl, S., Mages, M., Mercado, S., Villalobos, L. \& Encina, M. O. F. (2004). Determination of trace elements in planktonic microcrustaceans using total reflection X-ray fluorescence (TXRF): first results from two Chilean lakes. Analytica Bioanalytical Chemistry, 378, 1088-1094.

Wu, S. \& Ding, W. (2010). Fast determination of synthetic polycyclic musks in sewage sludge and sediments by microwave-assisted headspace solid-phase microextraction and gas chromatography-mass spectrometry. Journal of Chromatography A, 1217, 2776-2781.

Wu, S., Liu, L. \& Ding, W. (2012). One-step microwave-assisted headspace solid-phase microextraction for the rapid determination of synthetic polycyclic musks in oyster by gas chromatography-mass spectrometry. Food Chemistry, 133, 513-517.

Xi, Y., Duford, D. A. \& Salin, E. D. (2010). Automated liquid-solid extraction of pyrene from soil on centrifugal microfluidic devices. Talanta, 82, 1072-1076.

Xia, L., Wu, Y. \& Hu, B. (2007). Hollow-fiber liquid-phase microextraction prior to low-temperature electrothermal vaporization ICP-MS for trace element analysis in environmental and biological samples. Journal of Mass Spectrometry, 42, 803-810. 
Xu, Z. G., Hu, Y.F., Hu, Y. L. \& Li, G. K. (2010). Investigation of ractopamine molecularly imprinted stir bar sorptive extraction and its application for trace analysis of beta (2)-agonists in complex samples. Journal of Chromatography A, 1217, 3612-3618.

Xue, S., Liu, Y., Li, H.-F., Uchiyama, K. \& Lin, J. M. (2013). A microscale solid-phase extraction poly(dimethylsiloxane) chip for enrichment and fluorescent detection of metal ions. Talanta, 116, 1005-1009.

Yabalak, E. \& Gizir, A. M. (2013). Subcritical and supercritical fluid extraction of heavy metals from sand and sewage sludge. Journal of the Serbian Chemical Society, 78, 1013-1022.

Yamamoto, K. Y., Cremers, D. A., Ferris, M. J. \& Foster, L. E. (1996). Detection of metals in the environment using a portable laser-induced breakdown spectroscopy instrument. Applied Spectroscopy, 50, 222-233.

Yan, H., Cheng, X., Sun, N., Cai, T., Wu, R. \& Han, K. (2012a). Rapid and selective screening of melamine in bovine milk using molecularly imprinted matrix solid-phase dispersion coupled with liquid chromatography-ultraviolet detection. Journal of Chromatography B: Analytical Technologies in the Biomedical and Life Sciences, 908, 137-142.

Yan, H., Wang, F., Wang, H. \& Yang, G. (2012b). Miniaturized molecularly imprinted matrix solid-phase dispersion coupled with high performance liquid chromatography for rapid determination of auxins in orange samples. Journal of Chromatography A, 1256, 1-8.

Yan, H., Wang, H., Qiao, J. \& Yang, G. (2011). Molecularly imprinted matrix solid-phase dispersion combined with dispersive liquid-liquid microextraction for the determination of four Sudan dyes in egg yolk, Journal of Chromatography A, 1218, 2182-2188.

Yang, G., Xu, J., Xu, L., Chen, G. \& Fu, F. (2010). Analysis of ultratrace triorganotin compounds in aquatic organisms by using capillary electrophoresis-inductively coupled plasma mass spectrometry. Talanta, 80, 1913-1918.

Yi, Y.-Z., Jiang, S.-J. \& Sahayam, A. C. (2012). Palladium nanoparticles as the modifier for the determination of $\mathrm{Zn}, \mathrm{As}, \mathrm{Cd}, \mathrm{Sb}, \mathrm{Hg}$ and $\mathrm{Pb}$ in biological samples by ultrasonic slurry sampling electrothermal vaporization inductively coupled plasma mass spectrometry. Journal of Analytical Atomic Spectrometry, 27, 426-431.

Yin, X., Frech, W., Hoffmann, E., Lüdke, C. \& Skole, J. (1998). Mercury speciation by coupling cold vapour atomic absorption spectrometry with flow injection on-line preconcentration and liquid chromatographic separation. Fresenius Journal of Analytical Chemistry, 361, 761-766.

Yu, Y., Chen, B., Shen, C., Cai, Y., Xie, M., Zhou, W., Chen, Y., Li, Y. \& Duan, G. (2010). Multiple headspace single-drop microextraction coupled with gas chromatography for direct determination of residual solvents in solid drug product. Journal of Chromatography A, 1217, 5158-5164.

Yu, Y.-L., Jiang, Y., Chen, M.-L. \& Wang, J.-H. (2011). Lab-on-valve in the miniaturization of analytical systems and sample processing for metal analysis. TrAC Trends in Analytical Chemistry, 30, 1649-1658.

Yu, Z., Robinson, P. \& McGoldrick, P. (2001). An evaluation of methods for the chemical decomposition of geological materials for trace element determination using ICP-MS. Geostandard Newsletter, 25, 199-217.

Zang, Z., Hu, Z., Li, Z., He, Q. \& Chang, X. (2009). Synthesis, characterization and application of ethylenediamine-modified multiwalled carbon nanotubes for selective solid-phase extraction and preconcentration of metal ions. Journal of Hazardous Materials, 172, 958-963.

Zhang, H. \& Shi, Y. (2010). Temperature-assisted ionic liquid dispersive liquid-liquid microextraction combined with high performance liquid chromatography for the determination of anthraquinones in Radix and Rhizoma Rhei samples. Talanta, 82, 1010-1016.

Zhang, W., Hu, Z., Liu, Y., Chen, H., Gao, S. \& Gaschnig, R. M. (2012). Total rock dissolution using ammonium bifluoride $\left(\mathrm{NH}_{4} \mathrm{HF}_{2}\right)$ in screw-top teflon vials: A new development in open-vessel digestion. Analytical Chemistry, 84, 10686-10693. 
Zhang, X, Oakes, K. D., Wang, S., Cui, S., Pawliszyn, J., Metcalfe, C. D. \& Servos, M. R. (2012). In vivo sampling of environmental organic contaminants in fish by solid-phase microextraction. TrAC Trends in Analytical Chemistry, 32, 31-39.

Zhao, Q. X., Chen, Y. W., Belzile, N. \& Wang, M. (2010). Low volume microwave digestion and direct determination of selenium in biological samples by hydride generation-atomic fluorescence spectrometry. Analytica Chimica Acta, 665, 123-128.

Zhou, C.-Y., Wong, M.-K. \& Koh, L.-L. (1996a). Microwave-assisted dilute acid extraction of trace metals from biological samples for atomic absorption spectrometric determination. Journal of Analytical Atomic Spectrometry, 11, 585-590.

Zhou, C.-Y., Wong, M.-K., Koh, L.-L. \& Wee, Y.-C. (1996b). Microwave digestion of biological samples with tetramethylammonium hydroxide and ethylenediaminetetraacetic acid for elemental determination. Talanta, 43, 1061-1068.

Zhou, Y., Zhang. C., Yan, Z., Li, K., Wang, L., Xie, Y., Li, F., Liu, Z. \& Yang, J. (2012). The use of copper (II) isonicotinate-based micro-solid-phase extraction for the analysis of polybrominated diphenyl ethers in soils. Analytica Chimica Acta, 747, 36-41.

Zhu, F., Xu, J., Ke, Y., Huang, S., Zeng, F., Luan, T. \& Ouyang, G. (2013). Applications of in vivo and in vitro solid-phase microextraction techniques in plant analysis: A review. Analytica Chimica Acta, 794, 1-14.

Zou, Z., Jang, A., MacKnight, E., Wu, P.-M., Do, J., Bishop, P. L. \& Ahn, C. H. (2008). Environmentally friendly disposable sensors with microfabricated on-chip planar bismuth electrode for in situ heavy metal ions measurement. Sensors and Actuators B, 134, 18-24. 